\title{
Finite dimensional simple modules of deformed current Lie algebras
}

\author{
Kentaro Wada
}

\begin{abstract}
The deformed current Lie algebra was introduced in [W] to study the representation theory of cyclotomic $q$-Schur algebras at $q=1$. In this paper, we classify finite dimensional simple modules of deformed current Lie algebras.
\end{abstract}

\section{Contents}

$\S 0$. Introduction 1

$\S$ 1. Deformed current Lie algebras $\mathfrak{s l}_{m}^{\langle\mathbf{Q}\rangle}[x]$ and $\mathfrak{g l}_{m}^{\langle\mathbf{Q}\rangle}[x]$

$\S$ 2. Representations of $\mathfrak{s l}_{m}^{\langle\mathbf{Q}\rangle}[x]$

$\S 3$. Representations of $\mathfrak{g l}_{m}^{\langle\mathbf{Q}\rangle}[x]$

$\S$ 4. Rank 1 case ; some relations in $U\left(\mathfrak{s l}_{2}^{\langle Q\rangle}[x]\right)$

$\S 5$. Rank 1 case ; finite dimensional simple modules of $U\left(\mathfrak{s l}_{2}^{\langle Q\rangle}[x]\right)$

$\S 6$. Finite dimensional simple $U\left(\mathfrak{s l}_{m}^{\langle\mathbf{Q}\rangle}[x]\right)$-modules

$\S 7$. Finite dimensional simple $U\left(\mathfrak{g} \mathfrak{l}_{m}^{\langle\mathbf{Q}\rangle}[x]\right)$-modules 36

Appendix A. Some combinatorics $\quad 40$

References 43

\section{$\S$ 0. INTRODUCTION}

0.1. The deformed current Lie algebra $\mathfrak{g}_{\widehat{\mathbf{Q}}}(\mathbf{m})$ was introduced in [W] to study the representation theory of cyclotomic $q$-Schur algebras at $q=1$. In this paper, we introduce the deformed current Lie algebra $\mathfrak{s l}_{m}^{\langle\mathbf{Q}\rangle}[x]$ and $\mathfrak{g l}_{m}^{\langle\mathbf{Q}\rangle}[x]$ over $\mathbb{C}$ associated with the special linear Lie algebra $\mathfrak{s l}_{m}$ and general linear Lie algebra $\mathfrak{g l}_{m}$ respectively. $\mathfrak{s l}_{m}^{\langle\mathbf{Q}\rangle}[x]$ (resp. $\mathfrak{g l}_{m}^{\langle\mathbf{Q}\rangle}[x]$ ) is a deformation of the current Lie algebra $\mathfrak{s l}_{m}[x]=\mathfrak{s l}_{m} \otimes_{\mathbb{C}} \mathbb{C}[x]$ (resp. $\mathfrak{g l}_{m}[x]=\mathfrak{g l}_{m} \otimes_{\mathbb{C}} \mathbb{C}[x]$ ) with deformation parameters $\mathbf{Q}=\left(Q_{1}, Q_{2}, \ldots, Q_{m-1}\right) \in \mathbb{C}^{m-1}$. Note that $\mathfrak{s l}_{m}^{\langle\mathbf{Q}\rangle}[x]\left(\right.$ resp. $\left.\mathfrak{g l}_{m}^{\langle\mathbf{Q}\rangle}[x]\right)$ is coincide with $\mathfrak{s l}_{m}[x]$ (resp. $\left.\mathfrak{g l}_{m}[x]\right)$ if $Q_{i}=0$ for all $i=1,2, \ldots, m-1$. The Lie algebra $\mathfrak{g}_{\widehat{\mathbf{Q}}}(\mathbf{m})$ introduced in $[\mathrm{W}]$ is isomorphic to $\mathfrak{g l}_{m}^{\langle\mathbf{Q}\rangle}[x]$ under a suitable choice of deformation parameters Q (Lemma 1.7).

0.2. The differences of the representation theory of $\mathfrak{s l}_{m}^{\langle\mathbf{Q}\rangle}[x]$ from one of $\mathfrak{s l}_{m}[x]$ appear in the following two points. The deformed current Lie algebra $\mathfrak{s l}_{m}^{\langle\mathbf{Q}\rangle}[x]$ has a family 
of 1-dimensional representations $\left\{\mathcal{L}^{\boldsymbol{\beta}} \mid \boldsymbol{\beta} \in \prod_{i=1}^{m-1} \mathbb{B}^{\left\langle Q_{i}\right\rangle}\right\}$, where

$$
\mathbb{B}^{\left\langle Q_{i}\right\rangle}= \begin{cases}\{0\} & \text { if } Q_{i}=0 \\ \mathbb{C} & \text { if } Q_{i} \neq 0\end{cases}
$$

although the 1-dimensional representation of $\mathfrak{s l}_{m}[x]$ is only the trivial representation (Lemma 6.2). (We remark that $\mathcal{L}^{(0, \ldots, 0)}$ is the trivial representation of $\mathfrak{s l}_{m}^{\langle\mathbf{Q}\rangle}[x]$.)

The second difference appears in the evaluation modules. For each $\gamma \in \mathbb{C}$, we can consider the evaluation homomorphism $\mathbf{e v}_{\gamma}: U\left(\mathfrak{s l}_{m}^{\langle\mathbf{Q}\rangle}[x]\right) \rightarrow U\left(\mathfrak{s l}_{m}\right)$ which is a deformation of the evaluation homomorphism for $\mathfrak{s l}_{m}[x]$ (see the paragraph 1.5 for the definition). Then we can consider the evaluation modules by regarding $U\left(\mathfrak{s l}_{m}\right)$ modules as $U\left(\mathfrak{s l}_{m}^{\langle\mathbf{Q}\rangle}[x]\right)$-modules through the evaluation homomorphism $\mathbf{e v}_{\gamma}$. The evaluation homomorphism $\mathbf{e v}_{\gamma}$ is surjective if $\gamma \neq Q_{i}^{-1}$ for all $i=1,2, \ldots, m-1$ such that $Q_{i} \neq 0$. However, $\mathbf{e v}_{\gamma}$ is not surjective if $\gamma=Q_{i}^{-1}$ for some $i=1,2, \ldots, m-1$. Moreover, in general, the evaluation module of a simple $U\left(\mathfrak{s l}_{m}\right)$-module at $\gamma \in \mathbb{C}$ is not simple if $\gamma=Q_{i}^{-1}$ for some $i=1,2, \ldots, m-1$ (see Remark 5.10).

0.3. It is a purpose of this paper to classify the finite dimensional simple modules of $\mathfrak{s l}_{m}^{\langle\mathbf{Q}\rangle}[x]$ and $\mathfrak{g l}_{m}^{\langle\mathbf{Q}\rangle}[x]$. A classification of the finite dimensional simple modules for the original current Lie algebra is well-known (e.g. [C], CP]). The classification for $\mathfrak{s l}_{m}^{\langle\mathbf{Q}\rangle}[x]$ (resp. $\left.\mathfrak{g l}_{m}^{\langle\mathbf{Q}\rangle}[x]\right)$ is an analogue of the original case.

Since $\mathfrak{s l}_{m}^{\langle\mathbf{Q}\rangle}[x]$ has the triangular decomposition (Proposition [1.4), we can develop the usual highest weight theory (see \$2). In particular, any finite dimensional simple $U\left(\mathfrak{s l}_{m}^{\langle\mathbf{Q}\rangle}[x]\right)$-module is isomorphic to a highest weight module $\mathcal{L}(\mathbf{u})$ of highest weight $\mathbf{u} \in \prod_{i=1}^{m-1} \prod_{t>0} \mathbb{C}$ (Proposition 2.6). Then it is enough to determine the highest weights such that the corresponding simple highest weight modules are finite dimensional. We obtain a classification of such highest weights as follows. Let $\mathbb{C}[x]_{\text {monic }}$ be the set of monic polynomials over $\mathbb{C}$ with the indeterminate variable $x$. For each $Q \in \mathbb{C}$, put

$$
\mathbb{C}[x]_{\text {monic }}^{\langle Q\rangle}= \begin{cases}\mathbb{C}[x]_{\text {monic }} & \text { if } Q=0, \\ \left\{\varphi \in \mathbb{C}[x]_{\text {monic }} \mid Q^{-1} \text { is not a root of } \varphi\right\} & \text { if } Q \neq 0\end{cases}
$$

We define the map $\prod_{i=1}^{m-1}\left(\mathbb{C}[x]_{\text {monic }}^{\left\langle Q_{i}\right\rangle} \times \mathbb{B}^{\left\langle Q_{i}\right\rangle}\right) \rightarrow \prod_{i=1}^{m-1} \prod_{t \geq 0} \mathbb{C}$,

$$
(\boldsymbol{\varphi}, \boldsymbol{\beta})=\left(\left(\varphi_{i}, \beta_{i}\right)\right)_{1 \leq i \leq m-1} \mapsto \mathbf{u}^{\langle\mathbf{Q}\rangle}(\boldsymbol{\varphi}, \boldsymbol{\beta})=\left(\mathbf{u}^{\langle\mathbf{Q}\rangle}(\boldsymbol{\varphi}, \boldsymbol{\beta})_{i, t}\right)_{1 \leq i \leq m-1, t \geq 0}
$$

by

$$
\mathbf{u}^{\langle\mathbf{Q}\rangle}(\boldsymbol{\varphi}, \boldsymbol{\beta})_{i, t}= \begin{cases}\gamma_{i, 1}^{t}+\gamma_{i, 2}^{t}+\cdots+\gamma_{i, n_{i}}^{t} & \text { if } Q_{i}=0 \\ \gamma_{i, 1}^{t}+\gamma_{i, 2}^{t}+\cdots+\gamma_{i, n_{i}}^{t}+Q_{i}^{-t} \beta_{i} & \text { if } Q_{i} \neq 0\end{cases}
$$


when $\varphi_{i}=\left(x-\gamma_{i, 1}\right)\left(x-\gamma_{i, 2}\right) \ldots\left(x-\gamma_{i, n_{i}}\right)(1 \leq i \leq m-1)$. Then we have the following classification of finite dimensional simple $U\left(\mathfrak{s l}_{m}^{\langle\mathbf{Q}\rangle}[x]\right)$-modules (Theorem 6.4).

Theorem: $\left\{\mathcal{L}\left(\mathbf{u}^{\langle\mathbf{Q}\rangle}(\boldsymbol{\varphi}, \boldsymbol{\beta})\right) \mid(\boldsymbol{\varphi}, \boldsymbol{\beta}) \in \prod_{i=1}^{m-1}\left(\mathbb{C}[x]_{\text {monic }}^{\left\langle Q_{i}\right\rangle} \times \mathbb{B}^{\left\langle Q_{i}\right\rangle}\right)\right\}$ gives a complete set of isomorphism classes of finite dimensional simple $U\left(\mathfrak{s l}_{m}^{\langle\mathbf{Q}\rangle}[x]\right)$ modules.

We remark that $\mathcal{L}\left(\mathbf{u}^{\langle\mathbf{Q}\rangle}(\boldsymbol{\varphi}, \boldsymbol{\beta})\right)$ is isomorphic to a subquotient of

$$
\left(\bigotimes_{j=1}^{m-1} \bigotimes_{k=1}^{n_{j}} L\left(\omega_{j}\right)^{\mathbf{e v}_{\gamma_{j}, k}}\right) \otimes \mathcal{L}^{\boldsymbol{\beta}},
$$

where $\left\{\omega_{j} \mid 1 \leq j \leq m-1\right\}$ is the set of fundamental weights for $\mathfrak{s l}_{m}, L\left(\omega_{j}\right)$ $(1 \leq j \leq m-1)$ is the simple highest weight $U\left(\mathfrak{s l}_{m}\right)$-module of highest weight $\omega_{j}$ and $L\left(\omega_{j}\right)^{\mathbf{e v}_{\gamma_{j}, k}}$ is the evaluation module of $L\left(\omega_{j}\right)$ at $\gamma_{j, k}$.

We also see that any finite dimensional simple $U\left(\mathfrak{g l}_{m}^{\langle\mathbf{Q}\rangle}[x]\right)$-module is isomorphic to a highest weight module $\mathcal{L}(\widetilde{\mathbf{u}})$ of highest weight $\widetilde{\mathbf{u}} \in \prod_{j=1}^{m} \prod_{t \geq 0} \mathbb{C}$ (Proposition 3.3). Note that $\mathfrak{s l}_{m}^{\langle\mathbf{Q}\rangle}[x]$ is a Lie subalgebra of $\mathfrak{g l}_{m}^{\langle\mathbf{Q}\rangle}[x]$ (Proposition 1.4 (iii)). The difference of representations of $\mathfrak{g l}_{m}^{\langle\mathbf{Q}\rangle}[x]$ from one of $\mathfrak{s}_{m}^{\langle\mathbf{Q}\rangle}[x]$ is given by the family of 1-dimensional $U\left(\mathfrak{g l}_{m}^{\langle\mathbf{Q}\rangle}[x]\right)$-modules $\left\{\widetilde{\mathcal{L}}^{\mathbf{h}} \mid \mathbf{h} \in \prod_{t \geq 0} \mathbb{C}\right\}$. We remark that $\widetilde{\mathcal{L}}^{\mathbf{h}}$ $\left(\mathbf{h} \in \prod_{t>0} \mathbb{C}\right)$ is isomorphic to the trivial representation $\mathcal{L}^{(0, \ldots, 0)}$ as a $U\left(\mathfrak{s l}_{m}^{\langle\mathbf{Q}\rangle}[x]\right)$ module when we restrict the action. We obtain the classification of finite dimensional simple $U\left(\mathfrak{g l}_{m}^{\langle\mathbf{Q}\rangle}[x]\right)$-modules as follows. We define the map $\prod_{i=1}^{m-1}\left(\mathbb{C}[x]_{\text {monic }}^{\left\langle Q_{i}\right\rangle} \times \mathbb{B}^{\left\langle Q_{i}\right\rangle}\right) \times$ $\prod_{t \geq 0} \mathbb{C} \rightarrow \prod_{j=1}^{m} \prod_{t \geq 0} \mathbb{C}$

$$
(\boldsymbol{\varphi}, \boldsymbol{\beta}, \mathbf{h})=\left(\left(\varphi_{i}, \beta_{i}\right)_{1 \leq i \leq m-1},\left(h_{t}\right)_{t \geq 0}\right) \mapsto \widetilde{\mathbf{u}}^{\langle\mathbf{Q}\rangle}(\boldsymbol{\varphi}, \boldsymbol{\beta}, \mathbf{h})=\left(\widetilde{\mathbf{u}}^{\langle\mathbf{Q}\rangle}(\boldsymbol{\varphi}, \boldsymbol{\beta}, \mathbf{h})_{j, t}\right)_{1 \leq j \leq m, t \geq 0}
$$

by

$$
\widetilde{\mathbf{u}}^{\langle\mathbf{Q}\rangle}(\boldsymbol{\varphi}, \boldsymbol{\beta}, \mathbf{h})_{j, t}= \begin{cases}\sum_{k=j}^{m-1} \mathbf{u}^{\langle\mathbf{Q}\rangle}(\boldsymbol{\varphi}, \boldsymbol{\beta})_{k, t}+h_{t} & \text { if } 1 \leq j \leq m-1 \text { and } t \geq 0, \\ h_{t} & \text { if } j=m \text { and } t \geq 0,\end{cases}
$$

where $\mathbf{u}^{\langle\mathbf{Q}\rangle}(\boldsymbol{\varphi}, \boldsymbol{\beta})_{k, t}$ is determined by (0.3.1). Then we have the following classification of finite dimensional simple $U\left(\mathfrak{g l}_{m}^{\langle\mathbf{Q}\rangle}[x]\right)$-modules (Theorem 7.4).

Theorem: $\left\{\mathcal{L}\left(\widetilde{\mathbf{u}}^{\langle\mathbf{Q}\rangle}(\boldsymbol{\varphi}, \boldsymbol{\beta}, \mathbf{h})\right) \mid(\boldsymbol{\varphi}, \boldsymbol{\beta}, \mathbf{h}) \in \prod_{i=1}^{m-1}\left(\mathbb{C}[x]_{\text {monic }}^{\left\langle Q_{i}\right\rangle} \times \mathbb{B}^{\left\langle Q_{i}\right\rangle}\right) \times \prod_{t \geq 0} \mathbb{C}\right\}$ gives a complete set of isomorphism classes of finite dimensional simple $U\left(\mathfrak{g} \mathfrak{l}_{m}^{\langle\mathbf{Q}\rangle}[x]\right)$-modules.

We remark that $\mathcal{L}\left(\widetilde{\mathbf{u}}^{\langle\mathbf{Q}\rangle}(\boldsymbol{\varphi}, \boldsymbol{\beta}, \mathbf{h})\right)$ is isomorphic to a subquotient of

$$
\left(\bigotimes_{j=1}^{m-1} \bigotimes_{k=1}^{n_{j}} L\left(\widetilde{\omega}_{j}\right)^{\widetilde{\mathbf{e v}}_{\gamma_{j}, k}}\right) \otimes \widetilde{\mathcal{L}}^{\boldsymbol{\beta}} \otimes \widetilde{\mathcal{L}}^{\mathbf{h}}
$$


(See 97 for definitions of $L\left(\widetilde{\omega}_{j}\right)^{\widetilde{\mathbf{e v}}_{\gamma_{j}, k}}, \widetilde{\mathcal{L}}^{\boldsymbol{\beta}}$ and $\widetilde{\mathcal{L}}^{\mathbf{h}}$.) We also remark that

$$
\begin{aligned}
& \mathcal{L}\left(\widetilde{\mathbf{u}}^{\langle\mathbf{Q}\rangle}(\boldsymbol{\varphi}, \boldsymbol{\beta}, \mathbf{h})\right) \cong \mathcal{L}\left(\mathbf{u}^{\langle\mathbf{Q}\rangle}(\boldsymbol{\varphi}, \boldsymbol{\beta})\right),
\end{aligned}
$$

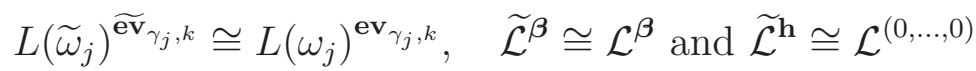

as $U\left(\mathfrak{s}_{m}^{\langle\mathbf{Q}\rangle}[x]\right)$-modules when we restrict the action.

Acknowledgements: This work was supported by JSPS KAKENHI Grant Number JP16K17565.

\section{$\S$ 1. Deformed CURREnt Lie Algebras $\mathfrak{s l}_{m}^{\langle\mathbf{Q}\rangle}[x]$ AND $\mathfrak{g l}_{m}^{\langle\mathbf{Q}\rangle}[x]$}

In this section, we give a definition of deformed current Lie algebras $\mathfrak{s l}_{m}^{\langle\mathbf{Q}\rangle}[x]$ and $\mathfrak{g l}_{m}^{\langle\mathbf{Q}\rangle}[x]$, and also give some basic facts. The definition of $\mathfrak{g l}_{m}^{\langle\mathbf{Q}\rangle}[x]$ in this section is different from one of $\mathfrak{g}_{\widehat{\mathbf{Q}}}(\mathbf{m})$ given in $[\mathbf{W}]$. The relation between $\mathfrak{g l}_{m}^{\langle\mathbf{Q}\rangle}[x]$ and $\mathfrak{g}_{\widehat{\mathbf{Q}}}(\mathbf{m})$ is given in Lemma 1.7 .

Definition 1.1. Put $\mathbf{Q}=\left(Q_{1}, Q_{2}, \ldots, Q_{m-1}\right) \in \mathbb{C}^{m-1}$. We define the Lie algebra $\mathfrak{s}_{m}^{\langle\mathbf{Q}\rangle}[x]$ over $\mathbb{C}$ by the following generators and defining relations:

Generators: $\mathcal{X}_{i, t}^{ \pm}, \mathcal{J}_{i, t}(1 \leq i \leq m-1, t \geq 0)$.

Relations:

$$
\begin{aligned}
& {\left[\mathcal{J}_{i, s}, \mathcal{J}_{j, t}\right]=0,} \\
& {\left[\mathcal{J}_{j, s}, \mathcal{X}_{i, t}^{ \pm}\right]= \pm a_{j i} \mathcal{X}_{i, s+t}^{ \pm},} \\
& {\left[\mathcal{X}_{i, t}^{+}, \mathcal{X}_{j, s}^{-}\right]=\delta_{i j}\left(\mathcal{J}_{i, s+t}-Q_{i} \mathcal{J}_{i, s+t+1}\right),} \\
& {\left[\mathcal{X}_{i, t}^{ \pm}, \mathcal{X}_{j, s}^{ \pm}\right]=0 \quad \text { if } j \neq i \pm 1,} \\
& {\left[\mathcal{X}_{i, t+1}^{+}, \mathcal{X}_{i \pm 1, s}^{+}\right]=\left[\mathcal{X}_{i, t}^{+}, \mathcal{X}_{i \pm 1, s+1}^{+}\right], \quad\left[\mathcal{X}_{i, t+1}^{-}, \mathcal{X}_{i \pm 1, s}^{-}\right]=\left[\mathcal{X}_{i, t}^{-}, \mathcal{X}_{i \pm 1, s+1}^{-}\right],} \\
& {\left[\mathcal{X}_{i, s}^{+},\left[\mathcal{X}_{i, t}^{+}, \mathcal{X}_{i \pm 1, u}^{+}\right]\right]=\left[\mathcal{X}_{i, s}^{-},\left[\mathcal{X}_{i, t}^{-}, \mathcal{X}_{i \pm 1, u}^{-}\right]\right]=0,} \\
& \text { where we put } a_{j i}= \begin{cases}2 & \text { if } j=i, \\
-1 & \text { if } j=i \pm 1, \\
0 & \text { otherwise. }\end{cases}
\end{aligned}
$$

We also define the Lie algebra $\mathfrak{g l}_{m}^{\langle\mathbf{Q}\rangle}[x]$ over $\mathbb{C}$ by the following generators and defining relations:

Generators: $\mathcal{X}_{i, t}^{ \pm}(1 \leq i \leq m-1, t \geq 0), \mathcal{I}_{j, t}(1 \leq j \leq m, t \geq 0)$.

Relations:

$$
\begin{aligned}
& {\left[\mathcal{I}_{i, s}, \mathcal{I}_{j, t}\right]=0} \\
& {\left[\mathcal{I}_{j, s}, \mathcal{X}_{i, t}^{ \pm}\right]= \pm a_{j i}^{\prime} \mathcal{X}_{i, s+t}^{ \pm}} \\
& {\left[\mathcal{X}_{i, t}^{+}, \mathcal{X}_{j, s}^{-}\right]=\delta_{i j}\left(\mathcal{J}_{i, s+t}-Q_{i} \mathcal{J}_{i, s+t+1}\right), \text { where we put } \mathcal{J}_{i, t}=\mathcal{I}_{i, t}-\mathcal{I}_{i+1, t}}
\end{aligned}
$$


together with the relations (L4)-(L6) in the above. In the relation (L'Q), we

$$
\text { put } a_{j i}^{\prime}= \begin{cases}1 & \text { if } j=i \\ -1 & \text { if } j=i+1 \\ 0 & \text { otherwise. }\end{cases}
$$

1.2. We call $\mathfrak{s l}_{m}^{\langle\mathbf{Q}\rangle}[x]$ (resp. $\mathfrak{g l}_{m}^{\langle\mathbf{Q}\rangle}[x]$ ) the deformed current Lie algebra associated with the special linear Lie algebra $\mathfrak{s l}_{m}$ (resp. the general linear Lie algebra $\mathfrak{g l}_{m}$ ). If $Q_{i}=0$ for all $i=1,2, \ldots, m-1$, then $\mathfrak{s l}_{m}^{\langle\mathbf{Q}\rangle}[x]$ (resp. $\left.\mathfrak{g l}_{m}^{\langle\mathbf{Q}\rangle}[x]\right)$ coincides with the current Lie algebra $\mathfrak{s l}_{m}[x]=\mathfrak{s l}_{m} \otimes_{\mathbb{C}} \mathbb{C}[x]$ (resp. $\mathfrak{g l}_{m}[x]=\mathfrak{g l}_{m} \otimes_{\mathbb{C}} \mathbb{C}[x]$ ) associated with $\mathfrak{s l}_{m}\left(\right.$ resp. $\left.\mathfrak{g l}_{m}\right)$. We can also regard $\mathfrak{s l}_{m}^{\langle\mathbf{Q}\rangle}[x]$ (resp. $\mathfrak{g l}_{m}^{\langle\mathbf{Q}\rangle}[x]$ ) as a filtered deformation of $\mathfrak{s l}_{m}[x]\left(\right.$ resp. $\left.\mathfrak{g l}_{m}[x]\right)$ in a similar way as in [W, Proposition 2.13].

1.3. For $1 \leq i \neq j \leq m$ and $t \geq 0$, we define an element $\mathcal{E}_{i, j ; t} \in \mathfrak{s l}_{m}^{\langle\mathbf{Q}\rangle}[x]$ (resp. $\left.\mathcal{E}_{i, j ; t} \in \mathfrak{g l}_{n}^{\langle\mathbf{Q}\rangle}[x]\right)$ by

$$
\mathcal{E}_{i, j ; t}= \begin{cases}{\left[\mathcal{X}_{i, 0}^{+},\left[\mathcal{X}_{i+1,0}^{+}, \ldots,\left[\mathcal{X}_{j-2,0}^{+}, \mathcal{X}_{j-1, t}^{+}\right] \ldots\right]\right]} & \text { if } j>i \\ {\left[\mathcal{X}_{i-1,0}^{-},\left[\mathcal{X}_{i-2,0}^{-}, \ldots,\left[\mathcal{X}_{j+1,0}^{-}, \mathcal{X}_{j, t}^{-}\right] \ldots\right]\right]} & \text { if } j<i\end{cases}
$$

In particular, we have $\mathcal{E}_{i, i+1 ; t}=\mathcal{X}_{i, t}^{+}$and $\mathcal{E}_{i+1, i ; t}=\mathcal{X}_{i, t}^{-}$.

Let $\mathbf{n}^{+}$and $\mathbf{n}^{-}$be the Lie subalgebra of $\mathfrak{s l}_{m}^{\langle\mathbf{Q}\rangle}[x]$ (also of $\mathfrak{g l}_{m}^{\langle\mathbf{Q}\rangle}[x]$ ) generated by

$$
\left\{\mathcal{X}_{i, t}^{+} \mid 1 \leq i \leq m-1, t \geq 0\right\} \text { and }\left\{\mathcal{X}_{i, t}^{-} \mid 1 \leq i \leq m-1, t \geq 0\right\}
$$

respectively. Let $\mathbf{n}^{0}$ (resp. $\quad \widetilde{\mathbf{n}}^{0}$ ) be the Lie subalgebra of $\mathfrak{s l}_{m}^{\langle\mathbf{Q}\rangle}[x]$ (resp. $\mathfrak{g l}_{m}^{\langle\mathbf{Q}\rangle}[x]$ ) generated by

$$
\left.\left\{\mathcal{J}_{i, t} \mid 1 \leq i \leq m-1, t \geq 0\right\} \quad \text { (resp. }\left\{\mathcal{I}_{i, t} \mid 1 \leq i \leq m, t \geq 0\right\}\right)
$$

By the relation (L1) (resp. (L'1)), we see that $\mathbf{n}^{0}$ (resp. $\widetilde{\mathbf{n}}^{0}$ ) is a commutative Lie subalgebra of $\mathfrak{s l}_{m}^{\langle\mathbf{Q}}[x]\left(\operatorname{resp} \mathfrak{g l}_{m}^{\langle\mathbf{Q}\rangle}[x]\right)$.

\section{Proposition 1.4.}

(i) $\left\{\mathcal{E}_{i, j ; t} \mid 1 \leq i \neq j \leq m, t \geq 0\right\} \cup\left\{\mathcal{J}_{i, t} \mid 1 \leq i \leq m-1, t \geq 0\right\}$ gives a basis of $\mathfrak{s l}_{m}^{\langle\mathbf{Q}\rangle}[x]$.

(ii) $\left\{\mathcal{E}_{i, j ; t} \mid 1 \leq i \neq j \leq m, t \geq 0\right\} \cup\left\{\mathcal{I}_{j, t} \mid 1 \leq j \leq m, t \geq 0\right\}$ gives a basis of $\mathfrak{g l}_{m}^{\langle\mathbf{Q}\rangle}[x]$.

(iii) There exists an injective homomorphism of Lie algebras

$$
\Upsilon: \mathfrak{s l}_{m}^{\langle\mathbf{Q}\rangle}[x] \rightarrow \mathfrak{g l}_{m}^{\langle\mathbf{Q}\rangle}[x] \text { such that } \mathcal{X}_{i, t}^{ \pm} \mapsto \mathcal{X}_{i, t}^{ \pm} \text {, and } \mathcal{J}_{i, t} \mapsto \mathcal{I}_{i, t}-\mathcal{I}_{i+1, t}
$$

(iv) We have the triangular decomposition

$$
\mathfrak{s}_{m}^{\langle\mathbf{Q}\rangle}[x]=\mathbf{n}^{-} \oplus \mathbf{n}^{0} \oplus \mathbf{n}^{+} \text {and } \mathfrak{g l}_{m}^{\langle\mathbf{Q}\rangle}[x]=\mathbf{n}^{-} \oplus \widetilde{\mathbf{n}}^{0} \oplus \mathbf{n}^{-} \quad \text { (as vector spaces) }
$$


In particular,

$$
\begin{aligned}
& \left\{\mathcal{E}_{i, j ; t} \mid 1 \leq i<j \leq m, t \geq 0\right\} \quad\left(\operatorname{resp} . \quad\left\{\mathcal{E}_{i, j ; t} \mid 1 \leq j<i \leq m, t \geq 0\right\}\right) \\
& \text { gives a basis of } \mathbf{n}^{+} \quad\left(\text { resp. } \mathbf{n}^{-}\right) \text {, and } \\
& \qquad\left\{\mathcal{J}_{i, t} \mid 1 \leq i \leq m-1, t \geq 0\right\} \quad\left(\operatorname{resp} . \quad\left\{\mathcal{I}_{j, t} \mid 1 \leq j \leq m, t \geq 0\right\}\right) \\
& \text { gives a basis of } \mathbf{n}^{0}\left(\text { resp. } \widetilde{\mathbf{n}}^{0}\right) .
\end{aligned}
$$

Proof. (i) and (ii) are proven in a similar way as in the proof of [W, Proposition 2.6]. By checking the defining relations, we see that $\Upsilon$ is well-defined. We also see that $\Upsilon$ is injective by investigating the basis given in (i) and (ii) under the homomorphism $\Upsilon$. Then we have (iii). (iv) folloes from (i) and (ii).

1.5. Evaluation homomorphisms and evaluation modules. The general linear Lie algebra $\mathfrak{g l}_{m}$ is a Lie algebra over $\mathbb{C}$ generated by $e_{i}, f_{i}(1 \leq i \leq m-1)$ and $K_{j}$ $(1 \leq j \leq m)$ together with the following defining relations:

$$
\begin{aligned}
& {\left[K_{i}, K_{j}\right]=0, \quad\left[K_{j}, e_{i}\right]=a_{j i}^{\prime} e_{i}, \quad\left[K_{j}, f_{i}\right]=-a_{j i}^{\prime} f_{i},} \\
& {\left[e_{i}, f_{j}\right]=\delta_{i j} H_{i}, \text { where } H_{i}=K_{i}-K_{i+1},} \\
& {\left[e_{i}, e_{j}\right]=\left[f_{i}, f_{j}\right]=0 \text { if } j \neq i \pm 1, \quad\left[e_{i},\left[e_{i}, e_{i \pm 1}\right]\right]=\left[f_{i},\left[f_{i}, f_{i \pm 1}\right]\right]=0 .}
\end{aligned}
$$

The special linear Lie algebra $\mathfrak{s l}_{m}$ is a Lie subalgebra of $\mathfrak{g l}_{m}$ generated by $e_{i}, f_{i}, H_{i}$ $(1 \leq i \leq m-1)$.

For each $\gamma \in \mathbb{C}$, by checking the defining relations, we have the homomorphisms of algebras (evaluation homomorphism)

$$
\mathbf{e v}_{\gamma}: U\left(\mathfrak{s l}_{m}^{\langle\mathbf{Q}\rangle}[x]\right) \rightarrow U\left(\mathfrak{s l}_{m}\right) \text { by } \mathcal{X}_{i, t}^{+} \mapsto\left(1-Q_{i} \gamma\right) \gamma^{t} e_{i}, \mathcal{X}_{i, t}^{-} \mapsto \gamma^{t} f_{i}, \mathcal{J}_{i, t} \mapsto \gamma^{t} H_{i}
$$

and

$$
\widetilde{\mathbf{e v}}_{\gamma}: U\left(\mathfrak{g l}_{m}^{\langle\mathbf{Q}\rangle}[x]\right) \rightarrow U\left(\mathfrak{g l}_{m}\right) \text { by } \mathcal{X}_{i, t}^{+} \mapsto\left(1-Q_{i} \gamma\right) \gamma^{t} e_{i}, \mathcal{X}_{i, t}^{-} \mapsto \gamma^{t} f_{i}, \mathcal{I}_{j, t} \mapsto \gamma^{t} K_{j}
$$

Clearly, the homomorphism $\mathbf{e v}_{\gamma}$ (resp. $\widetilde{\mathbf{e v}}_{\gamma}$ ) is surjective if $\gamma \neq Q_{i}^{-1}$ for all $i=$ $1, \ldots, m-1$ such that $Q_{i} \neq 0$.

For a $U\left(\mathfrak{s l}_{m}\right)$-module $M$ (resp. a $U\left(\mathfrak{g l}_{m}\right)$-module $\left.M\right)$, we can regard $M$ as a $U\left(\mathfrak{s l}_{m}^{\langle\mathbf{Q}\rangle}[x]\right)$-module (resp. a $U\left(\mathfrak{g l}_{m}^{\langle\mathbf{Q}\rangle}[x]\right)$-module) through the evaluation homomorphism $\mathbf{e v}_{\gamma}$ (resp. $\widetilde{\mathbf{e v}}_{\gamma}$ ). We call it the evaluation module, and denote it by $M^{\mathbf{e}} \mathbf{v}_{\gamma}$

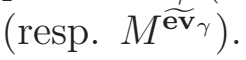

1.6. In the rest of this section, we give a relation with the Lie algebra $\mathfrak{g}_{\widehat{\mathbf{Q}}}(\mathbf{m})$ introduced in [W, Definition 2.2].

Let $\mathbf{m}=\left(m_{1}, \ldots, m_{r}\right)$ be an $r$-tuple of positive integers such that $\sum_{k=1}^{r} m_{k}=m$. Put $\Gamma(\mathbf{m})=\left\{(i, k) \mid 1 \leq i \leq m_{k}, 1 \leq k \leq r\right\}$ and $\Gamma^{\prime}(\mathbf{m})=\Gamma(\mathbf{m}) \backslash\left\{\left(m_{r}, r\right)\right\}$. Then 
we have the bijective map

$$
\zeta: \Gamma(\mathbf{m}) \rightarrow\{1,2, \ldots, m\} \text { such that }(i, k) \mapsto \sum_{j=1}^{k-1} m_{j}+i
$$

For $(i, k) \in \Gamma(\mathbf{m})$ and $j \in \mathbb{Z}$ such that $1 \leq \zeta((i, k))+j \leq m$, put $(i+j, k)=$ $\zeta^{-1}(\zeta((i, k))+j)$. For $(i, k) \in \Gamma^{\prime}(\mathbf{m})$ and $(j, l) \in \Gamma(\mathbf{m})$, put $a_{(j, l)(i, k)}^{\prime}=a_{\zeta((j, l)) \zeta((i, k))}^{\prime}$. Take $\widehat{\mathbf{Q}}=\left(\widehat{Q}_{1}, \ldots, \widehat{Q}_{r-1}\right) \in \mathbb{C}^{r-1}$. Then the Lie algebra $\mathfrak{g}_{\widehat{\mathbf{Q}}}(\mathbf{m})$ in $[\mathrm{W}$, Definition $2.2]$ is defined by the generators $\mathcal{X}_{(i, k), t}^{ \pm}, \mathcal{I}_{(j, l), t}\left((i, k) \in \Gamma^{\prime}(\mathbf{m}),(j, l) \in \Gamma(\mathbf{m}), t \geq 0\right)$ together with the following defining relations:

$$
\begin{aligned}
& {\left[\mathcal{I}_{(i, k), s}, \mathcal{I}_{(j, l), t}\right]=0, \quad\left[\mathcal{I}_{(j, l), s}, \mathcal{X}_{(i, k), t}^{ \pm}\right]= \pm a_{(j, l)(i, k)}^{\prime} \mathcal{X}_{(i, k), s+t}^{ \pm},} \\
& {\left[\mathcal{X}_{(i, k), t}^{+}, \mathcal{X}_{(j, l), s}^{-}\right]=\delta_{(i, k)(j, l)} \begin{cases}\mathcal{J}_{(i, k), s+t} & \text { if } i \neq m_{k}, \\
-\widehat{Q}_{k} \mathcal{J}_{\left(m_{k}, k\right), s+t}+\mathcal{J}_{\left(m_{k}, k\right), s+t+1} & \text { if } i=m_{k},\end{cases} } \\
& {\left[\mathcal{X}_{(i, k), t}^{ \pm}, \mathcal{X}_{(j, l), s}^{ \pm}\right]=0 \quad \text { if }(j, l) \neq(i \pm 1, k),} \\
& {\left[\mathcal{X}_{(i, k), t+1}^{+}, \mathcal{X}_{(i \pm 1, k), s}^{+}\right]=\left[\mathcal{X}_{(i, k), t}^{+}, \mathcal{X}_{(i \pm 1, k), s+1}^{+}\right], \quad\left[\mathcal{X}_{(i, k), t+1}^{-}, \mathcal{X}_{(i \pm 1, k), s}^{-}\right]=\left[\mathcal{X}_{(i, k), t}^{-}, \mathcal{X}_{(i \pm 1, k), s+1}^{-}\right],} \\
& {\left[\mathcal{X}_{(i, k), s}^{+},\left[\mathcal{X}_{(i, k), t}^{+}, \mathcal{X}_{(i \pm 1, k), u}^{+}\right]\right]=\left[\mathcal{X}_{(i, k), s}^{-},\left[\mathcal{X}_{(i, k), t}^{-}, \mathcal{X}_{(i \pm 1, k), u}^{-}\right]\right]=0,}
\end{aligned}
$$

where we put $\mathcal{J}_{(i, k), t}=\mathcal{I}_{(i, k), t}-\mathcal{I}_{(i+1, k), t}$. Then we have the following isomorphism between $\mathfrak{g l}_{m}^{\langle\mathbf{Q}\rangle}[x]$ and $\mathfrak{g}_{\widehat{\mathbf{Q}}}(\mathbf{m})$ under the suitable choice of the deformation parameters Q.

Lemma 1.7. Assume that $\widehat{Q}_{i} \neq 0$ for all $i=1,2, \ldots, r-1$. We take $\mathbf{Q}=$ $\left(Q_{1}, Q_{2}, \ldots, Q_{m-1}\right) \in \mathbb{C}^{m-1}$ as

$$
Q_{i}= \begin{cases}\widehat{Q}_{k}^{-1} & \text { if } \zeta^{-1}(i)=\left(m_{k}, k\right) \text { for some } k=1,2, \ldots, r-1 \\ 0 & \text { otherwise. }\end{cases}
$$

Then we have the isomorphism of Lie algebras $\Phi: \mathfrak{g l}_{m}^{\langle\mathbf{Q}\rangle}[x] \rightarrow \mathfrak{g}_{\widehat{\mathbf{Q}}}(\mathbf{m})$ such taht

$$
\begin{aligned}
& \mathcal{X}_{i, t}^{+} \mapsto \begin{cases}\mathcal{X}_{\zeta^{-1}(i), t}^{+} & \text {if } \zeta^{-1}(i) \neq\left(m_{k}, k\right) \text { for all } k=1, \ldots, r-1, \\
-\widehat{Q}_{k}^{-1} \mathcal{X}_{\zeta^{-1}(i), t}^{+} & \text {if } \zeta^{-1}(i)=\left(m_{k}, k\right) \text { for some } k=1, \ldots, r-1,\end{cases} \\
& \mathcal{X}_{i, t}^{-} \mapsto \mathcal{X}_{\zeta^{-1}(i), t}^{-}, \quad \mathcal{I}_{j, t} \mapsto \mathcal{I}_{\zeta^{-1}(j), t} .
\end{aligned}
$$

Proof. We see the well-definedness of $\Phi$ by checking the defining relations. The inverse homomorphism of $\Phi$ is given by

$$
\mathcal{X}_{(i, k), t}^{+} \mapsto\left\{\begin{array}{ll}
\mathcal{X}_{\zeta((i, k)), t}^{+} & \text {if } i \neq m_{k}, \\
-\widehat{Q}_{k} \mathcal{X}_{\zeta((i, k)), t}^{+} & \text {if } i=m_{k},
\end{array} \quad \mathcal{X}_{(i, k), t}^{-} \mapsto \mathcal{X}_{\zeta((i, k)), t}^{-}, \quad \mathcal{I}_{(j, l), t} \mapsto \mathcal{I}_{\zeta((j, l)), t} .\right.
$$




\section{$\S$ 2. Representations of $\mathfrak{s i}_{m}^{\langle\mathbf{Q}\rangle}[x]$}

In this section, we give some fundamental results for finite dimensional $U\left(\mathfrak{s l}_{m}^{\langle\mathbf{Q}\rangle}[x]\right)$ modules by using the standard argument.

2.1. Put $\mathfrak{h}=\bigoplus_{i=1}^{m-1} \mathbb{C} \mathcal{J}_{i, 0} \subset \mathfrak{s l}_{m}^{\langle\mathbf{Q}\rangle}[x]$, then $\mathfrak{h}$ is a commutative Lie subalgebra of $\mathfrak{s}_{m}^{\langle\mathbf{Q}\rangle}[x]$. (Note that, if $Q_{i}=0$ for all $i=1, \ldots, m-1, \mathfrak{h}$ is a Cartan subalgebra of $\mathfrak{s l}_{m}$.) Let $\mathfrak{h}^{*}$ be the dual space of $\mathfrak{h}$. For each $i=1,2, \ldots, m-1$, we take $\alpha_{i} \in \mathfrak{h}^{*}$ as $\alpha_{i}\left(\mathcal{J}_{j, 0}\right)=a_{j i}$ for $j=1, \ldots, m-1$. Put $Q^{+}=\bigoplus_{i=1}^{m-1} \mathbb{Z}_{\geq 0} \alpha_{i} \subset \mathfrak{h}^{*}$. We define the partial order on $\mathfrak{h}^{*}$ by $\lambda \geq \mu$ if $\lambda-\mu \in Q^{+}$for $\lambda, \mu \in \mathfrak{h}$.

2.2. For $U\left(\mathfrak{s l}_{m}^{\langle\mathbf{Q}\rangle}[x]\right)$-mdoule $M$, we consider the decomposition $M=\bigoplus_{\lambda \in \mathfrak{h}^{*}} \widetilde{M}_{\lambda}$, where $\widetilde{M}_{\lambda}=\left\{x \in M \mid(h-\lambda(h))^{N} \cdot x=0\right.$ for $h \in \mathfrak{h}$ and $\left.N \gg 0\right\}$, namely $M=$ $\bigoplus_{\lambda \in \mathfrak{h}^{*}} \widetilde{M}_{\lambda}$ is the decomposition to the generalized simultaneous eigenspaces for the action of $\mathfrak{h}$. By the relation (L2), we have

$$
\mathcal{X}_{i, t}^{ \pm} \cdot \widetilde{M}_{\lambda} \subset \widetilde{M}_{\lambda \pm \alpha_{i}} \quad(1 \leq i \leq m-1, t \geq 0)
$$

Thus, if $U\left(\mathfrak{s l}_{m}^{\langle\mathbf{Q}\rangle}[x]\right)$-module $M \neq 0$ is finite dimensional, there exists $\lambda \in \mathfrak{h}^{*}$ such that $\widetilde{M}_{\lambda} \neq 0$ and $\mathcal{X}_{i, t}^{+} \cdot \widetilde{M}_{\lambda}=0$ for all $i=1,2, \ldots, m-1$ and $t \geq 0$. On the other hand, $\widetilde{M}_{\lambda}\left(\lambda \in \mathfrak{h}^{*}\right)$ is closed under the action of $\mathbf{n}^{0}$ by the relation (L1). Thus, we can take a simultaneous eigenvector $v \in \widetilde{M}_{\lambda}$ for the action of $\mathbf{n}^{0}$. Then we have the following lemma.

Lemma 2.3. For a finite dimensional $U\left(\mathfrak{s l}_{m}^{\langle\mathbf{Q}\rangle}[x]\right)$-module $M \neq 0$, there exists $v_{0} \in$ $M\left(v_{0} \neq 0\right)$ satisfying the following conditions:

(i) $\mathcal{X}_{i, t}^{+} \cdot v_{0}=0$ for all $i=1, \ldots, m-1$ and $t \geq 0$,

(ii) $\mathcal{J}_{i, t} \cdot v_{0}=u_{i, t} v_{0}\left(u_{i, t} \in \mathbb{C}\right)$ for each $i=1, \ldots, m-1$ and $t \geq 0$.

Moreover, if $M$ is simple, we have $M=U\left(\mathfrak{s l}_{m}^{\langle\mathbf{Q}\rangle}[x]\right) \cdot v_{0}$.

2.4. Highest weight modules. For $U\left(\mathfrak{s l}_{m}^{\langle\mathbf{Q}\rangle}[x]\right)$-module $M$, we say that $M$ is a highest weight module if there exists $v_{0} \in M$ satisfying the following conditions:

(i) $M$ is generated by $v_{0}$ as a $U\left(\mathfrak{s l}_{m}^{\langle\mathbf{Q}\rangle}[x]\right)$-module.

(ii) $\mathcal{X}_{i, t}^{+} \cdot v_{0}=0$ for all $i=1, \ldots, m-1$ and $t \geq 0$.

(iii) $\mathcal{J}_{i, t} \cdot v_{0}=u_{i, t} v_{0}\left(u_{i, t} \in \mathbb{C}\right)$ for each $i=1, \ldots, m-1$ and $t \geq 0$.

In this case, we say that $\left(u_{i, t}\right)_{1 \leq i \leq m-1, t \geq 0} \in \prod_{i=1}^{m-1} \prod_{t \geq 0} \mathbb{C}$ is the highest weight of $M$, and that $v_{0}$ is a highest weight vector of $M$.

Let $M$ be a highest weight $U\left(\mathfrak{s l}_{m}^{\langle\mathbf{Q}\rangle}[x]\right)$-module with a highest weight $\mathbf{u}=$ $\left(u_{i, t}\right)_{1 \leq i \leq m-1, t \geq 0} \in \prod_{i=1}^{m-1} \prod_{t \geq 0} \mathbb{C}$ and a highest weight vector $v_{0} \in M$. Thanks to the triangular decomposition (Proposition 1.4 (iv)) together with the above conditions, we have $M=U\left(\mathbf{n}^{-}\right) \cdot v_{0}$. Let $\lambda_{\mathbf{u}} \in \mathfrak{h}^{*}$ be as $\lambda_{\mathbf{u}}\left(\mathcal{J}_{i, 0}\right)=u_{i, 0}$ for $i=1, \ldots, m-1$. By $M=U\left(\mathbf{n}^{-}\right) \cdot v_{0}$ and the relation (L2), we have the weight space decomposition

$$
M=\bigoplus_{\substack{\mu \in \mathfrak{h}^{*} \\ \mu \leq \lambda_{\mathbf{u}}}} M_{\mu}, \text { where } M_{\mu}=\{x \in M \mid h \cdot x=\mu(h) \cdot x \text { for } h \in \mathfrak{h}\},
$$


and we also have $\operatorname{dim}_{\mathbb{C}} M_{\lambda_{\mathbf{u}}}=1$.

2.5. Verma modules. For $\mathbf{u}=\left(u_{i, t}\right) \in \prod_{i=1}^{m-1} \prod_{t \geq 0} \mathbb{C}$, let $\mathfrak{I}(\mathbf{u})$ be the left ideal of $U\left(\mathfrak{s l}_{m}^{\langle\mathbf{Q}\rangle}[x]\right)$ generated by $\mathcal{X}_{i, t}^{+}(1 \leq i \leq m-1, t \geq 0)$ and $\mathcal{J}_{i, t}-u_{i, t}(1 \leq i \leq m-1$, $t \geq 0)$. We define the Verma module $\left.\mathcal{M}(\mathbf{u})=U(\mathfrak{s}\}_{m}^{\langle\mathbf{Q}\rangle}[x]\right) / \mathfrak{I}(\mathbf{u})$. Then $\mathcal{M}(\mathbf{u})$ is a highest weight module of highest weight $\mathbf{u}$, and any highest weight module of highest weight $\mathbf{u}$ is realized as a quotient of the Verma module $\mathcal{M}(\mathbf{u})$. By the weight space decomposition (2.4.1), we see that $\mathcal{M}(\mathbf{u})$ has the unique maximal proper submodule $\operatorname{rad} \mathcal{M}(\mathbf{u})$. Put $\mathcal{L}(\mathbf{u})=\mathcal{M}(\mathbf{u}) / \operatorname{rad} \mathcal{M}(\mathbf{u})$, then we have the following proposition.

Proposition 2.6. For $\mathbf{u}=\left(u_{i, t}\right) \in \prod_{i=1}^{m-1} \prod_{t>0} \mathbb{C}$, a highest weight simple $U\left(\mathfrak{s l}_{m}^{\langle\mathbf{Q}\rangle}[x]\right)$ module of highest weight $\mathbf{u}$ is isomorphic to $\overline{\mathcal{L}}(\mathbf{u})$. Moreover, any finite dimensional simple $U\left(\mathfrak{s}_{m}^{\langle\mathbf{Q}\rangle}[x]\right)$-module is isomorphic to $\mathcal{L}(\mathbf{u})$ for some $\mathbf{u}=\left(u_{i, t}\right) \in \prod_{i=1}^{m-1} \prod_{t \geq 0} \mathbb{C}$.

Proof. By Lemma 2.3, a finite dimensional simple $U\left(\mathfrak{s l}_{m}^{\langle\mathbf{Q}\rangle}[x]\right)$-module is a highest weight module. Then we have the proposition by the above arguments.

\section{$\S$ 3. Representations of $\mathfrak{g l}_{m}^{\langle\mathbf{Q}\rangle}[x]$}

For finite dimensional $U\left(\mathfrak{g l}_{m}^{\langle\mathbf{Q}\rangle}[x]\right)$-modules, we can develop a similar argument as in the case of $U\left(\mathfrak{s l}_{m}^{\langle\mathbf{Q}\rangle}[x]\right)$ discussed in the previous section. In this section, we give only some notation for $U\left(\mathfrak{g l}_{m}^{\langle\mathbf{Q}\rangle}[x]\right)$-modules.

3.1. Highest weight modules. For $U\left(\mathfrak{g l}_{m}^{\langle\mathbf{Q}\rangle}[x]\right)$-module $M$, we say that $M$ is a highest weight module if there exists $v_{0} \in M$ satisfying the following conditions:

(i) $M$ is generated by $v_{0}$ as a $U\left(\mathfrak{g l}_{m}^{\langle\mathbf{Q}\rangle}[x]\right)$-module.

(ii) $\mathcal{X}_{i, t}^{+} \cdot v_{0}=0$ for all $i=1, \ldots, m-1$ and $t \geq 0$.

(iii) $\mathcal{I}_{j, t} \cdot v_{0}=\widetilde{u}_{j, t} v_{0}\left(\widetilde{u}_{j, t} \in \mathbb{C}\right)$ for each $j=1, \ldots, m$ and $t \geq 0$.

In this case, we say that $\left(\widetilde{u}_{j, t}\right)_{1 \leq j \leq m, t \geq 0} \in \prod_{j=1}^{m} \prod_{t \geq 0} \mathbb{C}$ is the highest weight of $M$, and that $v_{0}$ is a highest weight vector of $M$.

3.2. Verma modules. For $\widetilde{\mathbf{u}}=\left(\widetilde{u}_{j, t}\right) \in \prod_{j=1}^{m} \prod_{t \geq 0} \mathbb{C}$, let $\mathfrak{I}(\widetilde{\mathbf{u}})$ be the left ideal of $U\left(\mathfrak{g l}_{m}^{\langle\mathbf{Q}\rangle}[x]\right)$ generated by $\mathcal{X}_{i, t}^{+}(1 \leq i \leq m-1, t \geq 0)$ and $\mathcal{I}_{j, t}-\widetilde{u}_{j, t}(1 \leq j \leq m$, $t \geq 0)$. We define the Verma module $\mathcal{M}(\widetilde{\mathbf{u}})=U\left(\mathfrak{g l}_{m}^{\langle\mathbf{Q}\rangle}[x]\right) / \mathfrak{I}(\widetilde{\mathbf{u}})$. Then $\mathcal{M}(\widetilde{\mathbf{u}})$ is a highest weight module of highest weight $\widetilde{\mathbf{u}}$, and any highest weight module of highest weight $\widetilde{\mathbf{u}}$ is realized as a quotient of the Verma module $\mathcal{M}(\widetilde{\mathbf{u}}) . \mathcal{M}(\widetilde{\mathbf{u}})$ has the unique maximal proper submodule $\operatorname{rad} \mathcal{M}(\widetilde{\mathbf{u}}) . \operatorname{Put} \mathcal{L}(\widetilde{\mathbf{u}})=\mathcal{M}(\widetilde{\mathbf{u}}) / \operatorname{rad} \mathcal{M}(\widetilde{\mathbf{u}})$, then we have the following proposition.

Proposition 3.3. For $\widetilde{\mathbf{u}}=\left(\widetilde{u}_{j, t}\right) \in \prod_{j=1}^{m} \prod_{t>0} \mathbb{C}$, a highest weight simple $U\left(\mathfrak{g} \mathfrak{g}_{m}^{\langle\mathbf{Q}\rangle}[x]\right)$ module of highest weight $\widetilde{\mathbf{u}}$ is isomorphic to $\mathcal{L}(\widetilde{\mathbf{u}})$. Moreover, any finite dimensional simple $U\left(\mathfrak{g l}_{m}^{\langle\mathbf{Q}\rangle}[x]\right)$-module is isomorphic to $\mathcal{L}(\widetilde{\mathbf{u}})$ for some $\widetilde{\mathbf{u}}=\left(u_{j, t}\right) \in \prod_{j=1}^{m} \prod_{t \geq 0} \mathbb{C}$. 


\section{$\S$ 4. RANK 1 CASE ; SOME RELATIONS IN $U\left(\mathfrak{s l}_{2}^{\langle Q\rangle}[x]\right)$}

4.1. Take $Q \in \mathbb{C}$, then $\mathfrak{s l}_{2}^{\langle Q\rangle}[x]$ is a Lie algebra over $\mathbb{C}$ generated by $\mathcal{X}_{t}^{ \pm}$and $\mathcal{J}_{t}$ $\left(t \in \mathbb{Z}_{\geq 0}\right)$ together with the following defining relations:

$$
\begin{aligned}
& {\left[\mathcal{J}_{s}, \mathcal{J}_{t}\right]=0} \\
& {\left[\mathcal{J}_{s}, \mathcal{X}_{t}^{ \pm}\right]= \pm 2 \mathcal{X}_{s+t}^{ \pm}} \\
& {\left[\mathcal{X}_{t}^{+}, \mathcal{X}_{s}^{-}\right]=\mathcal{J}_{s+t}-Q \mathcal{J}_{s+t+1}} \\
& {\left[\mathcal{X}_{t}^{ \pm}, \mathcal{X}_{s}^{ \pm}\right]=0}
\end{aligned}
$$

(In the rank 1 case, we omit the first index of the generators since it is trivial.) By checking the defining relations, we see that there exists the algebra antiautomorphism $\dagger: U\left(\mathfrak{s l}_{2}^{\langle Q\rangle}[x]\right) \rightarrow U\left(\mathfrak{s}_{2}^{\langle Q\rangle}[x]\right)$ such that

$$
\dagger\left(\mathcal{X}_{t}^{+}\right)=\mathcal{X}_{t}^{-}, \quad \dagger\left(\mathcal{X}_{t}^{-}\right)=\mathcal{X}_{t}^{+}, \quad \dagger\left(\mathcal{J}_{t}\right)=\mathcal{J}_{t}
$$

Clearly, $\dagger^{2}$ is the identity on $U\left(\mathfrak{s l}_{2}^{\langle Q\rangle}[x]\right)$.

4.2. For $t, b \in \mathbb{Z}_{\geq 0}$, we define an element $\mathcal{X}_{t}^{+(b)}$ (resp. $\left.\mathcal{X}_{t}^{-(b)}\right)$ of $U\left(\mathfrak{s l}_{2}^{\langle Q\rangle}[x]\right)$ by

$$
\mathcal{X}_{t}^{ \pm(b)}=\frac{\left(\mathcal{X}_{t}^{ \pm}\right)^{b}}{b !}
$$

For convenience, we put $\mathcal{X}_{t}^{ \pm(b)}=0$ for $b \in \mathbb{Z}_{<0}$. by

For $t, p, h \in \mathbb{Z}_{\geq 0}$, we define an element $\mathcal{X}_{t}^{+((p) ; h)}\left(\right.$ resp. $\left.\mathcal{X}_{t}^{-((p) ; h)}\right)$ of $U\left(\mathfrak{s l}_{2}^{\langle Q\rangle}[x]\right)$

$$
\mathcal{X}_{t}^{ \pm((0) ; h)}=1, \quad \mathcal{X}_{t}^{ \pm((p) ; h)}=\sum_{w=0}^{p}\left(\begin{array}{c}
p \\
w
\end{array}\right)(-Q)^{w} \mathcal{X}_{t+p h+w}^{ \pm} \quad \text { for } p>0
$$

Clearly, we have $\dagger\left(\mathcal{X}_{t}^{+((p) ; h)}\right)=\mathcal{X}_{t}^{-((p) ; h)}$. For examples, we have

$$
\begin{aligned}
& \mathcal{X}_{t}^{ \pm((0) ; h)}=1, \quad \mathcal{X}_{t}^{ \pm((1) ; h)}=\mathcal{X}_{t+h}^{ \pm}+(-Q) \mathcal{X}_{t+h+1}^{ \pm}, \\
& \mathcal{X}_{t}^{ \pm((2) ; h)}=\mathcal{X}_{t+2 h}^{ \pm}+2(-Q) \mathcal{X}_{t+2 h+1}^{ \pm}+(-Q)^{2} \mathcal{X}_{t+2 h+2}^{ \pm}, \\
& \mathcal{X}_{t}^{ \pm((3) ; h)}=\mathcal{X}_{t+3 h}^{ \pm}+3(-Q) \mathcal{X}_{t+3 h+1}^{ \pm}+3(-Q)^{2} \mathcal{X}_{t+3 h+2}^{ \pm}+(-Q)^{3} \mathcal{X}_{t+3 h+3}^{ \pm} .
\end{aligned}
$$

For $s, p \in \mathbb{Z}_{\geq 0}$, we define an element $\mathcal{J}_{s}^{\langle p\rangle}$ of $\left.U\left(\mathfrak{s l}_{2}^{\langle Q\rangle}[x]\right)\right)$ inductively on $p$ by

$$
\mathcal{J}_{s}^{\langle 0\rangle}=1, \quad \mathcal{J}_{s}^{\langle p\rangle}=\frac{1}{p} \sum_{z=1}^{p}(-1)^{z-1}\left(\sum_{w=0}^{z}\left(\begin{array}{c}
z \\
w
\end{array}\right)(-Q)^{w} \mathcal{J}_{z s+w}\right) \mathcal{J}_{s}^{\langle p-z\rangle} \text { for } p>0
$$


For examples, we have

$$
\begin{aligned}
& \mathcal{J}_{s}^{\langle 0\rangle}= 1, \quad \mathcal{J}_{s}^{\langle 1\rangle}=\mathcal{J}_{s}+(-Q) \mathcal{J}_{s+1} \\
& \mathcal{J}_{s}^{\langle 2\rangle}= \frac{1}{2}\left(\left(\mathcal{J}_{s}^{2}-\mathcal{J}_{2 s}\right)+2(-Q)\left(\mathcal{J}_{s} \mathcal{J}_{s+1}-\mathcal{J}_{2 s+1}\right)+(-Q)^{2}\left(\mathcal{J}_{s+1}^{2}-\mathcal{J}_{2 s+2}\right)\right) \\
& \mathcal{J}_{s}^{\langle 3\rangle}=\frac{1}{3}\left(\left(\mathcal{J}_{s}^{3}-2 \mathcal{J}_{s} \mathcal{J}_{2 s}+\mathcal{J}_{3 s}\right)+3(-Q)\left(\mathcal{J}_{s}^{2} \mathcal{J}_{s+1}-\mathcal{J}_{s} \mathcal{J}_{2 s+1}-\mathcal{J}_{s+1} \mathcal{J}_{2 s}+\mathcal{J}_{3 s+1}\right)\right. \\
& \quad+(-Q)^{2}\left(3 \mathcal{J}_{s} \mathcal{J}_{s+1}^{2}-2 \mathcal{J}_{s} \mathcal{J}_{2 s+2}-4 \mathcal{J}_{s+1} \mathcal{J}_{2 s+1}+3 \mathcal{J}_{3 s+2}\right) \\
&\left.\quad+(-Q)^{3}\left(\mathcal{J}_{s+1}^{3}-2 \mathcal{J}_{s+1} \mathcal{J}_{2 s+2}+\mathcal{J}_{3 s+3}\right)\right)
\end{aligned}
$$

Lemma 4.3. For $s, t, p \in \mathbb{Z}_{\geq 0}$, we have the following relations in $U\left(\mathfrak{s i}_{2}^{\langle Q\rangle}[x]\right)$.

(i) $\left[\mathcal{J}_{s}^{\langle p\rangle}, \mathcal{X}_{t}^{+}\right]=\sum_{z=1}^{p}(-1)^{z+1}(z+1) \mathcal{J}_{s}^{\langle p-z\rangle} \mathcal{X}_{t}^{+((z) ; s)}$.
(ii) $\left[\mathcal{J}_{s}^{\langle p\rangle}, \mathcal{X}_{t}^{-}\right]=-\sum_{z=1}^{p}(-1)^{z+1}(z+1) \mathcal{X}_{t}^{-((z) ; s)} \mathcal{J}_{s}^{\langle p-z\rangle}$.

Proof. (ii) follows from (i) by applying the algebra anti-automorphism $\dagger$ defined in (4.1.1). Then, we prove only (i) by the induction on $p$.

If $p=0,(\mathrm{i})$ is clear. If $p>0$, by the definition (4.2.2), we have

$$
\mathcal{J}_{s}^{\langle p\rangle} \mathcal{X}_{t}^{+}=\frac{1}{p} \sum_{z=1}^{p}(-1)^{z-1}\left(\sum_{w=0}^{z}\left(\begin{array}{c}
z \\
w
\end{array}\right)(-Q)^{w} \mathcal{J}_{z s+w}\right) \mathcal{J}_{s}^{\langle p-z\rangle} \mathcal{X}_{t}^{+}
$$

By the assumption of the induction, we have

$$
\begin{aligned}
\mathcal{J}_{s}^{\langle p\rangle} \mathcal{X}_{t}^{+}= & \frac{1}{p} \sum_{z=1}^{p}(-1)^{z-1}\left(\sum_{w=0}^{z}\left(\begin{array}{c}
z \\
w
\end{array}\right)(-Q)^{w} \mathcal{J}_{z s+w}\right) \\
& \times\left(\mathcal{X}_{t}^{+} \mathcal{J}_{s}^{\langle p-z\rangle}+\sum_{k=1}^{p-z}(-1)^{k+1}(k+1) \mathcal{J}_{s}^{\langle p-z-k\rangle} \mathcal{X}_{t}^{+((k) ; s)}\right) \\
= & \frac{1}{p} \sum_{z=1}^{p}(-1)^{z-1} \sum_{w=0}^{z}\left(\begin{array}{c}
z \\
w
\end{array}\right)(-Q)^{w}\left(\mathcal{X}_{t}^{+} \mathcal{J}_{z s+w}+2 \mathcal{X}_{t+z s+w}^{+}\right) \mathcal{J}_{s}^{\langle p-z\rangle} \\
& +\frac{1}{p} \sum_{z=1}^{p} \sum_{w=0}^{z} \sum_{k=1}^{p-z}(-1)^{z+k}\left(\begin{array}{c}
z \\
w
\end{array}\right)(k+1)(-Q)^{w} \mathcal{J}_{z s+w} \mathcal{J}_{s}^{\langle p-z-k\rangle} \mathcal{X}_{t}^{+((k) ; s)} .
\end{aligned}
$$


Applying the assumption of the induction again, we have

$$
\begin{aligned}
\mathcal{J}_{s}^{\langle p\rangle} \mathcal{X}_{t}^{+}= & \mathcal{X}_{t}^{+} \frac{1}{p} \sum_{z=1}^{p}(-1)^{z-1} \sum_{w=0}^{z}\left(\begin{array}{c}
z \\
w
\end{array}\right)(-Q)^{w} \mathcal{J}_{z s+w} \mathcal{J}_{s}^{\langle p-z\rangle} \\
& +2 \frac{1}{p} \sum_{z=1}^{p}(-1)^{z-1} \sum_{w=0}^{z}\left(\begin{array}{c}
z \\
w
\end{array}\right)(-Q)^{w} \mathcal{J}_{s}^{\langle p-z\rangle} \mathcal{X}_{t+z s+w}^{+} \\
& -2 \frac{1}{p} \sum_{z=1}^{p}(-1)^{z-1} \sum_{w=0}^{z}\left(\begin{array}{c}
z \\
w
\end{array}\right)(-Q)^{w} \sum_{k=1}^{p-z}(-1)^{k+1}(k+1) \mathcal{J}_{s}^{\langle p-z-k\rangle} \mathcal{X}_{t+z s+w}^{+((k) ; s)} \\
& +\frac{1}{p} \sum_{z=1}^{p} \sum_{w=0}^{z} \sum_{k=1}^{p-z}(-1)^{z+k}\left(\begin{array}{c}
z \\
w
\end{array}\right)(k+1)(-Q)^{w} \mathcal{J}_{z s+w} \mathcal{J}_{s}^{\langle p-z-k\rangle} \mathcal{X}_{t}^{+((k) ; s)} .
\end{aligned}
$$

By the definition (4.2.2), we have

$$
\mathcal{X}_{t}^{+} \frac{1}{p} \sum_{z=1}^{p}(-1)^{z-1} \sum_{w=0}^{z}\left(\begin{array}{c}
z \\
w
\end{array}\right)(-Q)^{w} \mathcal{J}_{z s+w} \mathcal{J}_{s}^{\langle p-z\rangle}=\mathcal{X}_{t}^{+} \mathcal{J}_{s}^{\langle p\rangle} .
$$

By the definition (4.2.1), we have

$$
\sum_{z=1}^{p}(-1)^{z-1} \sum_{w=0}^{z}\left(\begin{array}{c}
z \\
w
\end{array}\right)(-Q)^{w} \mathcal{J}_{s}^{\langle p-z\rangle} \mathcal{X}_{t+z s+w}^{+}=\sum_{z=1}^{p}(-1)^{z-1} \mathcal{J}_{s}^{\langle p-z\rangle} \mathcal{X}_{t}^{+((z) ; s)}
$$

Put

$$
(*)=\sum_{z=1}^{p}(-1)^{z-1} \sum_{w=0}^{z}\left(\begin{array}{c}
z \\
w
\end{array}\right)(-Q)^{w} \sum_{k=1}^{p-z}(-1)^{k+1}(k+1) \mathcal{J}_{s}^{\langle p-z-k\rangle} \mathcal{X}_{t+z s+w}^{+((k) ; s)} .
$$

By the definition (4.2.1), we also have

$$
\begin{aligned}
(*)= & \sum_{z=1}^{p}(-1)^{z-1} \sum_{w=0}^{z}\left(\begin{array}{c}
z \\
w
\end{array}\right)(-Q)^{w} \sum_{k=1}^{p-z}(-1)^{k+1}(k+1) \mathcal{J}_{s}^{\langle p-z-k\rangle} \\
& \times\left(\sum_{l=0}^{k}\left(\begin{array}{c}
k \\
l
\end{array}\right)(-Q)^{l} \mathcal{X}_{(t+z s+w)+k s+l}^{+}\right) \\
= & \sum_{z=1}^{p} \sum_{k=1}^{p-z}(-1)^{z+k}(k+1) \mathcal{J}_{s}^{\langle p-(z+k)\rangle} \sum_{w=0}^{z} \sum_{l=0}^{k}\left(\begin{array}{c}
z \\
w
\end{array}\right)\left(\begin{array}{c}
k \\
l
\end{array}\right)(-Q)^{w+l} \mathcal{X}_{t+(z+k) s+(w+l)}^{+}
\end{aligned}
$$


Put $z^{\prime}=z+k$ and $w^{\prime}=w+l$, we have

$$
(*)=\sum_{z^{\prime}=2}^{p} \sum_{k=1}^{z^{\prime}-1}(-1)^{z^{\prime}}(k+1) \mathcal{J}_{s}^{\left\langle p-z^{\prime}\right\rangle} \sum_{w^{\prime}=0}^{z^{\prime}} \sum_{l=\max \left\{0, w^{\prime}-\left(z^{\prime}-k\right)\right\}}^{\min \left\{k, w^{\prime}\right\}}\left(\begin{array}{c}
z^{\prime}-k \\
w^{\prime}-l
\end{array}\right)\left(\begin{array}{l}
k \\
l
\end{array}\right)(-Q)^{w^{\prime}} \mathcal{X}_{t+z^{\prime} s+w^{\prime}}^{+}
$$

By the induction on $k$, we can show that

$$
\sum_{l=\max \left\{0, w^{\prime}-\left(z^{\prime}-k\right)\right\}}^{\min \left\{k, w^{\prime}\right\}}\left(\begin{array}{c}
z^{\prime}-k \\
w^{\prime}-l
\end{array}\right)\left(\begin{array}{l}
k \\
l
\end{array}\right)=\left(\begin{array}{c}
z^{\prime} \\
w^{\prime}
\end{array}\right)
$$

Then, we have

$$
(*)=\sum_{z^{\prime}=2}^{p}(-1)^{z^{\prime}}\left(\sum_{k=1}^{z^{\prime}-1}(k+1)\right) \mathcal{J}_{s}^{\left\langle p-z^{\prime}\right\rangle} \sum_{w^{\prime}=0}^{z^{\prime}}\left(\begin{array}{c}
z^{\prime} \\
w^{\prime}
\end{array}\right)(-Q)^{w^{\prime}} \mathcal{X}_{t+z^{\prime} s+w^{\prime}}^{+}
$$

and by the definition of (4.2.1), we have

$$
(*)=\sum_{z^{\prime}=2}^{p}(-1) \frac{z^{\prime}}{2} \frac{\left(z^{\prime}-1\right)\left(z^{\prime}+2\right)}{2} \mathcal{J}_{s}^{\left\langle p-z^{\prime}\right\rangle} \mathcal{X}_{t}^{+\left(\left(z^{\prime}\right) ; s\right)}
$$

By the definition (4.2.2), we have

$$
\begin{aligned}
& \sum_{z=1}^{p} \sum_{w=0}^{z} \sum_{k=1}^{p-z}(-1)^{z+k}\left(\begin{array}{c}
z \\
w
\end{array}\right)(k+1)(-Q)^{w} \mathcal{J}_{z s+w} \mathcal{J}_{s}^{\langle p-z-k\rangle} \mathcal{X}_{t}^{+((k) ; s)} \\
& =\sum_{k=1}^{p-1} \sum_{z=1}^{p-k} \sum_{w=0}^{z}(-1)^{z+k}\left(\begin{array}{c}
z \\
w
\end{array}\right)(k+1)(-Q)^{w} \mathcal{J}_{z s+w} \mathcal{J}_{s}^{\langle p-z-k\rangle} \mathcal{X}_{t}^{+((k) ; s)} \\
& =\sum_{k=1}^{p-1}(-1)^{k+1}(k+1)(p-k)\left(\frac{1}{p-k} \sum_{z=1}^{p-k}(-1)^{z-1} \sum_{w=0}^{z}\left(\begin{array}{c}
z \\
w
\end{array}\right)(-Q)^{w} \mathcal{J}_{z s+w} \mathcal{J}_{s}^{\langle(p-k)-z\rangle}\right) \mathcal{X}_{t}^{+((k) ; s)} \\
& =\sum_{k=1}^{p-1}(-1)^{k+1}(k+1)(p-k) \mathcal{J}_{s}^{\langle p-k\rangle} \mathcal{X}_{t}^{+((k) ; s)} .
\end{aligned}
$$

Combining (4.3.1) with (4.3.2), (4.3.3), (4.3.5) and (4.3.6), we have

$$
\begin{aligned}
\mathcal{J}_{s}^{\langle p\rangle} \mathcal{X}_{t}^{+} & =\mathcal{X}_{t}^{+} \mathcal{J}_{s}^{\langle p\rangle}+\frac{1}{p} \sum_{z=1}^{p}(-1)^{z+1}(2+(z-1)(z+2)+(z+1)(p-z)) \mathcal{J}_{s}^{\langle p-z\rangle} \mathcal{X}_{t}^{+((z) ; s)} \\
& =\mathcal{X}_{t}^{+} \mathcal{J}_{s}^{\langle p\rangle}+\sum_{z=1}^{p}(-1)^{z+1}(z+1) \mathcal{J}_{s}^{\langle p-z\rangle} \mathcal{X}_{t}^{+((z) ; s)} .
\end{aligned}
$$


Lemma 4.4. For $s, t, h \in \mathbb{Z}_{\geq 0}$ and $p \in \mathbb{Z}_{>0}$, we have

$$
\left[\mathcal{X}_{t}^{+}, \mathcal{X}_{s}^{-((p) ; h)}\right]=\sum_{w=0}^{p}\left(\begin{array}{c}
p \\
w
\end{array}\right)(-Q)^{w} \mathcal{J}_{s+t+p h+w}^{\langle 1\rangle}
$$

Proof. By the definitions (4.2.1), (4.2.2) and the defining relation (L3), we have

$$
\begin{aligned}
\mathcal{X}_{t}^{+} \mathcal{X}_{s}^{-((p) ; h)} & =\sum_{w=0}^{p}\left(\begin{array}{c}
p \\
w
\end{array}\right)(-Q)^{w} \mathcal{X}_{t}^{+} \mathcal{X}_{s+p h+w}^{-} \\
& =\sum_{w=0}^{p}\left(\begin{array}{c}
p \\
w
\end{array}\right)(-Q)^{w}\left(\mathcal{X}_{s+p h+w}^{-} \mathcal{X}_{t}^{+}+\mathcal{J}_{s+t+p h+w}+(-Q) \mathcal{J}_{s+t+p h+w+1}\right) \\
& =\mathcal{X}_{s}^{-((p) ; h)} \mathcal{X}_{t}^{+}+\sum_{w=0}^{p}\left(\begin{array}{c}
p \\
w
\end{array}\right)(-Q)^{w} \mathcal{J}_{s+t+p h+w}^{\langle 1\rangle}
\end{aligned}
$$

Lemma 4.5. For $s, t, h \in \mathbb{Z}_{\geq 0}$ and $p \in \mathbb{Z}_{>0}$, we have the following relations.

(i) $\left[\mathcal{J}_{s}^{\langle 1\rangle}, \mathcal{X}_{t}^{+((p) ; h)}\right]=2 \mathcal{X}_{s+t-h}^{+((p+1) ; h)}$.

(ii) $\left[\mathcal{J}_{s}^{\langle 1\rangle}, \mathcal{X}_{t}^{-((p) ; h)}\right]=-2 \mathcal{X}_{s+t-h}^{-((p+1) ; h)}$.

Proof. (ii) follows from (i) by applying the algebra anti-automorphism $\dagger$ defined in (4.1.1). Then, we prove (i).

By the definition (4.2.1), we have

$$
\mathcal{J}_{s}^{\langle 1\rangle} \mathcal{X}_{t}^{+((p) ; h)}=\sum_{w=0}^{p}\left(\begin{array}{c}
p \\
w
\end{array}\right)(-Q)^{w} \mathcal{J}_{s}^{\langle 1\rangle} \mathcal{X}_{t+p h+w}^{+}
$$

Applying Lemma 4.3 (i), we have

$$
\mathcal{J}_{s}^{\langle 1\rangle} \mathcal{X}_{t}^{+((p) ; h)}=\sum_{w=0}^{p}\left(\begin{array}{c}
p \\
w
\end{array}\right)(-Q)^{w}\left(\mathcal{X}_{t+p h+w}^{+} \mathcal{J}_{s}^{\langle 1\rangle}+2 \mathcal{X}_{t+p h+w}^{+((1) ; s)}\right)
$$

Then, by the definition (4.2.1) again, we have

$$
\mathcal{J}_{s}^{\langle 1\rangle} \mathcal{X}_{t}^{+((p) ; h)}=\mathcal{X}_{t}^{+((p) ; h)} \mathcal{J}_{s}^{\langle 1\rangle}+2 \sum_{w=0}^{p}\left(\begin{array}{c}
p \\
w
\end{array}\right)(-Q)^{w}\left(\mathcal{X}_{s+t+p h+w}^{+}+(-Q) \mathcal{X}_{s+t+p h+w+1}^{+}\right)
$$

On the other hand, we have

$$
\sum_{w=0}^{p}\left(\begin{array}{c}
p \\
w
\end{array}\right)(-Q)^{w}\left(\mathcal{X}_{s+t+p h+w}^{+}+(-Q) \mathcal{X}_{s+t+p h+w+1}^{+}\right)
$$




$$
\begin{aligned}
& =\mathcal{X}_{s+t+p h}^{+}+\sum_{w=1}^{p}\left\{\left(\begin{array}{c}
p \\
w
\end{array}\right)+\left(\begin{array}{c}
p \\
w-1
\end{array}\right)\right\}(-Q)^{w} \mathcal{X}_{s+t+p h+w}^{+}+(-Q)^{p+1} \mathcal{X}_{s+t+p h+p+1}^{+} \\
& =\sum_{w=0}^{p+1}\left(\begin{array}{c}
p+1 \\
w
\end{array}\right)(-Q)^{w} \mathcal{X}_{s+t-h+(p+1) h+w}^{+} \\
& =\mathcal{X}_{s+t-h}^{+(p+1) ; h}
\end{aligned}
$$

Thus, we have (i).

Lemma 4.6. For $s, t, c \in \mathbb{Z}_{\geq 0}$, we have

$$
\left[\mathcal{X}_{t}^{+}, \mathcal{X}_{s}^{-(c)}\right]=\mathcal{X}_{s}^{-(c-1)} \mathcal{J}_{s+t}^{\langle 1\rangle}-\mathcal{X}_{s}^{-(c-2)} \mathcal{X}_{s}^{-((1) ; s+t)}
$$

Proof. We prove the lemma by the induction on $c$. If $c=0$, it is clear. If $c=1$, it is the defining relation (L3). If $c>1$, by the assumption of the induction, we have

$$
\begin{aligned}
\mathcal{X}_{t}^{+} \mathcal{X}_{s}^{-(c)} & =\frac{1}{c} \mathcal{X}_{t}^{+} \mathcal{X}_{s}^{-(c-1)} \mathcal{X}_{s}^{-} \\
& =\frac{1}{c}\left(\mathcal{X}_{s}^{-(c-1)} \mathcal{X}_{t}^{+}+\mathcal{X}_{s}^{-(c-2)} \mathcal{J}_{s+t}^{\langle 1\rangle}-\mathcal{X}_{s}^{-(c-3)} \mathcal{X}_{s}^{-((1) ; s+t)}\right) \mathcal{X}_{s}^{-}
\end{aligned}
$$

Then, by the defining relations (L3), (L4) and Lemma 4.3 (ii), we have

$$
\begin{aligned}
& \mathcal{X}_{t}^{+} \mathcal{X}_{s}^{-(c)}= \frac{1}{c}\left\{\mathcal{X}_{s}^{-(c-1)}\left(\mathcal{X}_{s}^{-} \mathcal{X}_{t}^{+}+\mathcal{J}_{s+t}^{\langle 1\rangle}\right)+\mathcal{X}_{s}^{-(c-2)}\left(\mathcal{X}_{s}^{-} \mathcal{J}_{s+t}^{\langle 1\rangle}-2 \mathcal{X}_{s}^{-((1) ; s+t)}\right)\right. \\
&\left.\quad-\mathcal{X}_{s}^{-(c-3)} \mathcal{X}_{s}^{-} \mathcal{X}_{s}^{-((1) ; s+t)}\right\} \\
&=\frac{1}{c}\left\{c \mathcal{X}_{s}^{-(c)} \mathcal{X}_{t}^{+}+\mathcal{X}_{s}^{-(c-1)} \mathcal{J}_{s+t}^{\langle 1\rangle}+(c-1) \mathcal{X}_{s}^{-(c-1)} \mathcal{J}_{s+t}^{\langle 1\rangle}\right. \\
&\left.\quad-2 \mathcal{X}_{s}^{-(c-2)} \mathcal{X}_{s}^{-((1) ; s+t)}-(c-2) \mathcal{X}_{s}^{-(c-2)} \mathcal{X}_{s}^{-((1) ; s+t)}\right\} \\
&=\mathcal{X}_{s}^{-(c)} \mathcal{X}_{t}^{+}+\mathcal{X}_{s}^{-(c-1)} \mathcal{J}_{s+t}^{\langle 1\rangle}-\mathcal{X}_{s}^{-(c-2)} \mathcal{X}_{s}^{-((1) ; s+t)}
\end{aligned}
$$

4.7. A partition $\lambda=\left(\lambda_{1}, \lambda_{2}, \ldots\right)$ is a non-increasing sequence of non-negative integers which has only finitely many non-zero terms. The size of a partition $\lambda$ is the sum of all terms of $\lambda$, and we denote it by $|\lambda|$. Namely, we have $|\lambda|=\sum_{i \geq 1} \lambda_{i}$. If $|\lambda|=n$, we say that $\lambda$ is a partition of $n$, and we denote it by $\lambda \vdash n$. The length of $\lambda$ is the maximal $i$ such that $\lambda_{i} \neq 0$, and we denote the length of $\lambda$ by $\ell(\lambda)$. For a partition $\lambda=\left(\lambda_{1}, \lambda_{2}, \ldots\right)$, let $m_{j}(\lambda)\left(j \in \mathbb{Z}_{>0}\right)$ be the multiplicity of $j$ in $\lambda$. Then, for a partition $\lambda$ and $t, h \in \mathbb{Z}_{\geq 0}$, we define an element $\mathcal{X}_{t}^{+(\lambda ; h)}$ (resp. $\mathcal{X}_{t}^{-(\lambda ; h)}$ ) of $U\left(\mathfrak{s i}_{2}^{\langle Q\rangle}[x]\right)$ by

$$
\mathcal{X}_{t}^{ \pm(\lambda ; h)}=\prod_{j \geq 1} \frac{\left(\mathcal{X}_{t}^{ \pm((j) ; h)}\right)^{m_{j}(\lambda)}}{m_{j}(\lambda) !}
$$


where we note the defining relation (L4). Clearly, we have $\dagger\left(\mathcal{X}_{t}^{+(\lambda ; h)}\right)=\mathcal{X}_{t}^{-(\lambda ; h)}$. For examples, we have

$$
\begin{aligned}
& \mathcal{X}_{t}^{ \pm((0) ; h)}=1, \quad \mathcal{X}_{t}^{ \pm((1) ; h)}=\mathcal{X}_{t}^{ \pm((1) ; h)} \\
& \mathcal{X}_{t}^{ \pm((2) ; h)}=\mathcal{X}_{t}^{ \pm((2) ; h)}, \quad \mathcal{X}_{t}^{ \pm((1,1) ; h)}=\frac{\left(\mathcal{X}_{t}^{ \pm((1) ; h)}\right)^{2}}{2 !} \\
& \mathcal{X}_{t}^{ \pm((3) ; h)}=\mathcal{X}_{t}^{ \pm((3) ; h)}, \quad \mathcal{X}_{t}^{ \pm((2,1) ; h)}=\mathcal{X}_{t}^{ \pm((2) ; h)} \mathcal{X}_{t}^{ \pm((1) ; h)}, \quad \mathcal{X}_{t}^{ \pm((1,1,1) ; h)}=\frac{\left(\mathcal{X}_{t}^{ \pm(1) ; h)}\right)^{3}}{3 !} \\
& \mathcal{X}_{t}^{ \pm((3,3,2,2,2,1,1) ; h)}=\frac{\left(\mathcal{X}_{t}^{ \pm((3) ; h)}\right)^{2}}{2 !} \frac{\left(\mathcal{X}_{t}^{ \pm((2) ; h)}\right)^{3}}{3 !} \frac{\left(\mathcal{X}_{t}^{+((1) ; h)}\right)^{2}}{2 !}
\end{aligned}
$$

For $t, h, k, b, p \in \mathbb{Z}_{\geq 0}$, we define an element $\mathcal{X}_{t}^{+(b ; p \mid k ; h)}\left(\operatorname{resp.} \quad \mathcal{X}_{t}^{-(b ; p \mid k ; h)}\right)$ of $U\left(\mathfrak{s i}_{2}^{\langle Q\rangle}[x]\right)$ by

$$
\mathcal{X}_{t}^{ \pm(b ; p \mid k ; h)}=\sum_{\lambda \vdash k} \mathcal{X}_{t}^{ \pm(\lambda ; h)} \mathcal{X}_{t}^{ \pm(b-p-\ell(\lambda))}
$$

Note the defining relation (L4), we see that $\dagger\left(\mathcal{X}_{t}^{+(b ; p \mid k ; h)}\right)=\mathcal{X}_{t}^{-(b ; p \mid k ; h)}$. For examples, we have

$$
\begin{aligned}
& \mathcal{X}_{t}^{ \pm(b ; p \mid 0 ; h)}=\mathcal{X}_{t}^{ \pm(b-p)}, \quad \mathcal{X}_{t}^{ \pm(b ; p \mid 1 ; h)}=\mathcal{X}_{t}^{ \pm((1) ; h)} \mathcal{X}_{t}^{ \pm(b-p-1)} \\
& \mathcal{X}_{t}^{ \pm(b ; p \mid 2 ; h)}=\mathcal{X}_{t}^{ \pm((2) ; h)} \mathcal{X}_{t}^{ \pm(b-p-1)}+\mathcal{X}_{t}^{ \pm((1,1) ; h)} \mathcal{X}_{t}^{ \pm(b-p-2)} \\
& \mathcal{X}_{t}^{ \pm(b ; p \mid 3 ; h)}=\mathcal{X}_{t}^{ \pm((3) ; h)} \mathcal{X}_{t}^{ \pm(b-p-1)}+\mathcal{X}_{t}^{ \pm((2,1) ; h)} \mathcal{X}_{t}^{ \pm(b-p-2)}+\mathcal{X}_{t}^{ \pm((1,1,1) ; h)} \mathcal{X}_{t}^{ \pm(b-p-3)}
\end{aligned}
$$

For the element $\mathcal{X}_{t}^{ \pm(b ; p \mid k ; h)} \in U\left(\mathfrak{s l}_{2}^{\langle Q\rangle}[x]\right)$, we prepare the following technical formulas.

Lemma 4.8. For $t, h, k, b, p \in \mathbb{Z}_{\geq 0}$, we have the following equations for the element $\mathcal{X}_{t}^{ \pm(b ; p \mid k ; h)}$ of $U\left(\mathfrak{s l}_{2}^{\langle Q\rangle}[x]\right)$.

(i) If $b-p<0$, we have $\mathcal{X}_{t}^{ \pm(b ; p \mid k ; h)}=0$.

(ii) If $k=0$, we have $\mathcal{X}_{t}^{ \pm(b ; p \mid 0 ; h)}=\mathcal{X}_{t}^{ \pm(b-p)}$.

If $k=1$, we have $\mathcal{X}_{t}^{ \pm(b ; p \mid 1 ; h)}=\mathcal{X}_{t}^{ \pm((1) ; h)} \mathcal{X}_{t}^{ \pm(b-p-1)}$.

(iii) If $p=b$, we have $\mathcal{X}_{t}^{ \pm(b ; b \mid k ; h)}= \begin{cases}1 & \text { if } k=0, \\ 0 & \text { if } k \neq 0 .\end{cases}$

(iv) If $b, p>0$, we have $\mathcal{X}_{t}^{ \pm(b ; p \mid k ; h)}=\mathcal{X}_{t}^{ \pm(b-1 ; p-1 \mid k ; h)}$.

(v) If $b, k>0$, we have

$$
\mathcal{X}_{t}^{ \pm(b ; p \mid k ; h)}=\frac{1}{k} \sum_{z=1}^{k} z \mathcal{X}_{t}^{ \pm((z) ; h)} \mathcal{X}_{t}^{ \pm(b-1 ; p \mid k-z ; h)}
$$


(vi) If $b>0$, we have

$$
(b-p+k) \mathcal{X}_{t}^{ \pm(b ; p \mid k ; h)}=\mathcal{X}_{t}^{ \pm} \mathcal{X}_{t}^{ \pm(b-1 ; p \mid k ; h)}+\sum_{z=1}^{k}(z+1) \mathcal{X}_{t}^{ \pm((z) ; h)} \mathcal{X}_{t}^{ \pm(b-1 ; p \mid k-z ; h)}
$$

Proof. (i), (ii), (iii) and (iv) are clear from definitions.

We prove $(\mathrm{v})$. Note that $\sum_{z \geq 1} z m_{z}(\lambda)=k$ for a partition $\lambda$ of $k$. Then, by the definition (4.7.2), we have

$$
\mathcal{X}_{t}^{ \pm(b ; p \mid k ; h)}=\sum_{\lambda \vdash k} \mathcal{X}_{t}^{ \pm(\lambda ; h)} \mathcal{X}_{t}^{ \pm(b-p-\ell(\lambda))}=\frac{1}{k} \sum_{\lambda \vdash k}\left(\sum_{z \geq 1} z m_{z}(\lambda)\right) \mathcal{X}_{t}^{ \pm(\lambda ; h)} \mathcal{X}_{t}^{ \pm(b-p-\ell(\lambda))}
$$

On the other hand, by the definition (4.7.1), we have

$$
\mathcal{X}_{t}^{ \pm(\lambda ; h)}=\prod_{j \geq 1} \frac{\left(\mathcal{X}_{t}^{ \pm((j) ; h)}\right)^{m_{j}(\lambda)}}{m_{j}(\lambda) !}=\frac{1}{m_{z}(\lambda)} \mathcal{X}_{t}^{ \pm((z) ; h)} \frac{\left(\mathcal{X}_{t}^{ \pm((z) ; h)}\right)^{m_{z}(\lambda)-1}}{\left(m_{z}(\lambda)-1\right) !} \prod_{\substack{j \geq 1 \\ j \neq z}} \frac{\left(\mathcal{X}_{t}^{ \pm((j) ; h)}\right)^{m_{j}(\lambda)}}{m_{j}(\lambda) !}
$$

for each $z$ such that $m_{z}(\lambda) \neq 0$. Thus, we have

$$
\begin{aligned}
\mathcal{X}_{t}^{ \pm(b ; p \mid k ; h)} & =\frac{1}{k} \sum_{\lambda \vdash k} \sum_{\substack{z \geq 1 \\
m z(\lambda) \neq 0}} z \mathcal{X}_{t}^{ \pm((z) ; h)} \frac{\left(\mathcal{X}_{t}^{ \pm((z) ; h)}\right)^{m_{z}(\lambda)-1}}{\left(m_{z}(\lambda)-1\right) !} \prod_{\substack{j \geq 1 \\
j \neq z}} \frac{\left(\mathcal{X}_{t}^{ \pm((j) ; h)}\right)^{m_{j}(\lambda)}}{m_{j}(\lambda) !} \mathcal{X}_{t}^{ \pm(b-p-\ell(\lambda))} \\
& =\frac{1}{k} \sum_{z=1}^{k} z \mathcal{X}_{t}^{ \pm((z) ; h)} \sum_{\substack{\lambda \vdash k \\
m_{z}(\lambda) \neq 0}} \frac{\left(\mathcal{X}_{t}^{ \pm((z) ; h)}\right)^{m_{z}(\lambda)-1}}{\left(m_{z}(\lambda)-1\right) !} \prod_{\substack{j \geq 1 \\
j \neq z}} \frac{\left(\mathcal{X}_{t}^{ \pm((j) ; h)}\right)^{m_{j}(\lambda)}}{m_{j}(\lambda) !} \mathcal{X}_{t}^{ \pm(b-p-\ell(\lambda))} \\
& =\frac{1}{k} \sum_{z=1}^{k} z \mathcal{X}_{t}^{ \pm((z) ; h)} \sum_{\substack{\mu \vdash k-z j \geq 1\\
}} \frac{\left(\mathcal{X}_{t}^{ \pm((j) ; h)}\right)^{m_{j}(\mu)}}{m_{j}(\mu) !} \mathcal{X}_{t}^{ \pm(b-p-(\ell(\mu)+1))} \\
& =\frac{1}{k} \sum_{z=1}^{k} z \mathcal{X}_{t}^{ \pm((z) ; h)} \sum_{\mu \vdash k-z} \mathcal{X}_{t}^{ \pm(\mu ; h)} \mathcal{X}_{t}^{ \pm((b-1)-p-\ell(\mu))} \\
& =\frac{1}{k} \sum_{z=1}^{k} z \mathcal{X}_{t}^{ \pm((z) ; h)} \mathcal{X}_{t}^{ \pm(b-1 ; p \mid k-z ; h)} .
\end{aligned}
$$

We prove (vi). By the definition (4.7.2), we have

$$
\begin{aligned}
& (b-p+k) \mathcal{X}_{t}^{ \pm(b ; p \mid k ; h)} \\
& =k \mathcal{X}_{t}^{ \pm(b ; p \mid k ; h)}+\sum_{\lambda \vdash k} \ell(\lambda) \mathcal{X}_{t}^{ \pm(\lambda ; h)} \mathcal{X}_{t}^{ \pm(b-p-\ell(\lambda))}+\sum_{\lambda \vdash k}(b-p-\ell(\lambda)) \mathcal{X}_{t}^{ \pm(\lambda ; h)} \mathcal{X}_{t}^{ \pm(b-p-\ell(\lambda))} .
\end{aligned}
$$


Note that $\ell(\lambda)=\sum_{z \geq 1} m_{z}(\lambda),(b-p-\ell(\lambda)) \mathcal{X}_{t}^{ \pm(b-p-\ell(\lambda))}=\mathcal{X}_{t}^{ \pm} \mathcal{X}_{t}^{ \pm(b-p-\ell(\lambda)-1)}$ and the defining relation (L4), we have

$$
\begin{aligned}
& (b-p+k) \mathcal{X}_{t}^{ \pm(b ; p \mid k ; h)} \\
& =k \mathcal{X}_{t}^{ \pm(b ; p \mid k ; h)}+\sum_{\lambda \vdash k}\left(\sum_{z \geq 1} m_{z}(\lambda)\right) \mathcal{X}_{t}^{ \pm(\lambda ; h)} \mathcal{X}_{t}^{ \pm(b-p-\ell(\lambda))}+\mathcal{X}_{t}^{ \pm} \sum_{\lambda \vdash k} \mathcal{X}_{t}^{ \pm(\lambda ; h)} \mathcal{X}_{t}^{ \pm(b-1-p-\ell(\lambda))}
\end{aligned}
$$

In a similar argument as in the proof of $(\mathrm{v})$, we have

$$
\begin{aligned}
& (b-p+k) \mathcal{X}_{t}^{ \pm(b ; p \mid k ; h)} \\
& =k \mathcal{X}_{t}^{ \pm(b ; p \mid k ; h)}+\sum_{z=1}^{k} \mathcal{X}_{t}^{ \pm((z) ; h)} \mathcal{X}_{t}^{ \pm(b-1 ; p \mid k-z ; h)}+\mathcal{X}_{t}^{ \pm} \sum_{\lambda \vdash k} \mathcal{X}_{t}^{ \pm(\lambda ; h)} \mathcal{X}_{t}^{ \pm((b-1)-p-\ell(\lambda))}
\end{aligned}
$$

Then, by (v) and the definition (4.7.2), we have

$$
\begin{aligned}
& (b-p+k) \mathcal{X}_{t}^{ \pm(b ; p \mid k ; h)} \\
& =\sum_{z=1}^{k} z \mathcal{X}_{t}^{ \pm((z) ; h)} \mathcal{X}_{t}^{ \pm(b-1 ; p \mid k-z ; h)}+\sum_{z=1}^{k} \mathcal{X}_{t}^{ \pm((z) ; h)} \mathcal{X}_{t}^{ \pm(b-1 ; p \mid k-z ; h)}+\mathcal{X}_{t}^{ \pm} \mathcal{X}_{t}^{ \pm(b-1 ; p \mid k ; h)} \\
& =\mathcal{X}_{t}^{ \pm} \mathcal{X}_{t}^{ \pm(b-1 ; p \mid k ; h)}+\sum_{z=1}^{k}(z+1) \mathcal{X}_{t}^{ \pm((z) ; h)} \mathcal{X}_{t}^{ \pm(b-1 ; p \mid k-z ; h)} .
\end{aligned}
$$

Lemma 4.9. For $s, t, c, p, k \in \mathbb{Z}_{\geq 0}$, we have

$$
\begin{aligned}
& {\left[\mathcal{X}_{t}^{+}, \mathcal{X}_{s}^{-(c ; p \mid k ; s+t)}\right]} \\
& =\sum_{z=0}^{k} \sum_{w=0}^{k-z}\left(\begin{array}{c}
k-z \\
w
\end{array}\right)(-Q)^{w} \mathcal{X}_{s}^{-(c ; p+1 \mid z ; s+t)} \mathcal{J}_{(k-z+1)(s+t)+w}^{\langle 1\rangle}-(k+1) \mathcal{X}_{s}^{-(c ; p+1 \mid k+1 ; s+t)} .
\end{aligned}
$$

Proof. If $c=0$, the equation (4.9.1) follows from Lemma 4.8 (i) and (iii). Then, we prove (4.9.1) by the induction on $k$ in the case where $c>0$.

If $k=0$, we see that (4.9.1) is just the formula in Lemma 4.6 by Lemma 4.8 (ii).

If $k>0$, by Lemma 4.8 (v) and the defining relation (L4), we have

$$
\mathcal{X}_{t}^{+} \mathcal{X}_{s}^{-(c ; p \mid k ; s+t)}=\frac{1}{k} \sum_{z=1}^{k} z \mathcal{X}_{t}^{+} \mathcal{X}_{s}^{-(c-1 ; p \mid k-z ; s+t)} \mathcal{X}_{s}^{-((z) ; s+t)}
$$

Applying the assumption of the induction, we have

$$
\mathcal{X}_{t}^{+} \mathcal{X}_{s}^{-(c ; p \mid k ; s+t)}
$$




$$
\begin{array}{rl}
=\frac{1}{k} \sum_{z=1}^{k} & z\left\{\mathcal{X}_{s}^{-(c-1 ; p \mid k-z ; s+t)} \mathcal{X}_{t}^{+}\right. \\
& +\sum_{y=0}^{k-z} \mathcal{X}_{s}^{-(c-1 ; p+1 \mid y ; s+t)}\left(\sum_{w=0}^{k-z-y}\left(\begin{array}{c}
k-z-y \\
w
\end{array}\right)(-Q)^{w} \mathcal{J}_{(k-z-y+1)(s+t)+w}^{\langle 1\rangle}\right) \\
& \left.-(k-z+1) \mathcal{X}_{s}^{-(c-1 ; p+1 \mid k-z+1 ; s+t)}\right\} \mathcal{X}_{s}^{-((z) ; s+t)} .
\end{array}
$$

Applying Lemma 4.4 and Lemma 4.5 (ii), we have

$$
\begin{aligned}
& \mathcal{X}_{t}^{+} \mathcal{X}_{s}^{-(c ; p \mid k ; s+t)} \\
& =\frac{1}{k} \sum_{z=1}^{k} z \mathcal{X}_{s}^{-(c-1 ; p \mid k-z ; s+t)}\left(\mathcal{X}_{s}^{-((z) ; s+t)} \mathcal{X}_{t}^{+}+\sum_{w=0}^{z}\left(\begin{array}{c}
z \\
w
\end{array}\right)(-Q)^{w} \mathcal{J}_{(z+1)(s+t)+w}^{\langle 1\rangle}\right) \\
& \quad+\frac{1}{k} \sum_{z=1}^{k} \sum_{y=0}^{k-z} z \mathcal{X}_{s}^{-(c-1 ; p+1 \mid y ; s+t)} \sum_{w=0}^{k-z-y}\left(\begin{array}{c}
k-z-y \\
w
\end{array}\right)(-Q)^{w} \\
& \quad \times\left(\mathcal{X}_{s}^{-((z) ; s+t)} \mathcal{J}_{(k-z-y+1)(s+t)+w}^{\langle 1\rangle}-2 \mathcal{X}_{s+(k-z-y)(s+t)+w}^{-((z+1) ; s+t)}\right) \\
& \quad-\frac{1}{k} \sum_{z=1}^{k} z(k-z+1) \mathcal{X}_{s}^{-(c-1 ; p+1 \mid k-z+1 ; s+t)} \mathcal{X}_{s}^{-((z) ; s+t)}
\end{aligned}
$$

Put

$$
\begin{aligned}
& (* 1)=\frac{1}{k} \sum_{z=1}^{k} z \mathcal{X}_{s}^{-(c-1 ; p \mid k-z ; s+t)} \mathcal{X}_{s}^{-((z) ; s+t)} \mathcal{X}_{t}^{+}, \\
& (* 2)=\frac{1}{k} \sum_{z=1}^{k} z \mathcal{X}_{s}^{-(c-1 ; p \mid k-z ; s+t)} \sum_{w=0}^{z}\left(\begin{array}{c}
z \\
w
\end{array}\right)(-Q)^{w} \mathcal{J}_{(z+1)(s+t)+w}^{\langle 1\rangle} \\
& (* 3)=\frac{1}{k} \sum_{z=1}^{k} \sum_{y=0}^{k-z} z \mathcal{X}_{s}^{-(c-1 ; p+1 \mid y ; s+t)} \sum_{w=0}^{k-z-y}\left(\begin{array}{c}
k-z-y \\
w
\end{array}\right)(-Q)^{w} \mathcal{X}_{s}^{-((z) ; s+t)} \mathcal{J}_{(k-z-y+1)(s+t)+w}^{\langle 1\rangle} \\
& (* 4)=\frac{1}{k} \sum_{z=1}^{k} \sum_{y=0}^{k-z} z \mathcal{X}_{s}^{-(c-1 ; p+1 \mid y ; s+t)} \sum_{w=0}^{k-z-y}\left(\begin{array}{c}
k-z-y \\
w
\end{array}\right)(-Q)^{w} \mathcal{X}_{s+(k-z-y)(s+t)+w}^{-((z+1) ; s+t)}, \\
& (* 5)=\frac{1}{k} \sum_{z=1}^{k} z(k-z+1) \mathcal{X}_{s}^{-(c-1 ; p+1 \mid k-z+1 ; s+t)} \mathcal{X}_{s}^{-((z) ; s+t)},
\end{aligned}
$$

then we have

$$
\mathcal{X}_{t}^{+} \mathcal{X}_{s}^{-(c ; p \mid k ; s+t)}=(* 1)+(* 2)+(* 3)-2(* 4)-(* 5) .
$$


By Lemma 4.8 (v) together with (L4), we have

$$
(* 1)=\mathcal{X}_{s}^{-(c ; p \mid k ; s+t)} \mathcal{X}_{t}^{+}
$$

Put $z^{\prime}=k-z$ in $(* 2)$ and apply Lemma 4.8 (iv), we have

$$
(* 2)=\frac{1}{k} \sum_{z^{\prime}=0}^{k-1}\left(k-z^{\prime}\right) \mathcal{X}_{s}^{-\left(c ; p+1 \mid z^{\prime} ; s+t\right)} \sum_{w=0}^{k-z^{\prime}}\left(\begin{array}{c}
k-z^{\prime} \\
w
\end{array}\right)(-Q)^{w} \mathcal{J}_{\left(k-z^{\prime}+1\right)(s+t)+w}^{\langle 1\rangle} .
$$

Put $h=z+y$ in $(* 3)$, we have

$$
(* 3)=\frac{1}{k} \sum_{h=1}^{k} \sum_{z=1}^{h} z \mathcal{X}_{s}^{-(c-1 ; p+1 \mid h-z ; s+t)} \mathcal{X}_{s}^{-((z) ; s+t)} \sum_{w=0}^{k-h}\left(\begin{array}{c}
k-h \\
w
\end{array}\right)(-Q)^{w} \mathcal{J}_{(k-h+1)(s+t)+w}^{\langle 1\rangle} .
$$

Applying Lemma 4.8 (v) together with (L4), we have

$$
(* 3)=\frac{1}{k} \sum_{h=1}^{k} h \mathcal{X}_{s}^{-(c ; p+1 \mid h ; s+t)} \sum_{w=0}^{k-h}\left(\begin{array}{c}
k-h \\
w
\end{array}\right)(-Q)^{w} \mathcal{J}_{(k-h+1)(s+t)+w}^{\langle 1\rangle}
$$

By (4.9.4) and (4.9.5), we have

$$
(* 2)+(* 3)=\sum_{z=0}^{k} \sum_{w=0}^{k-z}\left(\begin{array}{c}
k-z \\
w
\end{array}\right)(-Q)^{w} \mathcal{X}_{s}^{-(c ; p+1 \mid z ; s+t)} \mathcal{J}_{(k-z+1)(s+t)+w}^{\langle 1\rangle} .
$$

We also have

$$
(* 4)=\frac{1}{k} \sum_{y=0}^{k-1} \sum_{z=1}^{k-y} z \mathcal{X}_{s}^{-(c-1 ; p+1 \mid y ; s+t)} \sum_{w=0}^{k-z-y}\left(\begin{array}{c}
k-z-y \\
w
\end{array}\right)(-Q)^{w} \mathcal{X}_{s+(k-z-y)(s+t)+w}^{-((z+1) ;+t)} .
$$

Put $h=k-y+1$, we have

$$
(* 4)=\frac{1}{k} \sum_{h=2}^{k+1} \sum_{z=1}^{h-1} z \mathcal{X}_{s}^{-(c-1 ; p+1 \mid k-h+1 ; s+t)} \sum_{w=0}^{h-z-1}\left(\begin{array}{c}
h-z-1 \\
w
\end{array}\right)(-Q)^{w} \mathcal{X}_{s+(h-z-1)(s+t)+w}^{-((z+1) ; s+t)} .
$$

Put

$$
(\sharp)=\sum_{w=0}^{h-z-1}\left(\begin{array}{c}
h-z-1 \\
w
\end{array}\right)(-Q)^{w} \mathcal{X}_{s+(h-z-1)(s+t)+w}^{-((z+1) ; s+t)} .
$$


By (4.2.1), we have

$$
(\sharp)=\sum_{w=0}^{h-z-1}\left(\begin{array}{c}
h-z-1 \\
w
\end{array}\right)(-Q)^{w} \sum_{y=0}^{z+1}\left(\begin{array}{c}
z+1 \\
y
\end{array}\right)(-Q)^{y} \mathcal{X}_{s+h(s+t)+w+y}^{-} .
$$

Put $y^{\prime}=w+y$, we have

$$
(\sharp)=\sum_{y^{\prime}=0}^{h}\left(\sum_{w=\max \left\{0, y^{\prime}-(z+1)\right\}}^{\min \left\{h-(z+1), y^{\prime}\right\}}\left(\begin{array}{c}
h-(z+1) \\
w
\end{array}\right)\left(\begin{array}{c}
z+1 \\
y^{\prime}-w
\end{array}\right)\right)(-Q)^{y^{\prime}} \mathcal{X}_{s+h(s+t)+y^{\prime}}^{-}
$$

Note that $\sum_{w=\max \left\{0, y^{\prime}-(z+1)\right\}}^{\min \left\{h-(z+1), y^{\prime}\right\}}\left(\begin{array}{c}h-(z+1) \\ w\end{array}\right)\left(\begin{array}{c}z+1 \\ y^{\prime}-w\end{array}\right)=\left(\begin{array}{c}h \\ y^{\prime}\end{array}\right)$ by (4.3.4), we have

$$
(\sharp)=\sum_{y^{\prime}=0}^{h}\left(\begin{array}{l}
h \\
y^{\prime}
\end{array}\right)(-Q)^{y^{\prime}} \mathcal{X}_{s+h(s+t)+y^{\prime}}^{-}=\mathcal{X}_{s}^{-((h) ; s+t)} .
$$

(Use (4.2.1) again.) Then, we have

$$
\begin{aligned}
(* 4) & =\frac{1}{k} \sum_{h=2}^{k+1}\left(\sum_{z=1}^{h-1} z\right) \mathcal{X}_{s}^{-(c-1 ; p+1 \mid k-h+1 ; s+t)} \mathcal{X}_{s}^{-((h) ; s+t)} \\
& =\frac{1}{k} \sum_{h=2}^{k+1} \frac{h(h-1)}{2} \mathcal{X}_{s}^{-(c-1 ; p+1 \mid k-h+1 ; s+t)} \mathcal{X}_{s}^{-((h) ; s+t)}
\end{aligned}
$$

Then we have

$$
\begin{aligned}
2(* 4)+(* 5) & =\sum_{z=1}^{k+1} z \mathcal{X}_{s}^{-(c-1 ; p+1 \mid k-z+1 ; s+t)} \mathcal{X}_{s}^{-((z) ; s+t)} \\
& =(k+1) \mathcal{X}_{s}^{-(c ; p+1 \mid k+1 ; s+t)}
\end{aligned}
$$

where the last equation follows from Lemma 4.8 (v).

By (4.9.2), (4.9.3), (4.9.6) and (4.9.7), we have

$$
\begin{aligned}
\mathcal{X}_{t}^{+} & \mathcal{X}_{s}^{-(c ; p \mid k ; s+t)} \\
= & \mathcal{X}_{s}^{-(c ; p \mid k ; s+t)} \mathcal{X}_{t}^{+}+\sum_{z=0}^{k} \sum_{w=0}^{k-z}\left(\begin{array}{c}
k-z \\
w
\end{array}\right)(-Q)^{w} \mathcal{X}_{s}^{-(c ; p+1 \mid z ; s+t)} \mathcal{J}_{(k-z+1)(s+t)+w}^{\langle 1\rangle} \\
& -(k+1) \mathcal{X}_{s}^{-(c ; p+1 \mid k+1 ; s+t)} .
\end{aligned}
$$


Proposition 4.10. For $s, t, b, c \in \mathbb{Z}_{\geq 0}$, we have

$$
\left[\mathcal{X}_{t}^{+(b)}, \mathcal{X}_{s}^{-(c)}\right]=\sum_{p=1}^{\min \{b, c\}} \sum_{k=0}^{p} \sum_{l=0}^{p-k}(-1)^{k+l} \mathcal{X}_{s}^{-(c ; p \mid k ; s+t)} \mathcal{J}_{s+t}^{\langle p-(k+l)\rangle} \mathcal{X}_{t}^{+(b ; p \mid l ; s+t)}
$$

Proof. We prove (4.10.1) by the induction on $b$. If $b=1$, 4.10.1) follows from Lemma 4.6 together with Lemma 4.8 .

If $b>1$, we have

$$
\begin{aligned}
& \mathcal{X}_{t}^{+(b)} \mathcal{X}_{s}^{-(c)} \\
& =\frac{1}{b} \mathcal{X}_{t}^{+} \mathcal{X}_{t}^{(b-1)} \mathcal{X}_{s}^{-(c)} \\
& =\frac{1}{b} \mathcal{X}_{t}^{+}\left(\mathcal{X}_{s}^{-(c)} \mathcal{X}_{t}^{+(b-1)}+\sum_{p=1}^{\min \{b-1, c\}} \sum_{k=0}^{p} \sum_{l=0}^{p-k}(-1)^{k+l} \mathcal{X}_{s}^{-(c ; p \mid k ; s+t)} \mathcal{J}_{s+t}^{\langle p-(k+l)\rangle} \mathcal{X}_{t}^{+(b-1 ; p \mid l ; s+t)}\right)
\end{aligned}
$$

by the assumption of the induction. Applying Lemma 4.6 and Lemma 4.9, we have

$$
\begin{aligned}
& \mathcal{X}_{t}^{+(b)} \mathcal{X}_{s}^{-(c)} \\
& =\frac{1}{b}\left\{\left(\mathcal{X}_{s}^{-(c)} \mathcal{X}_{t}^{+}+\mathcal{X}_{s}^{-(c-1)} \mathcal{J}_{s+t}^{\langle 1\rangle}-\mathcal{X}_{s}^{-(c-2)} \mathcal{X}_{s}^{-((1) ; s+t)}\right) \mathcal{X}_{t}^{+(b-1)}\right. \\
& \quad+\sum_{p=1}^{\min \{b-1, c\}} \sum_{k=0}^{p} \sum_{l=0}^{p-k}(-1)^{k+l} \\
& \quad \times\left(\mathcal{X}_{s}^{-(c ; p \mid k ; s+t)} \mathcal{X}_{t}^{+}+\sum_{z=0}^{k} \sum_{w=0}^{k-z}\left(\begin{array}{c}
k-z \\
w
\end{array}\right)(-Q)^{w} \mathcal{X}_{s}^{-(c ; p+1 \mid z ; s+t)} \mathcal{J}_{(k-z+1)(s+t)+w}^{\langle 1\rangle}\right. \\
& \left.\left.\quad-(k+1) \mathcal{X}_{s}^{-(c ; p+1 \mid k+1 ; s+t)}\right) \mathcal{J}_{s+t}^{\langle p-(k+l)\rangle} \mathcal{X}_{t}^{+(b-1 ; p \mid l ; s+t)}\right\}
\end{aligned}
$$

On the other hand, by Lemma 4.3, we have

$$
\begin{aligned}
& \mathcal{X}_{s}^{-(c ; p \mid k ; s+t)} \mathcal{X}_{t}^{+} \mathcal{J}_{s+t}^{\langle p-(k+l)\rangle} \mathcal{X}_{t}^{+(b-1 ; p \mid l ; s+t)} \\
& =\mathcal{X}_{s}^{-(c ; p \mid k ; s+t)}\left(\mathcal{J}_{s+t}^{\langle p-(k+l)\rangle} \mathcal{X}_{t}^{+}-\sum_{z=1}^{p-(k+l)}(-1)^{z+1}(z+1) \mathcal{J}_{s+t}^{\langle p-(k+l)-z\rangle} \mathcal{X}_{t}^{+((z) ; s+t)}\right) \mathcal{X}_{t}^{+(b-1 ; p \mid l ; s+t)}
\end{aligned}
$$

Put

$$
\begin{aligned}
& (* 1)=b \mathcal{X}_{s}^{-(c)} \mathcal{X}_{t}^{+(b)}+\mathcal{X}_{s}^{-(c-1)} \mathcal{J}_{s+t}^{\langle 1\rangle} \mathcal{X}_{t}^{+(b-1)}-\mathcal{X}_{s}^{-(c-2)} \mathcal{X}_{s}^{-((1) ; s+t)} \mathcal{X}_{t}^{+(b-1)} \\
& (* 2)=\sum_{p=1}^{\min \{b, c\}} \sum_{k=0}^{p} \sum_{l=0}^{p-k}(-1)^{k+l} \mathcal{X}_{s}^{-(c ; p \mid k ; s+t)} \mathcal{J}_{s+t}^{\langle p-(k+l)\rangle} \mathcal{X}_{t}^{+} \mathcal{X}_{t}^{+(b-1 ; p \mid l ; s+t)}
\end{aligned}
$$




$$
\begin{aligned}
(* 3)= & \sum_{p=1}^{\min \{b, c\}} \sum_{k=0}^{p} \sum_{l=0}^{p-k} \sum_{z=1}^{p-(k+l)}(-1)^{k+l+z}(z+1) \\
& \times \mathcal{X}_{s}^{-(c ; p \mid k ; s+t)} \mathcal{J}_{s+t}^{\langle p-(k+l)-z\rangle} \mathcal{X}_{t}^{+((z) ; s+t)} \mathcal{X}_{t}^{+(b-1 ; p \mid l ; s+t)}, \\
(* 4)= & \sum_{p=1}^{\min \{b, c\}} \sum_{k=0}^{p} \sum_{l=0}^{p-k} \sum_{z=0}^{k} \sum_{w=0}^{k-z}(-1)^{k+l}\left(\begin{array}{c}
k-z \\
w
\end{array}\right)(-Q)^{w} \\
\times & \mathcal{X}_{s}^{-(c ; p+1 \mid z ; s+t)} \mathcal{J}_{(k-z+1)(s+t)+w}^{\langle 1\rangle} \mathcal{J}_{s+t}^{\langle p-(k+l)\rangle} \mathcal{X}_{t}^{+(b-1 ; p \mid l ; s+t)}, \\
(* 5)= & \sum_{p=1}^{\min \{b, c\}} \sum_{k=0}^{p} \sum_{l=0}^{p-k}(-1)^{k+l+1}(k+1) \mathcal{X}_{s}^{-(c ; p+1 \mid k+1 ; s+t)} \mathcal{J}_{s+t}^{\langle p-(k+l)\rangle} \mathcal{X}_{t}^{+(b-1 ; p \mid l ; s+t)},
\end{aligned}
$$

then we have

$$
\mathcal{X}_{t}^{+(b)} \mathcal{X}_{s}^{-(c)}=\frac{1}{b}\{(* 1)+(* 2)+(* 3)+(* 4)+(* 5)\}
$$

where we note that $\mathcal{X}_{t}^{+(b-1 ; p \mid l ; s+t)}=0$ if $p=b$ by Lemma 4.8 (i).

By Lemma 4.8 (ii) together with (L4), we have

$$
(* 1)=b \mathcal{X}_{s}^{-(c)} \mathcal{X}_{t}^{+(b)}+\mathcal{X}_{s}^{-(c ; 1 \mid 0 ; s+t)} \mathcal{J}_{s+t}^{\langle 1\rangle} \mathcal{X}_{t}^{+(b ; 1 \mid 0 ; s+t)}-\mathcal{X}_{s}^{-(c ; 1 \mid 1 ; s+t)} \mathcal{J}_{s+t}^{\langle 0\rangle} \mathcal{X}_{t}^{+(b ; 1 \mid 0 ; s+t)}
$$

Put $l^{\prime}=l+z$ in $(* 3)$, we have

$$
=\sum_{p=1}^{\min \{b, c\}} \sum_{k=0}^{p} \sum_{l^{\prime}=1}^{p-k} \sum_{z=1}^{l^{\prime}}(-1)^{k+l^{\prime}}(z+1) \mathcal{X}_{s}^{-(c ; p \mid k ; s+t)} \mathcal{J}_{s+t}^{\left\langle p-k-l^{\prime}\right\rangle} \mathcal{X}_{t}^{+((z) ; s+t)} \mathcal{X}_{t}^{+\left(b-1 ; p \mid l^{\prime}-z ; s+t\right)}
$$

Then we have

$$
\begin{aligned}
(* 2)+(* 3)= & \sum_{p=1}^{\min \{b, c\}} \sum_{k=0}^{p} \sum_{l=0}^{p-k}(-1)^{k+l} \mathcal{X}_{s}^{-(c ; p \mid k ; s+t)} \mathcal{J}_{s+t}^{\langle p-(k+l)\rangle} \\
& \times\left(\mathcal{X}_{t}^{+} \mathcal{X}_{t}^{+(b-1 ; p \mid l ; s+t)}+\sum_{z=1}^{l}(z+1) \mathcal{X}_{t}^{+((z) ; s+t)} \mathcal{X}_{t}^{+(b-1 ; p \mid l-z ; s+t)}\right),
\end{aligned}
$$


where we note that $\sum_{z=1}^{0} \mathcal{X}_{t}^{+((z) ; s+t)} \mathcal{X}_{t}^{+(b-1 ; p \mid l-z ; s+t)}=0$. Applying Lemma 4.8 (vi), we have

$$
\begin{aligned}
(* 2)+(* 3)= & \sum_{p=1}^{\min \{b, c\}} \sum_{k=0}^{p} \sum_{l=0}^{p-k}(-1)^{k+l}(b-p+l) \mathcal{X}_{s}^{-(c ; p \mid k ; s+t)} \mathcal{J}_{s+t}^{\langle p-(k+l)\rangle} \mathcal{X}_{t}^{+(b ; p \mid l ; s+t)} \\
= & (b-1) \mathcal{X}_{s}^{-(c ; 1 \mid 0 ; s+t)} \mathcal{J}_{s+t}^{\langle 1\rangle} \mathcal{X}_{t}^{+(b ; 1 \mid 0 ; s+t)}-b \mathcal{X}_{s}^{-(c ; 1 \mid 0 ; s+t)} \mathcal{J}_{s+t}^{\langle 0\rangle} \mathcal{X}_{t}^{+(b ; 1 \mid 1 ; s+t)} \\
& -(b-1) \mathcal{X}_{s}^{-(c ; 1 \mid 1 ; s+t)} \mathcal{J}_{s+t}^{\langle 0\rangle} \mathcal{X}_{t}^{+(b ; 1 \mid 0 ; s+t)} \\
& +\sum_{p=2}^{\min \{b, c\}} \sum_{k=0}^{p} \sum_{l=0}^{p-k}(-1)^{k+l}(b-p+l) \mathcal{X}_{s}^{-(c ; p \mid k ; s+t)} \mathcal{J}_{s+t}^{\langle p-(k+l)\rangle} \mathcal{X}_{t}^{+(b ; p \mid l ; s+t)}
\end{aligned}
$$

Put $p^{\prime}=p+1$ in $(* 4)$, we have

$$
\begin{aligned}
(* 4)=\sum_{p^{\prime}=2}^{\min \{b, c\}} & \sum_{k=0}^{p^{\prime}-1} \sum_{l=0}^{p^{\prime}-k-1} \sum_{z=0}^{k} \sum_{w=0}^{k-z}(-1)^{k+l}\left(\begin{array}{c}
k-z \\
w
\end{array}\right)(-Q)^{w} \\
& \times \mathcal{X}_{s}^{-\left(c ; p^{\prime} \mid z ; s+t\right)} \mathcal{J}_{(k-z+1)(s+t)+w}^{\langle 1\rangle} \mathcal{J}_{s+t}^{\left\langle p^{\prime}-(k+l)-1\right\rangle} \mathcal{X}_{t}^{+\left(b-1 ; p^{\prime}-1 \mid l ; s+t\right)}
\end{aligned}
$$

where we note that $\mathcal{X}_{t}^{+\left(b-1 ; p^{\prime}-1 \mid l ; s+t\right)}=0$ if $p^{\prime}=b+1$, and $\mathcal{X}_{s}^{-\left(c ; p^{\prime} \mid z ; s+t\right)}=0$ if $p^{\prime}=c+1$ by Lemma 4.8 (i). Note that

$$
\sum_{k=0}^{p-1} \sum_{l=0}^{p-k-1} \sum_{z=0}^{k}=\sum_{z=0}^{p-1} \sum_{k=z}^{p-1} \sum_{l=0}^{p-k-1}=\sum_{z=0}^{p-1} \sum_{l=0}^{p-z-1} \sum_{k=z}^{p-l-1}
$$

we have

$$
\begin{aligned}
(* 4)= & \sum_{p=2}^{\min \{b, c\}} \sum_{z=0}^{p-1} \sum_{l=0}^{p-z-1} \mathcal{X}_{s}^{-(c ; p \mid z ; s+t)} \\
& \times\left(\sum_{k=z}^{p-l-1} \sum_{w=0}^{k-z}(-1)^{k+l}\left(\begin{array}{c}
k-z \\
w
\end{array}\right)(-Q)^{w} \mathcal{J}_{(k-z+1)(s+t)+w}^{\langle 1\rangle} \mathcal{J}_{s+t}^{\langle p-(k+l)-1\rangle}\right) \mathcal{X}_{t}^{+(b-1 ; p-1 \mid l ; s+t)} .
\end{aligned}
$$

Put $k^{\prime}=k-z+1$, we have

$$
\begin{aligned}
& \sum_{k=z}^{p-l-1} \sum_{w=0}^{k-z}(-1)^{k+l}\left(\begin{array}{c}
k-z \\
w
\end{array}\right)(-Q)^{w} \mathcal{J}_{(k-z+1)(s+t)+w}^{\langle 1\rangle} \mathcal{J}_{s+t}^{\langle p-(k+l)-1\rangle} \\
& =\sum_{k^{\prime}=1}^{p-l-z} \sum_{w=0}^{k^{\prime}-1}(-1)^{k^{\prime}+z+l-1}\left(\begin{array}{c}
k^{\prime}-1 \\
w
\end{array}\right)(-Q)^{w} \mathcal{J}_{k^{\prime}(s+t)+w}^{\langle 1\rangle} \mathcal{J}_{s+t}^{\left\langle p-k^{\prime}-z-l\right\rangle} .
\end{aligned}
$$


Since $\mathcal{J}_{k^{\prime}(s+t)+w}^{\langle 1\rangle}=\mathcal{J}_{k^{\prime}(s+t)+w}+(-Q) \mathcal{J}_{k^{\prime}(s+t)+w+1}$, we see that

$$
\sum_{w=0}^{k^{\prime}-1}\left(\begin{array}{c}
k^{\prime}-1 \\
w
\end{array}\right)(-Q)^{w} \mathcal{J}_{k^{\prime}(s+t)+w}^{\langle 1\rangle}=\sum_{w=0}^{k^{\prime}}\left(\begin{array}{c}
k^{\prime} \\
w
\end{array}\right)(-Q)^{w} \mathcal{J}_{k^{\prime}(s+t)+w}
$$

Thus we have

$$
\begin{aligned}
& \sum_{k=z}^{p-l-1} \sum_{w=0}^{k-z}(-1)^{k+l}\left(\begin{array}{c}
k-z \\
w
\end{array}\right)(-Q)^{w} \mathcal{J}_{(k-z+1)(s+t)+w}^{\langle 1\rangle} \mathcal{J}_{s+t}^{\langle p-(k+l)-1\rangle} \\
& =(-1)^{z+l} \sum_{k^{\prime}=1}^{p-l-z}(-1)^{k^{\prime}-1} \sum_{w=0}^{k^{\prime}}\left(\begin{array}{l}
k^{\prime} \\
w
\end{array}\right)(-Q)^{w} \mathcal{J}_{k^{\prime}(s+t)+w} \mathcal{J}_{s+t}^{\left\langle p-k^{\prime}-z-l\right\rangle} \\
& =(-1)^{z+l}(p-l-z) \mathcal{J}_{s+t}^{\langle p-l-z\rangle},
\end{aligned}
$$

where the last equation follows from (4.2.2). Then we have

$$
\begin{aligned}
(* 4) & =\sum_{p=2}^{\min \{b, c\}} \sum_{z=0}^{p-1} \sum_{l=0}^{p-z-1}(p-l-z)(-1)^{z+l} \mathcal{X}_{s}^{-(c ; p \mid z ; s+t)} \mathcal{J}_{s+t}^{\langle p-l-z\rangle} \mathcal{X}_{t}^{+(b-1 ; p-1 \mid l ; s+t)} \\
& =\sum_{p=2}^{\min \{b, c\}} \sum_{z=0}^{p} \sum_{l=0}^{p-z}(p-l-z)(-1)^{z+l} \mathcal{X}_{s}^{-(c ; p \mid z ; s+t)} \mathcal{J}_{s+t}^{\langle p-l-z\rangle} \mathcal{X}_{t}^{+(b-1 ; p-1 \mid l ; s+t)}
\end{aligned}
$$

Applying Lemma 4.8 (iv), we have

$$
(* 4)=\sum_{p=2}^{\min \{b, c\}} \sum_{z=0}^{p} \sum_{l=0}^{p-z}(p-l-z)(-1)^{z+l} \mathcal{X}_{s}^{-(c ; p \mid z ; s+t)} \mathcal{J}_{s+t}^{\langle p-l-z\rangle} \mathcal{X}_{t}^{+(b ; p \mid l ; s+t)} .
$$

Put $p^{\prime}=p+1$ in $(* 5)$, we have

$$
(* 5)=\sum_{p^{\prime}=2}^{\min \{b, c\}} \sum_{k=0}^{p^{\prime}-1} \sum_{l=0}^{p^{\prime}-k-1}(-1)^{k+l+1}(k+1) \mathcal{X}_{s}^{-\left(c ; p^{\prime} \mid k+1 ; s+t\right)} \mathcal{J}_{s+t}^{\left\langle p^{\prime}-k-l-1\right\rangle} \mathcal{X}_{t}^{+\left(b-1 ; p^{\prime}-1 \mid l ; s+t\right)},
$$

where we note that $\mathcal{X}_{t}^{+\left(b-1 ; p^{\prime}-1 \mid l ; s+t\right)}=0$ if $p^{\prime}=b+1$, and $\mathcal{X}_{s}^{-\left(c ; p^{\prime} \mid k+1 ; s+t\right)}=0$ if $p^{\prime}=c+1$ by Lemma 4.8 (i). Put $k^{\prime}=k+1$, we have

$$
\begin{aligned}
(* 5) & =\sum_{p^{\prime}=2}^{\min \{b, c\}} \sum_{k^{\prime}=1}^{p^{\prime}} \sum_{l=0}^{p^{\prime}-k^{\prime}}(-1)^{k^{\prime}+l} k^{\prime} \mathcal{X}_{s}^{-\left(c ; p^{\prime} \mid k^{\prime} ; s+t\right)} \mathcal{J}_{s+t}^{\left\langle p^{\prime}-k^{\prime}-l\right\rangle} \mathcal{X}_{t}^{+\left(b-1 ; p^{\prime}-1 \mid l ; s+t\right)} \\
& =\sum_{p=2}^{\min \{b, c\}} \sum_{k=0}^{p} \sum_{l=0}^{p-k} k(-1)^{k+l} \mathcal{X}_{s}^{-(c ; p \mid k ; s+t)} \mathcal{J}_{s+t}^{\langle p-k-l\rangle} \mathcal{X}_{t}^{+(b-1 ; p-1 \mid l ; s+t)}
\end{aligned}
$$


Applying Lemma 4.8 (iv), we have

$$
(* 5)=\sum_{p=2}^{\min \{b, c\}} \sum_{k=0}^{p} \sum_{l=0}^{p-k} k(-1)^{k+l} \mathcal{X}_{s}^{-(c ; p \mid k ; s+t)} \mathcal{J}_{s+t}^{\langle p-k-l\rangle} \mathcal{X}_{t}^{+(b ; p \mid l ; s+t)} .
$$

By (4.10.2), (4.10.3), (4.10.4), (4.10.5) and (4.10.6), we have

$$
\mathcal{X}_{t}^{+(b)} \mathcal{X}_{s}^{-(c)}=\mathcal{X}_{s}^{-(c)} \mathcal{X}_{t}^{+(b)}+\sum_{p=1}^{\min \{b, c\}} \sum_{k=0}^{p} \sum_{l=0}^{p-k}(-1)^{k+l} \mathcal{X}_{s}^{-(c ; p \mid k ; s+t)} \mathcal{J}_{s+t}^{\langle p-(k+l)\rangle} \mathcal{X}_{t}^{+(b ; p \mid l ; s+t)}
$$

$\S$ 5. RANK 1 CASE ; Finite Dimensional Simple MOdUles of $U\left(\mathfrak{s}_{2}^{\langle Q\rangle}[x]\right)$

In this section, we classify the finite dimensional simple $U\left(\mathfrak{s l}_{2}^{\langle Q\rangle}[x]\right)$-modules.

5.1. 1-dimensional representations. First, we consider 1-dimensional representations of $\mathfrak{s l}_{2}^{\langle Q\rangle}[x]$. Let $L=\mathbb{C} v$ be a 1-dimensional $U\left(\mathfrak{s l}_{2}^{\langle Q\rangle}[x]\right)$-module with a basis $\{v\}$, then $\mathcal{J}_{t}(t \geq 0)$ acts on $v$ as a scalar multiplication. If $\mathcal{X}_{t}^{+} \cdot v \neq 0$ (resp. $\left.\mathcal{X}_{t}^{-} \cdot v \neq 0\right)$, then $\mathcal{X}_{t}^{+} \cdot v\left(\right.$ resp. $\left.\mathcal{X}_{t}^{-} \cdot v\right)$ is an eigenvector for the action of $\mathcal{J}_{0}$ whose eigenvalue is different from one of $v$ by the defining relation (L2). This is a contradiction since $L$ is 1 -dimensional. Thus, we have $\mathcal{X}_{t}^{ \pm} \cdot v=0$ for $t \geq 0$. Moreover, by the defining relation (L3), we have $\left(\mathcal{J}_{t}-Q \mathcal{J}_{t+1}\right) \cdot v=\left(\mathcal{X}_{t}^{+} \mathcal{X}_{0}^{-}-\mathcal{X}_{0}^{-} \mathcal{X}_{t}^{+}\right) \cdot v=0$. This implies that $\mathcal{J}_{t} \cdot v=0$ for $t \geq 0$ if $Q=0$, and that $\mathcal{J}_{t} \cdot v=Q^{-t} \mathcal{J}_{0} \cdot v$ for $t>0$ if $Q \neq 0$.

We define the set $\mathbb{B}^{\langle Q\rangle}$ by

$$
\mathbb{B}^{\langle Q\rangle}= \begin{cases}\{0\} & \text { if } Q=0 \\ \mathbb{C} & \text { if } Q \neq 0\end{cases}
$$

For each $\beta \in \mathbb{B}^{\langle Q\rangle}$, we can define the 1-dimensional $U\left(\mathfrak{s l}_{2}^{\langle Q\rangle}[x]\right)$-module $\mathcal{L}^{\beta}=\mathbb{C} v_{0}$ such that

$$
\mathcal{X}_{t}^{ \pm} \cdot v_{0}=0, \quad \mathcal{J}_{t} \cdot v_{0}=\left\{\begin{array}{ll}
0 & \text { if } Q=0, \\
Q^{-t} \beta v_{0} & \text { if } Q \neq 0
\end{array} \quad\left(t \in \mathbb{Z}_{\geq 0}\right)\right.
$$

by checking the defining relations of $\mathfrak{s l}_{2}^{\langle Q\rangle}[x]$. Note that $\mathcal{L}^{0}$ is the trivial representation. Now we obtain the following lemma.

Lemma 5.2. Any 1-dimensional $U\left(\mathfrak{s l}_{2}^{\langle Q\rangle}[x]\right)$-module is isomorphic to $\mathcal{L}^{\beta}$ for some $\beta \in \mathbb{B}^{\langle Q\rangle}$.

5.3. Recall from $₫ 2$, a finite dimensional simple $U\left(\mathfrak{s t}_{2}^{\langle Q\rangle}[x]\right)$-module is isomorphic to a simple highest weight module $\mathcal{L}(\mathbf{u})$ for some highest weight $\mathbf{u}=\left(u_{t}\right) \in \prod_{t \geq 0} \mathbb{C}$ (Proposition 2.6), where we omit the first index for the highest weight. Then, in 
order to classify the finite dimensional simple $U\left(\mathfrak{s l}_{2}^{\langle Q\rangle}[x]\right)$-module, it is enough to classify the highest weight $\mathbf{u}$ such that $\mathcal{L}(\mathbf{u})$ is finite dimensional.

In order to obtain a necessary condition for $\mathbf{u}$ such that $\mathcal{L}(\mathbf{u})$ is finite dimensional, we prepare the following lemma.

Lemma 5.4. Let $M$ be a finite dimensional $U\left(\mathfrak{s l}_{2}^{\langle Q\rangle}[x]\right)$-module. Take an element $v \in M$ satisfying

$$
\mathcal{X}_{t}^{+} \cdot v=0, \quad \mathcal{J}_{t} \cdot v=u_{t} v \quad\left(t \in \mathbb{Z}_{\geq 0}\right), \quad \mathcal{X}_{0}^{-(n)} \cdot v \neq 0 \text { and } \mathcal{X}_{0}^{-(n+1)} \cdot v=0
$$

for some $u_{t} \in \mathbb{C}\left(t \in \mathbb{Z}_{\geq 0}\right)$ and $n \in \mathbb{Z}_{\geq 0}$. (In fact, a such element exists by Lemma 2.3.) Then, for $s, t \in \mathbb{Z}_{\geq 0}$, we have

$$
\sum_{w=0}^{n}\left(\begin{array}{l}
n \\
w
\end{array}\right)(-Q)^{w} \mathcal{J}_{t+n s+w}^{\langle 1\rangle} \cdot v=\sum_{k=0}^{n-1}(-1)^{n-k+1}\left(\sum_{w=0}^{k}\left(\begin{array}{c}
k \\
w
\end{array}\right)(-Q)^{w} \mathcal{J}_{t+k s+w}^{\langle 1\rangle}\right) \mathcal{J}_{s}^{\langle n-k\rangle} \cdot v
$$

Proof. By the assumption $\mathcal{X}_{0}^{-(n+1)} \cdot v=0$ and Proposition 4.10, we have

$$
\begin{aligned}
0 & =\mathcal{X}_{s}^{+(n)} \mathcal{X}_{0}^{-(n+1)} \cdot v \\
& =\left(\mathcal{X}_{0}^{-(n+1)} \mathcal{X}_{s}^{+(n)}+\sum_{p=1}^{n} \sum_{k=0}^{p} \sum_{l=0}^{p-k}(-1)^{k+l} \mathcal{X}_{0}^{-(n+1 ; p \mid k ; s)} \mathcal{J}_{s}^{\langle p-(k+l)\rangle} \mathcal{X}_{s}^{+(n ; p \mid l ; s)}\right) \cdot v
\end{aligned}
$$

By the definition, we have $\mathcal{X}_{s}^{+(n ; p \mid l ; s)}=\sum_{\lambda \vdash l} \mathcal{X}_{s}^{+(\lambda ; s)} \mathcal{X}_{s}^{+(n-p-\ell(\lambda))}$. Thus, by the definition of $\mathcal{X}_{s}^{+(\lambda ; s)}$ and the assumption $\mathcal{X}_{t}^{+} \cdot v=0(t \geq 0)$, we have

$$
\mathcal{X}_{s}^{+(n ; p \mid l ; s)} \cdot v= \begin{cases}v & \text { if } l=0 \text { and } p=n \\ 0 & \text { otherwise }\end{cases}
$$

Then (5.4.1) implies that

$$
0=\sum_{k=0}^{n}(-1)^{k} \mathcal{X}_{0}^{-(n+1 ; n \mid k ; s)} \mathcal{J}_{s}^{\langle n-k\rangle} \cdot v
$$

By the definition, we have

$$
\mathcal{X}_{0}^{-(n+1 ; n \mid k ; s)}=\sum_{\lambda \vdash k} \mathcal{X}_{0}^{-(\lambda ; s)} \mathcal{X}_{0}^{-(1-\ell(\lambda))}= \begin{cases}\mathcal{X}_{0}^{-} & \text {if } k=0 \\ \mathcal{X}_{0}^{-((k) ; s)} & \text { if } k \neq 0\end{cases}
$$


Thus, we have

$$
0=\mathcal{X}_{0}^{-} \mathcal{J}_{s}^{\langle n\rangle} \cdot v+\sum_{k=1}^{n}(-1)^{k} \mathcal{X}_{0}^{-((k) ; s)} \mathcal{J}_{s}^{\langle n-k\rangle} \cdot v
$$

By multiplying $\mathcal{X}_{t}^{+}$from left to this equation, we have

$$
\begin{aligned}
0 & =\mathcal{X}_{t}^{+} \mathcal{X}_{0}^{-} \mathcal{J}_{s}^{\langle n\rangle} \cdot v+\sum_{k=1}^{n}(-1)^{k} \mathcal{X}_{t}^{+} \mathcal{X}_{0}^{-((k) ; s)} \mathcal{J}_{s}^{\langle n-k\rangle} \cdot v \\
& =\sum_{k=0}^{n}(-1)^{k}\left(\sum_{w=0}^{k}\left(\begin{array}{c}
k \\
w
\end{array}\right)(-Q)^{w} \mathcal{J}_{t+k s+w}^{\langle 1\rangle}\right) \mathcal{J}_{s}^{\langle n-k\rangle} \cdot v,
\end{aligned}
$$

where we use Lemma 4.4 and the fact $\mathcal{X}_{t}^{+} \mathcal{J}_{s}^{\langle n-k\rangle} \cdot v=0$. This implies the Lemma.

This Lemma implies the following proposition which gives a necessary condition for $\mathbf{u}$ such that $\mathcal{L}(\mathbf{u})$ is finite dimensional.

Proposition 5.5. Let $M$ be a finite dimensional $U\left(\mathfrak{s}_{2}^{\langle Q\rangle}[x]\right)$-module. Take an element $v \in M$ satisfying

$$
\mathcal{X}_{t}^{+} \cdot v=0, \quad \mathcal{J}_{t} \cdot v=u_{t} v \quad\left(t \in \mathbb{Z}_{\geq 0}\right), \quad \mathcal{X}_{0}^{-(n)} \cdot v \neq 0 \text { and } \mathcal{X}_{0}^{-(n+1)} \cdot v=0
$$

for some $u_{t} \in \mathbb{C}\left(t \in \mathbb{Z}_{\geq 0}\right)$ and $n \in \mathbb{Z}_{\geq 0}$.

(i) If $Q=0$, we have $u_{0}=n$, and there exist $\gamma_{1}, \gamma_{2}, \ldots, \gamma_{n} \in \mathbb{C}$ such that

$$
u_{t}=p_{t}\left(\gamma_{1}, \gamma_{2}, \ldots, \gamma_{n}\right) \quad(t>0)
$$

where $p_{t}\left(\gamma_{1}, \ldots, \gamma_{n}\right)=\gamma_{1}^{t}+\gamma_{2}^{t}+\cdots+\gamma_{n}^{t}$.

(ii) If $Q \neq 0$, there exist $\beta, \gamma_{1}, \gamma_{2}, \ldots, \gamma_{n} \in \mathbb{C}$ such that

$$
u_{0}=n+\beta \text { and } u_{t}=p_{t}\left(\gamma_{1}, \gamma_{2}, \ldots, \gamma_{n}\right)+Q^{-t} \beta \quad(t>0),
$$

where $p_{t}\left(\gamma_{1}, \ldots, \gamma_{n}\right)=\gamma_{1}^{t}+\gamma_{2}^{t}+\cdots+\gamma_{n}^{t}$.

Proof. (i). Assume that $Q=0$. Then, $\mathfrak{s}_{2}^{\langle 0\rangle}[x]$ coincides with the current Lie algebra $\mathfrak{s l}_{2}[x]$ of $\mathfrak{s l}_{2}$. Moreover, the Lie subalgebra of $\mathfrak{s l}_{2}[x]$ generated by $\mathcal{X}_{0}^{ \pm}$and $\mathcal{J}_{0}$ is isomorphic to $\mathfrak{s l}_{2}$. Thus, by the representation theory of $\mathfrak{s l}_{2}$, we have $u_{0}=n$.

For $u_{1}, \ldots, u_{n}$, there exist $\gamma_{1}, \ldots, \gamma_{n} \in \mathbb{C}$ such that

$$
u_{k}=p_{k}\left(\gamma_{1}, \ldots, \gamma_{n}\right) \text { for } k=1, \ldots, n
$$

by Lemma A.2. 
By the definition, we have

$$
\mathcal{J}_{1}^{\langle k\rangle}=\frac{1}{k} \sum_{z=1}^{k}(-1)^{z-1} \mathcal{J}_{z} \mathcal{J}_{1}^{\langle k-z\rangle}
$$

since we assume $Q=0$. By the induction on $k$ together with (5.5.1), (5.5.2) and (A.1.1), we can show that

$$
\mathcal{J}_{1}^{\langle k\rangle} \cdot v=e_{k}\left(\gamma_{1}, \ldots, \gamma_{n}\right) v \text { for } k=1, \ldots, n,
$$

where $e_{k}\left(\gamma_{1}, \ldots, \gamma_{n}\right)=\sum_{1 \leq i_{1}<i_{2}<\cdots<i_{k} \leq n} \gamma_{i_{1}} \gamma_{i_{2}} \ldots \gamma_{i_{k}}$.

By the induction on $t$, we prove that

$$
u_{t}=p_{t}\left(\gamma_{1}, \ldots, \gamma_{n}\right) \quad(t>0) \text {. }
$$

If $t \leq n$, (5.5.4) follows from (5.5.1). If $t>n$, by Lemma 5.4 in the case where $s=1$, we have

$$
u_{t} v=\mathcal{J}_{(t-n)+n} \cdot v=\sum_{k=0}^{n-1}(-1)^{n-k+1} \mathcal{J}_{(t-n)+k} \mathcal{J}_{1}^{\langle n-k\rangle} \cdot v
$$

By the assumption of the induction together with (5.5.3) and (A.1.2), we have

$$
u_{t} v=\sum_{k=0}^{n-1}(-1)^{n-k+1} p_{t-n+k}\left(\gamma_{1}, \ldots, \gamma_{n}\right) e_{n-k}\left(\gamma_{1}, \ldots, \gamma_{n}\right) v=p_{t}\left(\gamma_{1}, \ldots, \gamma_{n}\right) v
$$
that

(ii). Assume that $Q \neq 0$. For $u_{0}, u_{1}, \ldots, u_{n}$, there exist $\beta, \gamma_{1}, \ldots, \gamma_{n} \in \mathbb{C}$ such

$$
u_{0}=n+\beta \text { and } u_{k}=p_{k}\left(\gamma_{1}, \ldots, \gamma_{n}\right)+Q^{-k} \beta \text { for } k=1, \ldots, n
$$

by Lemma A.2.

By the induction on $k$, we prove that

$$
\mathcal{J}_{0}^{\langle k\rangle} \cdot v=e_{k}\left(\theta_{1}, \theta_{2}, \ldots, \theta_{n}\right) v \text { for } k=1, \ldots, n
$$

where $\theta_{i}=1-Q \gamma_{i}(1 \leq i \leq n)$ and $e_{k}\left(\theta_{1}, \ldots, \theta_{n}\right)=\sum_{1 \leq i_{1}<i_{2}<\cdots<i_{k} \leq n} \theta_{i_{1}} \theta_{i_{2}} \ldots \theta_{i_{k}}$.

In the case where $k=1$, we have $\mathcal{J}_{0}^{\langle 1\rangle} \cdot v=\left(\mathcal{J}_{0}+(-Q) \mathcal{J}_{1}\right) \cdot v$. Then we have $\mathcal{J}_{0}^{\langle 1\rangle} \cdot v=e_{1}\left(\theta_{1}, \ldots, \theta_{n}\right) v$ by (5.5.5).

In the case where $1<k \leq n$, by the definition, we have

$$
\mathcal{J}_{0}^{\langle k\rangle} \cdot v=\frac{1}{k} \sum_{z=1}^{k}(-1)^{z-1}\left(\sum_{w=0}^{z}\left(\begin{array}{c}
z \\
w
\end{array}\right)(-Q)^{w} \mathcal{J}_{w}\right) \mathcal{J}_{0}^{\langle k-z\rangle} \cdot v
$$


Applying the assumption of the induction to the right-hand side, we have

$$
\mathcal{J}_{0}^{\langle k\rangle} \cdot v=\frac{1}{k} \sum_{z=1}^{k}(-1)^{z-1} e_{k-z}\left(\theta_{1}, \ldots, \theta_{n}\right)\left(\sum_{w=0}^{z}\left(\begin{array}{c}
z \\
w
\end{array}\right)(-Q)^{w} \mathcal{J}_{w}\right) \cdot v
$$

where we note that $\mathcal{J}_{0}^{\langle 0\rangle}=e_{0}\left(\theta_{1}, \ldots, \theta_{n}\right)=1$. On the other hand, by (5.5.5), we have

$$
\sum_{w=0}^{z}\left(\begin{array}{c}
z \\
w
\end{array}\right)(-Q)^{w} \mathcal{J}_{w} \cdot v=p_{z}\left(\theta_{1}, \ldots, \theta_{n}\right) v \quad(1 \leq z \leq k \leq n)
$$

where we note that $\sum_{w=0}^{z}\left(\begin{array}{c}z \\ w\end{array}\right)(-1)^{w}=0$. By (5.5.7) and (5.5.8) together with (A.1.1), we have (5.5.6).

By the induction on $t$, we prove that

$$
u_{t}=p_{t}\left(\gamma_{1}, \ldots, \gamma_{n}\right)+Q^{-t} \beta \quad(t>0) .
$$

If $t \leq n$, (5.5.9) follows from (5.5.5). If $t>n$, by Lemma 5.4 in the case where $s=0$, we have

$$
\sum_{w=0}^{n}\left(\begin{array}{l}
n \\
w
\end{array}\right)(-Q)^{w} \mathcal{J}_{(t-n-1)+w}^{\langle\langle\rangle} \cdot v=\sum_{k=0}^{n-1}(-1)^{n-k+1}\left(\sum_{w=0}^{k}\left(\begin{array}{c}
k \\
w
\end{array}\right)(-Q)^{w} \mathcal{J}_{(t-n-1)+w}^{\langle\langle\rangle}\right) \mathcal{J}_{0}^{\langle n-k\rangle} \cdot v
$$

By (5.5.6), we have

$$
\begin{aligned}
& \sum_{w=0}^{n}\left(\begin{array}{c}
n \\
w
\end{array}\right)(-Q)^{w} \mathcal{J}_{(t-n-1)+w}^{\langle 1\rangle} \cdot v \\
& =\sum_{k=0}^{n-1}(-1)^{n-k+1} e_{n-k}\left(\theta_{1}, \ldots, \theta_{n}\right)\left(\sum_{w=0}^{k}\left(\begin{array}{c}
k \\
w
\end{array}\right)(-Q)^{w} \mathcal{J}_{(t-n-1)+w}^{\langle 1\rangle}\right) \cdot v .
\end{aligned}
$$

On the other hand, for $k \geq 0$, we have

$$
\sum_{w=0}^{k}\left(\begin{array}{c}
k \\
w
\end{array}\right)(-Q)^{w} \mathcal{J}_{(t-n-1)+w}^{\langle 1\rangle}=\sum_{w=0}^{k+1}\left(\begin{array}{c}
k+1 \\
w
\end{array}\right)(-Q)^{w} \mathcal{J}_{(t-n-1)+w}
$$


since $\mathcal{J}_{(t-n-1)+w}^{\langle 1\rangle}=\mathcal{J}_{(t-n-1)+w}+(-Q) \mathcal{J}_{(t-n-1)+w+1}$. Then, by (5.5.11) and the assumption of the induction, we have

$$
\begin{aligned}
& \sum_{w=0}^{n}\left(\begin{array}{c}
n \\
w
\end{array}\right)(-Q)^{w} \mathcal{J}_{(t-n-1)+w}^{\langle 1\rangle} \cdot v \\
& =(-Q)^{n+1} \mathcal{J}_{t} \cdot v+\sum_{w=0}^{n}\left(\begin{array}{c}
n+1 \\
w
\end{array}\right)(-Q)^{w}\left(p_{(t-n-1)+w}\left(\gamma_{1}, \ldots, \gamma_{n}\right)+Q^{-((t-n-1)+w)} \beta\right) v
\end{aligned}
$$

and

$$
\begin{aligned}
& \sum_{w=0}^{k}\left(\begin{array}{c}
k \\
w
\end{array}\right)(-Q)^{w} \mathcal{J}_{(t-n-1)+w}^{\langle 1\rangle} \cdot v \\
& =\sum_{w=0}^{k+1}\left(\begin{array}{c}
k+1 \\
w
\end{array}\right)(-Q)^{w}\left(p_{(t-n-1)+w}\left(\gamma_{1}, \ldots, \gamma_{n}\right)+Q^{-((t-n-1)+w)} \beta\right) v
\end{aligned}
$$

for $k=0,1, \ldots, n-1$. Moreover, by the direct calculations, we have

$$
\sum_{w=0}^{k+1}\left(\begin{array}{c}
k+1 \\
w
\end{array}\right)(-Q)^{w}\left(p_{(t-n-1)+w}\left(\gamma_{1}, \ldots, \gamma_{n}\right)+Q^{-((t-n-1)+w)} \beta\right)=p_{k+1}^{(\gamma)}\left(\theta_{1}, \ldots, \theta_{n}\right)
$$

for $k \geq 0$, where $p_{k+1}^{(\gamma)}\left(\theta_{1}, \ldots, \theta_{n}\right)=\gamma_{1}^{t-n-1} \theta_{1}^{k+1}+\gamma_{2}^{t-n-1} \theta_{2}^{k+1}+\cdots+\gamma_{n}^{t-n-1} \theta_{n}^{k+1}$. Then, by (5.5.10), (5.5.12), (5.5.13) and (5.5.14), we have

$$
\begin{aligned}
& (-Q)^{n+1} \mathcal{J}_{t} \cdot v-(-Q)^{n+1}\left(p_{t}\left(\gamma_{1}, \ldots, \gamma_{n}\right)+Q^{-t} \beta\right) v+p_{n+1}^{(\gamma)}\left(\theta_{1}, \ldots, \theta_{n}\right) \\
& =\sum_{k=0}^{n-1}(-1)^{n-k+1} e_{n-k}\left(\theta_{1}, \ldots, \theta_{n}\right) p_{k+1}^{(\gamma)}\left(\theta_{1}, \ldots, \theta_{n}\right) .
\end{aligned}
$$

Applying (A.3.2) to the right-hand side, we have

$$
\mathcal{J}_{t} \cdot v=\left(p_{t}\left(\gamma_{1}, \ldots, \gamma_{n}\right)+Q^{-t} \beta\right) v
$$

5.6. By Lemma 5.2 and Proposition 5.5, we see that the highest weight $\mathbf{u}=\left(u_{t}\right)_{t \geq 0}$ of a simple highest weight $U\left(\mathfrak{s l}_{2}^{\langle Q\rangle}[x]\right)$-module $\mathcal{L}(\mathbf{u})$ has the form

$$
u_{0}=\left\{\begin{array}{ll}
n & \text { if } Q=0 \\
n+\beta & \text { if } Q \neq 0,
\end{array} \quad u_{t}= \begin{cases}p_{t}\left(\gamma_{1}, \gamma_{2}, \ldots, \gamma_{n}\right) & \text { if } Q=0 \\
p_{t}\left(\gamma_{1}, \gamma_{2}, \ldots, \gamma_{n}\right)+Q^{-t} \beta & \text { if } Q \neq 0\end{cases}\right.
$$


for some $n \in \mathbb{Z}_{\geq 0}$ and $\beta, \gamma_{1}, \gamma_{2}, \ldots, \gamma_{n} \in \mathbb{C}$ if $\mathcal{L}(\mathbf{u})$ is finite dimensional.

Let $\mathbb{C}[x]$ be the polynomial ring over $\mathbb{C}$ with the indeterminate variable $x$, and let $\mathbb{C}[x]_{\text {monic }}$ be the subset of $\mathbb{C}[x]$ consisting of monic polynomials. We define the set $\mathbb{C}[x]_{\text {monic }}^{\langle Q\rangle}$ by

$$
\mathbb{C}[x]_{\text {monic }}^{\langle Q\rangle}= \begin{cases}\mathbb{C}[x]_{\text {monic }} & \text { if } Q=0 \\ \left\{\varphi \in \mathbb{C}[x]_{\text {monic }} \mid Q^{-1} \text { is not a root of } \varphi\right\} & \text { if } Q \neq 0\end{cases}
$$

Recall that

$$
\mathbb{B}^{\langle Q\rangle}= \begin{cases}\{0\} & \text { if } Q=0 \\ \mathbb{C} & \text { if } Q \neq 0\end{cases}
$$

We define the map

$$
\mathbb{C}[x]_{\text {monic }}^{\langle Q\rangle} \times \mathbb{B}^{\langle Q\rangle} \rightarrow \prod_{t \geq 0} \mathbb{C}, \quad(\varphi, \beta) \mapsto \mathbf{u}^{\langle Q\rangle}(\varphi, \beta)=\left(\mathbf{u}^{\langle Q\rangle}(\varphi, \beta)_{t}\right)_{t \geq 0}
$$

by

$$
\mathbf{u}^{\langle Q\rangle}(\varphi, \beta)_{t}= \begin{cases}\operatorname{deg} \varphi+\beta & \text { if } t=0 \\ p_{t}\left(\gamma_{1}, \gamma_{2}, \ldots, \gamma_{n}\right) & \text { if } t>0 \text { and } Q=0 \\ p_{t}\left(\gamma_{1}, \gamma_{2}, \ldots, \gamma_{n}\right)+Q^{-t} \beta & \text { if } t>0 \text { and } Q \neq 0\end{cases}
$$

when $\varphi=\left(x-\gamma_{1}\right)\left(x-\gamma_{2}\right) \ldots\left(x-\gamma_{n}\right)$. We see that the map (5.6.2) is injective, and it gives a bijection between $\mathbb{C}[x]_{\text {monic }}^{\langle Q\rangle} \times \mathbb{B}^{\langle Q\rangle}$ and the set of highest weight $\mathbf{u}=\left(u_{t}\right)_{\geq 0}$ satisfying (5.6.1), where we note that

$$
p_{t}(\gamma_{1}, \ldots, \gamma_{n}, \underbrace{Q^{-1}, \ldots, Q^{-1}}_{k})+Q^{-t} \beta=p_{t}\left(\gamma_{1}, \ldots, \gamma_{n}\right)+Q^{-t}(\beta+k) .
$$

Then we have the following corollary of Lemma 5.2 and Proposition 5.5.

Corollary 5.7. Any finite dimensional simple $U\left(\mathfrak{s l}_{2}^{\langle Q\rangle}[x]\right)$-module is isomorphic to $\mathcal{L}\left(\mathbf{u}^{\langle Q\rangle}(\varphi, \beta)\right)$ for some $(\varphi, \beta) \in \mathbb{C}[x]_{\text {monic }}^{\langle Q\rangle} \times \mathbb{B}^{\langle Q\rangle}$. Moreover, $\mathcal{L}\left(\mathbf{u}^{\langle Q\rangle}(\varphi, \beta)\right) \neq$ $\mathcal{L}\left(\mathbf{u}^{\langle Q\rangle}\left(\varphi^{\prime}, \beta^{\prime}\right)\right)$ if $(\varphi, \beta) \neq\left(\varphi^{\prime}, \beta^{\prime}\right)$.

5.8. Recall the evaluation modules from the paragraph 1.5 . Let $L(2)$ be the twodimensional simple $U\left(\mathfrak{s l}_{2}\right)$-module, and $v_{0} \in L(2)$ be a highest weight vector. We consider the evaluation module $L(2)^{\mathbf{e v}_{\gamma}}$ at $\gamma \in \mathbb{C}$, then we see that

$$
\mathcal{X}_{t}^{+} \cdot v_{0}=0, \quad \mathcal{J}_{t} \cdot v_{0}=\gamma^{t} v_{0} \quad(t \geq 0)
$$

in $L(2)^{\mathbf{e} \mathbf{v}_{\gamma}}$. 
For $\left(\varphi=\left(x-\gamma_{1}\right)\left(x-\gamma_{2}\right) \ldots\left(x-\gamma_{n}\right), \beta\right) \in \mathbb{C}[x]_{\text {monic }}^{\langle Q\rangle} \times \mathbb{B}^{\langle Q\rangle}$, we consider the $U\left(\mathfrak{s l}_{2}^{\langle Q\rangle}[x]\right)$-module

$$
\mathcal{N}_{(\varphi, \beta)}=L(2)^{\mathbf{e v}_{\gamma_{1}}} \otimes L(2)^{\mathbf{e v}_{\gamma_{2}}} \otimes \cdots \otimes L(2)^{\mathbf{e v}_{\gamma_{n}}} \otimes \mathcal{L}^{\beta}
$$

where $\mathcal{L}^{\beta}$ is the 1-dimensional $U\left(\mathfrak{s l}_{2}^{\langle Q\rangle}[x]\right)$-module given in the paragraph 5.1. Let $v_{0}^{(k)} \in L(2)^{\mathbf{e v}_{\gamma_{k}}}(1 \leq k \leq n)$ be a highest weight vector, and $\mathcal{L}^{\beta}=\mathbb{C} w_{0}$. Put $v_{(\varphi, \beta)}=v_{0}^{(1)} \otimes v_{0}^{(2)} \otimes \cdots \otimes v_{0}^{(n)} \otimes w_{0}$. Then, for $t \geq 0$, we have

$$
\mathcal{X}_{t}^{+} \cdot v_{(\varphi, \beta)}=0
$$

and

$$
\mathcal{J}_{t} \cdot v_{(\varphi, \beta)}= \begin{cases}(n+\beta) v_{(\varphi, \beta)} & \text { if } t=0, \\ p_{t}\left(\gamma_{1}, \gamma_{2}, \ldots, \gamma_{n}\right) v_{(\varphi, \beta)} & \text { if } t>0 \text { and } Q=0 \\ \left(p_{t}\left(\gamma_{1}, \gamma_{2}, \ldots, \gamma_{n}\right)+Q^{-t} \beta\right) v_{(\varphi, \beta)} & \text { if } t>0 \text { and } Q \neq 0\end{cases}
$$

Let $\mathcal{N}_{(\varphi, \beta)}^{\prime}$ be the $U\left(\mathfrak{s l}_{2}^{\langle Q\rangle}[x]\right)$-submodule of $\mathcal{N}_{(\varphi, \beta)}$ generated by $v_{(\varphi, \beta)}$. Then (5.8.1) and (5.8.2) imply that $\mathcal{N}_{(\varphi, \beta)}^{\prime}$ is a highest weight module of highest weight $\mathbf{u}^{\langle Q\rangle}(\varphi, \beta)$, and $\mathcal{N}_{(\varphi, \beta)}^{\prime} / \operatorname{rad} \mathcal{N}_{(\varphi, \beta)}^{\prime}$ is isomorphic to the simple highest weight module $\mathcal{L}\left(\mathbf{u}^{\langle Q\rangle}(\varphi, \beta)\right)$. From the construction, $\mathcal{L}\left(\mathbf{u}^{\langle Q\rangle}(\varphi, \beta)\right) \cong \mathcal{N}_{(\varphi, \beta)}^{\prime} / \operatorname{rad} \mathcal{N}_{(\varphi, \beta)}^{\prime}$ is finite dimensional for each $(\varphi, \beta) \in \mathbb{C}[x]_{\text {monic }}^{\langle Q\rangle} \times \mathbb{B}^{\langle Q\rangle}$. Combining with Corollary 5.7, we have the following classification of finite dimensional simple $U\left(\mathfrak{s l}_{2}^{\langle Q\rangle}[x]\right)$-modules.

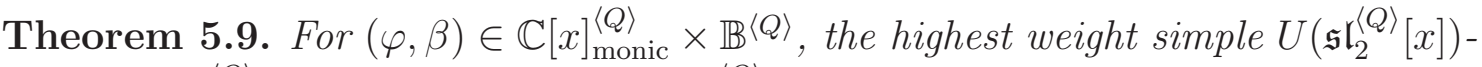
module $\mathcal{L}\left(\mathbf{u}^{\langle Q\rangle}(\varphi, \beta)\right)$ of highest weight $\mathbf{u}^{\langle Q\rangle}(\varphi, \beta)$ is finite dimensional, and we have that

$$
\mathcal{L}\left(\mathbf{u}^{\langle Q\rangle}(\varphi, \beta)\right) \cong \mathcal{L}\left(\mathbf{u}^{\langle Q\rangle}\left(\varphi^{\prime}, \beta^{\prime}\right)\right) \Leftrightarrow(\varphi, \beta)=\left(\varphi^{\prime}, \beta^{\prime}\right)
$$

for $(\varphi, \beta),\left(\varphi^{\prime}, \beta^{\prime}\right) \in \mathbb{C}[x]_{\text {monic }}^{\langle Q\rangle} \times \mathbb{B}^{\langle Q\rangle}$. Moreover

$$
\left\{\mathcal{L}\left(\mathbf{u}^{\langle Q\rangle}(\varphi, \beta)\right) \mid(\varphi, \beta) \in \mathbb{C}[x]_{\text {monic }}^{\langle Q\rangle} \times \mathbb{B}^{\langle Q\rangle}\right\}
$$

gives a complete set of isomorphism classes of finite dimensional simple $U\left(\mathfrak{s l}_{2}^{\langle Q\rangle}[x]\right)$ modules.

Remark 5.10. If $Q \neq 0$, the evaluation module $L(2)^{\mathbf{e v}_{Q^{-1}}}$ at $Q^{-1}$ is not simple. Recall that $L(2)$ is the two dimensional simple $U\left(\mathfrak{s l}_{2}\right)$-module with a highest weight vector $v_{0}$. Put $v_{1}=f \cdot v_{0}$. Then we see that $U\left(\mathfrak{s l}_{2}^{\langle Q\rangle}[x]\right) \cdot v_{1}=\mathbb{C} v_{1}$ is a proper $U\left(\mathfrak{s l}_{2}^{\langle Q\rangle}[x]\right)$-submodule of $L(2)^{\mathbf{e v}_{Q^{-1}}}$. Moreover, we have $L(2)^{\mathbf{e v}_{Q^{-1}}} / \mathbb{C} v_{1} \cong \mathcal{L}^{1}$ and $\mathbb{C} v_{1} \cong \mathcal{L}^{-1}$ as $U\left(\mathfrak{s} \mathfrak{r}_{2}^{\langle Q\rangle}[x]\right)$-modules. 


\section{$\S$ 6. Finite dimensional Simple $U\left(\mathfrak{s l}_{m}^{\langle\mathbf{Q}\rangle}[x]\right)$-Modules}

In this section, we classify the finite dimensional simple $U\left(\mathfrak{s l}_{m}^{\langle\mathbf{Q}\rangle}[x]\right)$-modules. By Proposition 2.6. any finite dimensional simple $U\left(\mathfrak{s}_{m}^{\langle\mathbf{Q}\rangle}[x]\right)$-module is isomorphic to the simple highest weight module $\mathcal{L}(\mathbf{u})$ of highest weight $\mathbf{u}=\left(u_{i, t}\right) \in \prod_{i=1}^{m-1} \prod_{t>0} \mathbb{C}$. Thus, it is enough to classify the highest weight $\mathbf{u}$ such that $\mathcal{L}(\mathbf{u})$ is finite dimensional.

6.1. 1-dimensional representations. First, we consider the 1-dimensional representations of $\mathfrak{s l}_{m}^{\langle Q\rangle}[x]$. For each $i=1,2, \ldots, m-1$, by checking the defining relations, we have the homomorphism of algebras

$$
\iota_{i}: U\left(\mathfrak{s l}_{2}^{\left\langle Q_{i}\right\rangle}[x]\right) \rightarrow U\left(\mathfrak{s l}_{m}^{\langle\mathbf{Q}\rangle}[x]\right) \text { by } \mathcal{X}_{t}^{ \pm} \mapsto \mathcal{X}_{i, t}^{ \pm}, \mathcal{J}_{t} \mapsto \mathcal{J}_{i, t} \quad(t \geq 0)
$$

Let $L=\mathbb{C} v$ be a 1-dimensional $U\left(\mathfrak{s l}_{m}^{\langle\mathbf{Q}\rangle}[x]\right)$-module. For each $i=1,2, \ldots, m-1$, when we regard $L$ as a $U\left(\mathfrak{s}_{2}^{\left\langle Q_{i}\right\rangle}[x]\right)$-module through the homomorphism $\iota_{i}$, we see that $L$ is isomorphic to $\mathcal{L}^{\beta_{i}}$ for some $\beta_{i} \in \mathbb{B}^{\left\langle Q_{i}\right\rangle}$ by Lemma 5.2. Thus, we have

$$
\mathcal{X}_{i, t}^{ \pm} \cdot v=0, \quad \mathcal{J}_{i, t} \cdot v=\left\{\begin{array}{ll}
0 & \text { if } Q_{i}=0, \\
Q_{i}^{-t} \beta_{i} v & \text { if } Q_{i} \neq 0
\end{array} \quad(1 \leq i \leq m-1, t \geq 0)\right.
$$

for some $\boldsymbol{\beta}=\left(\beta_{i}\right)_{1 \leq i \leq m-1} \in \prod_{i=1}^{m-1} \mathbb{B}^{\left\langle Q_{i}\right\rangle}$.

On the other hand, by checking the defining relations, we can define the 1dimensional $U\left(\mathfrak{s l}_{m}^{\langle\mathbf{Q}\rangle}[x]\right)$-module $\mathcal{L}^{\boldsymbol{\beta}}=\mathbb{C} v$ by (6.1.2) for each $\boldsymbol{\beta}=\left(\beta_{i}\right) \in \prod_{i=1}^{m-1} \mathbb{B}^{\left\langle Q_{i}\right\rangle}$. Now we proved the following lemma.

Lemma 6.2. Any 1-dimensional $U\left(\mathfrak{s l}_{m}^{\langle Q\rangle}\right)$-module is isomorphic to $\mathcal{L}^{\boldsymbol{\beta}}$ for some $\boldsymbol{\beta} \in \prod_{i=1}^{m-1} \mathbb{B}^{\left\langle Q_{i}\right\rangle}$.

6.3. For $\mathbf{u}=\left(u_{i, t}\right) \in \prod_{i=1}^{m-1} \prod_{t \geq 0} \mathbb{C}$, let $v_{0}$ be a highest weight vector of the simple highest weight $U\left(\mathfrak{s}_{m}^{\langle\mathbf{Q}\rangle}[x]\right)$-module $\mathcal{L}(\mathbf{u})$. When we regard $\mathcal{L}(\mathbf{u})$ as a $U\left(\mathfrak{s l}_{2}^{\left\langle Q_{i}\right\rangle}[x]\right)$ module through the homomorphism $\iota_{i}$ in (6.1.1) for each $i=1, \ldots, m-1$, we see that the $U\left(\mathfrak{s} \int_{2}^{\left\langle Q_{i}\right\rangle}[x]\right)$-submodule of $\mathcal{L}(\mathbf{u})$ generated by $v_{0}$ is a highest weight $U\left(\mathfrak{s i}{ }_{2}^{\left\langle Q_{i}\right\rangle}[x]\right)$ module of highest weight $\mathbf{u}_{i}=\left(u_{i, t}\right)_{t \geq 0} \in \prod_{t \geq 0} \mathbb{C}$ with the highest weight vector $v_{0}$. Then, if $\mathcal{L}(\mathbf{u})$ is finite dimensional, we see that $\mathbf{u}_{i}=\mathbf{u}^{\left\langle Q_{i}\right\rangle}\left(\varphi_{i}, \beta_{i}\right)$ for some $\left(\varphi_{i}, \beta_{i}\right) \in \mathbb{C}[x]_{\text {monic }}^{\left\langle Q_{i}\right\rangle} \times \mathbb{B}^{\left\langle Q_{i}\right\rangle}$ by Theorem 5.9 (or Corollary 5.7).

For $(\boldsymbol{\varphi}, \boldsymbol{\beta})=\left(\left(\varphi_{i}, \beta_{i}\right)\right)_{1 \leq i \leq m-1} \in \prod_{i=1}^{m-1}\left(\mathbb{C}[x]_{\text {monic }}^{\left\langle Q_{i}\right\rangle} \times \mathbb{B}^{\left\langle Q_{i}\right\rangle}\right)$, we define

$$
\mathbf{u}^{\langle\mathbf{Q}\rangle}(\boldsymbol{\varphi}, \boldsymbol{\beta})=\left(\mathbf{u}^{\langle\mathbf{Q}\rangle}(\boldsymbol{\varphi}, \boldsymbol{\beta})_{i, t}\right)_{1 \leq i \leq m-1, t \geq 0} \in \prod_{i=1}^{m-1} \prod_{t \geq 0} \mathbb{C}
$$


by

$$
\mathbf{u}^{\langle\mathbf{Q}\rangle}(\boldsymbol{\varphi}, \boldsymbol{\beta})_{i, t}= \begin{cases}\operatorname{deg} \varphi_{i}+\beta_{i} & \text { if } t=0 \\ p_{t}\left(\gamma_{i, 1}, \gamma_{i, 2}, \ldots, \gamma_{i, n_{i}}\right) & \text { if } t>0 \text { and } Q_{i}=0 \\ p_{t}\left(\gamma_{i, 1}, \gamma_{i, 2}, \ldots, \gamma_{i, n_{i}}\right)+Q_{i}^{-t} \beta_{i} & \text { if } t>0 \text { and } Q_{i} \neq 0\end{cases}
$$

when $\varphi_{i}=\left(x-\gamma_{i, 1}\right)\left(x-\gamma_{i, 2}\right) \ldots\left(x-\gamma_{i, n_{i}}\right)(1 \leq i \leq m-1)$. Then we have that

$$
\left(\mathbf{u}^{\langle\mathbf{Q}\rangle}(\boldsymbol{\varphi}, \boldsymbol{\beta})_{i, t}\right)_{t \geq 0}=\mathbf{u}^{\left\langle Q_{i}\right\rangle}\left(\varphi_{i}, \beta_{i}\right)
$$

for each $i=1,2, \ldots, m-1$. From the definition, we see that

$$
\mathbf{u}^{\langle\mathbf{Q}\rangle}(\boldsymbol{\varphi}, \boldsymbol{\beta})=\mathbf{u}^{\langle\mathbf{Q}\rangle}\left(\boldsymbol{\varphi}^{\prime}, \boldsymbol{\beta}^{\prime}\right) \Leftrightarrow(\boldsymbol{\varphi}, \boldsymbol{\beta})=\left(\varphi^{\prime}, \boldsymbol{\beta}^{\prime}\right)
$$

for $(\boldsymbol{\varphi}, \boldsymbol{\beta}),\left(\boldsymbol{\varphi}^{\prime}, \boldsymbol{\beta}^{\prime}\right) \in \prod_{i=1}^{m-1}\left(\mathbb{C}[x]_{\text {monic }}^{\left\langle Q_{i}\right\rangle} \times \mathbb{B}^{\left\langle Q_{i}\right\rangle}\right)$. By the above argument, any finite dimensional simple $U\left(\mathfrak{s}_{m}^{\langle\mathbf{Q}\rangle}[x]\right)$-module is isomorphic to $\mathcal{L}\left(\mathbf{u}^{\langle\mathbf{Q}\rangle}(\boldsymbol{\varphi}, \boldsymbol{\beta})\right)$ for some $(\boldsymbol{\varphi}, \boldsymbol{\beta}) \in \prod_{i=1}^{m-1}\left(\mathbb{C}[x]_{\text {monic }}^{\left\langle Q_{i}\right\rangle} \times \mathbb{B}^{\left\langle Q_{i}\right\rangle}\right)$.

On the other hand, for each $(\boldsymbol{\varphi}, \boldsymbol{\beta}) \in \prod_{i=1}^{m-1}\left(\mathbb{C}[x]_{\text {monic }}^{\left\langle Q_{i}\right\rangle} \times \mathbb{B}^{\left\langle Q_{i}\right\rangle}\right)$, we can construct a finite dimensional highest weight $U\left(\mathfrak{s l}_{m}^{\langle\mathbf{Q}\rangle}[x]\right)$-module of highest weight $\mathbf{u}^{\langle\mathbf{Q}\rangle}(\boldsymbol{\varphi}, \boldsymbol{\beta})$ as follows.

Let $\omega_{j}(1 \leq j \leq m-1)$ be the fundamental weight of $\mathfrak{s l}_{m}$, and $L\left(\omega_{j}\right)$ be the simple highest weight $U\left(\mathfrak{s l}_{m}\right)$-module of highest weight $\omega_{j}$. Let $v_{0} \in L\left(\omega_{j}\right)$ be a highest weight vector, then we have $e_{i} \cdot v_{0}=0$ and $H_{i} \cdot v_{0}=\delta_{i j} v_{0}(1 \leq i \leq m-1)$ by the definition. Recall that $L\left(\omega_{j}\right)^{\mathbf{e v}_{\gamma}}$ is the evaluation module of $L\left(\omega_{j}\right)$ at $\gamma \in \mathbb{C}$. From the definition, we see that

$$
\mathcal{X}_{i, t}^{+} \cdot v_{0}=0, \quad \mathcal{J}_{i, t} \cdot v_{0}=\delta_{i j} \gamma^{t} v_{0} \quad(1 \leq i \leq m-1, t \geq 0)
$$

in $L\left(\omega_{j}\right)^{\mathbf{e v} \gamma}$.

For $(\boldsymbol{\varphi}, \boldsymbol{\beta})=\left(\left(\varphi_{i}, \beta_{i}\right)\right)_{1 \leq i \leq m-1} \in \prod_{i=1}^{m-1}\left(\mathbb{C}[x]_{\text {monic }}^{\left\langle Q_{i}\right\rangle} \times \mathbb{B}^{\left\langle Q_{i}\right\rangle}\right)$, we consider the $U\left(\mathfrak{s l}_{m}^{\langle\mathbf{Q}\rangle}[x]\right)$-module

$$
\mathcal{N}_{(\boldsymbol{\varphi}, \boldsymbol{\beta})}=\left(\bigotimes_{j=1}^{m-1} \bigotimes_{k=1}^{n_{j}} L\left(\omega_{j}\right)^{\mathbf{e v}_{\gamma_{j, k}}}\right) \otimes \mathcal{L}^{\boldsymbol{\beta}}
$$

where $n_{j}$ and $\gamma_{j, k}\left(1 \leq k \leq n_{j}\right)$ are determined by $\varphi_{j}=\left(x-\gamma_{j, 1}\right)\left(x-\gamma_{j, 2}\right) \ldots(x-$ $\left.\gamma_{j, n_{j}}\right)$ for each $j=1,2, \ldots, m-1$, and $\boldsymbol{\beta}=\left(\beta_{i}\right)_{1 \leq i \leq m-1}$. Let $v_{0}^{(j, k)} \in L\left(\omega_{j}\right)^{\mathbf{e v}_{\gamma_{j, k}}}$ $\left(1 \leq j \leq m-1,1 \leq k \leq n_{j}\right)$ be a highest weight vector, and $\mathcal{L}^{\boldsymbol{\beta}}=\mathbb{C} w_{0}$. Put $v_{(\boldsymbol{\varphi}, \boldsymbol{\beta})}=\left(\otimes_{j=1}^{m-1} \otimes_{k=1}^{n_{j}} v_{0}^{(j, k)}\right) \otimes w_{0} \in \mathcal{N}_{(\boldsymbol{\varphi}, \boldsymbol{\beta})}$, then we have

$$
\mathcal{X}_{i, t}^{+} \cdot v_{(\boldsymbol{\varphi}, \boldsymbol{\beta})}=0, \quad \mathcal{J}_{i, t} \cdot v_{(\boldsymbol{\varphi}, \boldsymbol{\beta})}=\mathbf{u}^{\langle\mathbf{Q}\rangle}(\boldsymbol{\varphi}, \boldsymbol{\beta})_{i, t} v_{(\boldsymbol{\varphi}, \boldsymbol{\beta})} \quad(1 \leq i \leq m-1, t \geq 0)
$$


by (6.3.2). Let $\mathcal{N}_{(\boldsymbol{\varphi}, \boldsymbol{\beta})}^{\prime}$ be the $U\left(\mathfrak{s l}_{m}^{\langle\mathbf{Q}\rangle}[x]\right)$-submodule of $\mathcal{N}_{(\boldsymbol{\varphi}, \boldsymbol{\beta})}$ generated by $v_{(\boldsymbol{\varphi}, \boldsymbol{\beta})}$. Then (6.3.3) implies that $\mathcal{N}_{(\boldsymbol{\varphi}, \boldsymbol{\beta})}^{\prime}$ is a finite dimensional highest weight module of highest weight $\mathbf{u}^{\langle\mathbf{Q}\rangle}(\boldsymbol{\varphi}, \boldsymbol{\beta})$. Then we obtain the following classification of finite dimensional simple $U\left(\mathfrak{s i}_{m}^{\langle\mathbf{Q}\rangle}[x]\right)$-modules.

Theorem 6.4. For $(\boldsymbol{\varphi}, \boldsymbol{\beta}) \in \prod_{i=1}^{m-1}\left(\mathbb{C}[x]_{\text {monic }}^{\left\langle Q_{i}\right\rangle} \times \mathbb{B}^{\left\langle Q_{i}\right\rangle}\right)$, the highest weight simple $U\left(\mathfrak{s l}_{m}^{\langle\mathbf{Q}\rangle}[x]\right)$-module $\mathcal{L}\left(\mathbf{u}^{\langle\mathbf{Q}\rangle}(\boldsymbol{\varphi}, \boldsymbol{\beta})\right)$ of highest weight $\mathbf{u}^{\langle\mathbf{Q}\rangle}(\boldsymbol{\varphi}, \boldsymbol{\beta})$ is finite dimensional, and we have that

$$
\mathcal{L}\left(\mathbf{u}^{\langle\mathbf{Q}\rangle}(\boldsymbol{\varphi}, \boldsymbol{\beta})\right) \cong \mathcal{L}\left(\mathbf{u}^{\langle\mathbf{Q}\rangle}\left(\boldsymbol{\varphi}^{\prime}, \boldsymbol{\beta}^{\prime}\right)\right) \Leftrightarrow(\boldsymbol{\varphi}, \boldsymbol{\beta})=\left(\boldsymbol{\varphi}^{\prime}, \boldsymbol{\beta}^{\prime}\right)
$$

for $(\boldsymbol{\varphi}, \boldsymbol{\beta}),\left(\boldsymbol{\varphi}^{\prime}, \boldsymbol{\beta}^{\prime}\right) \in \prod_{i=1}^{m-1}\left(\mathbb{C}[x]_{\text {monic }}^{\left\langle Q_{i}\right\rangle} \times \mathbb{B}^{\left\langle Q_{i}\right\rangle}\right)$ Moreover

$$
\left\{\mathcal{L}\left(\mathbf{u}^{\langle\mathbf{Q}\rangle}(\boldsymbol{\varphi}, \boldsymbol{\beta})\right) \mid(\boldsymbol{\varphi}, \boldsymbol{\beta}) \in \prod_{i=1}^{m-1}\left(\mathbb{C}[x]_{\text {monic }}^{\left\langle Q_{i}\right\rangle} \times \mathbb{B}^{\left\langle Q_{i}\right\rangle}\right)\right\}
$$

gives a complete set of isomorphism classes of finite dimensional simple $U\left(\mathfrak{s l}_{m}^{\langle\mathbf{Q}\rangle}[x]\right)$ modules.

\section{$\S 7$. Finite dimensional simple $U\left(\mathfrak{g}_{m}^{\langle\mathbf{Q}\rangle}[x]\right)$-Modules}

In this section, we classify the finite dimensional simple $U\left(\mathfrak{g} \mathfrak{l}_{m}^{\langle\mathbf{Q}\rangle}[x]\right)$-modules. By Proposition 1.4 (iii), $\mathfrak{s l}_{m}^{\langle\mathbf{Q}\rangle}[x]$ is a Lie subalgebra of $\mathfrak{g l}_{m}^{\langle\mathbf{Q}\rangle}[x]$. The difference of representations of $\mathfrak{g l}_{m}^{\langle\mathbf{Q}\rangle}[x]$ from one of $\mathfrak{s l}_{m}^{\langle\mathbf{Q}\rangle}[x]$ is given by the family of 1-dimensional $U\left(\mathfrak{g l}_{m}^{\langle\mathbf{Q}\rangle}[x]\right)$-modules $\left\{\widetilde{\mathcal{L}}^{\mathbf{h}} \mid \mathbf{h} \in \prod_{t \geq 0} \mathbb{C}\right\}$. We remark that $\widetilde{\mathcal{L}}^{\mathbf{h}}\left(\mathbf{h} \in \prod_{t \geq 0} \mathbb{C}\right)$ is isomorphic to the trivial representation as a $U\left(\mathfrak{s l}_{m}^{\langle\mathbf{Q}\rangle}[x]\right)$-module when we restrict the action.

7.1. 1-dimensional representations. For $\boldsymbol{\beta}=\left(\beta_{i}\right)_{1 \leq i \leq m-1} \in \prod_{i=1}^{m-1} \mathbb{B}^{\left\langle Q_{i}\right\rangle}$, by checking the defining relations, we can define the 1-dimensional $U\left(\mathfrak{g l}_{m}^{\langle\mathbf{Q}\rangle}[x]\right)$-module $\widetilde{\mathcal{L}}^{\beta}=\mathbb{C} v$ by

$$
\begin{aligned}
& \mathcal{X}_{i, t}^{ \pm} \cdot v=0, \quad \mathcal{J}_{i, t} \cdot v=\left\{\begin{array}{ll}
0 & \text { if } Q_{i}=0, \\
Q_{i}^{-t} \beta_{i} v & \text { if } Q_{i} \neq 0
\end{array} \quad(1 \leq i \leq m-1, t \geq 0)\right. \\
& \mathcal{I}_{j, t} \cdot v=\left(\sum_{k=j}^{m-1} \mathcal{J}_{k, t}\right) \cdot v \quad(1 \leq j \leq m-1, t \geq 0), \quad \mathcal{I}_{m, t} \cdot v=0 \quad(t \geq 0) .
\end{aligned}
$$

Note that $\mathcal{J}_{j, t}=\mathcal{I}_{j, t}-\mathcal{I}_{j+1, t}$ in $U\left(\mathfrak{g l}_{m}^{\langle\mathbf{Q}\rangle}[x]\right)$, we see that $\widetilde{\mathcal{L}}^{\beta} \cong \mathcal{L}^{\beta}$ as $U\left(\mathfrak{s} \mathfrak{l}_{m}^{\langle\mathbf{Q}\rangle}[x]\right)$ modules when we restrict the action on $\widetilde{\mathcal{L}}^{\beta}$ to $U\left(\mathfrak{s l}_{m}^{\langle\mathbf{Q}\rangle}[x]\right)$ through the injective homomorphism $\Upsilon$ in the proposition 1.4 (iii). 
For $\mathbf{h}=\left(h_{t}\right)_{t \geq 0} \in \prod_{t \geq 0} \mathbb{C}$, we can also define the 1-dimensional $U\left(\mathfrak{g} \mathfrak{r}_{m}^{\langle\mathbf{Q}\rangle}[x]\right)$ module $\widetilde{\mathcal{L}^{\mathbf{h}}}=\mathbb{C} v$ by

$$
\mathcal{X}_{i, t}^{ \pm} \cdot v=0, \quad \mathcal{I}_{j, t} \cdot v=h_{t} v \quad(1 \leq i \leq m-1,1 \leq j \leq m, t \geq 0)
$$

We see that $\widetilde{\mathcal{L}}^{\mathbf{h}} \cong \mathcal{L}^{\mathbf{0}}$ as $U\left(\mathfrak{s l}_{m}^{\langle\mathbf{Q}\rangle}[x]\right)$-modules when we restrict the action on $\widetilde{\mathcal{L}}^{\mathbf{h}}$ to $U\left(\mathfrak{s l}_{m}^{\langle\mathbf{Q}\rangle}[x]\right)$ where $\mathbf{0}=(0)_{1 \leq i \leq m-1} \in \prod_{i=1}^{m-1} \mathbb{B}^{\left\langle Q_{i}\right\rangle}$ (i.e. $\mathcal{L}^{\mathbf{0}}$ is the trivial representation). Then we have the following classification of 1-dimensional $\left.U(\mathfrak{g l}\}_{m}^{\langle\mathbf{Q}\rangle}\right)$-modules.

Lemma 7.2. Any 1-dimensional $U\left(\mathfrak{g l}_{m}^{\langle\mathbf{Q}\rangle}[x]\right)$-module is isomorphic to $\widetilde{\mathcal{L}}^{\boldsymbol{\beta}} \otimes \widetilde{\mathcal{L}}^{\mathbf{h}}$ for some $\boldsymbol{\beta} \in \prod_{i=1}^{m-1} \mathbb{B}^{\left\langle Q_{i}\right\rangle}$ and $\mathbf{h} \in \prod_{t \geq 0} \mathbb{C}$. We have that

$$
\widetilde{\mathcal{L}}^{\boldsymbol{\beta}} \otimes \widetilde{\mathcal{L}}^{\mathbf{h}} \cong \widetilde{\mathcal{L}}^{\boldsymbol{\beta}^{\prime}} \otimes \widetilde{\mathcal{L}}^{\mathbf{h}^{\prime}} \Leftrightarrow(\boldsymbol{\beta}, \mathbf{h})=\left(\boldsymbol{\beta}^{\prime}, \mathbf{h}^{\prime}\right)
$$

Moreover, we see that $\widetilde{\mathcal{L}}^{\boldsymbol{\beta}} \otimes \widetilde{\mathcal{L}}^{\mathbf{h}} \cong \mathcal{L}^{\boldsymbol{\beta}}$ as $U\left(\mathfrak{s l}_{m}^{\langle\mathbf{Q}\rangle}[x]\right)$-modules when we restrict the action on $\widetilde{\mathcal{L}}^{\boldsymbol{\beta}} \otimes \widetilde{\mathcal{L}}^{\mathbf{h}}$ to $U\left(\mathfrak{s l}_{m}^{\langle\mathbf{Q}\rangle}[x]\right)$.

Proof. Let $\mathcal{L}=\mathbb{C} v$ be a 1 -dimensional $U\left(\mathfrak{g l}_{m}^{\langle\mathbf{Q}\rangle}\right)$-module. By restricting the action on $\mathcal{L}$ to $U\left(\mathfrak{s l}_{m}^{\langle\mathbf{Q}\rangle}\right)$ through the injective homomorphism $\Upsilon$ in the proposition 1.4 (iii), we have

$$
\begin{aligned}
& \mathcal{X}_{i, t}^{ \pm} \cdot v=0 \\
& \mathcal{J}_{i, t} \cdot v=\left(\mathcal{I}_{i, t}-\mathcal{I}_{i+1, t}\right) \cdot v=\left\{\begin{array}{ll}
0 & \text { if } Q_{i}=0 \\
Q_{i}^{-t} \beta_{i} v & \text { if } Q_{i} \neq 0
\end{array} \quad(1 \leq i \leq m-1, t \geq 0)\right.
\end{aligned}
$$

for some $\boldsymbol{\beta}=\left(\beta_{i}\right) \in \prod_{i=1}^{m-1} \mathbb{B}^{\left\langle Q_{i}\right\rangle}$ by Lemma 6.2.

On the other hand, for $t \in \mathbb{Z}_{\geq 0}$, there exists $h_{t} \in \mathbb{C}$ such that

$$
\mathcal{I}_{m, t} \cdot v=h_{t} v
$$

since $\operatorname{dim} \mathcal{L}=1$. Then (7.2.1) and (7.2.2) imply taht

$$
\mathcal{I}_{j, t} \cdot v=\left(\sum_{k=j}^{m-1} \mathcal{J}_{k, t}+h_{t}\right) \cdot v \quad(1 \leq j \leq m-1, t \geq 0), \quad \mathcal{I}_{m, t} \cdot v=h_{t} v \quad(t \geq 0)
$$

Then we see that $\mathcal{L} \cong \widetilde{\mathcal{L}}^{\boldsymbol{\beta}} \otimes \widetilde{\mathcal{L}}^{\mathbf{h}}$. The remaining statements are clear.

7.3. For $\widetilde{\mathbf{u}}=\left(\widetilde{u}_{j, t}\right) \in \prod_{j=1}^{m} \prod_{t \geq 0} \mathbb{C}$, let $v_{0}$ be a highest weight vector of the simple highest weight $U\left(\mathfrak{g} \mathfrak{r}_{m}^{\langle\mathbf{Q}\rangle}[x]\right)$-module $\mathcal{L}(\widetilde{\mathbf{u}})$. By restricting the action on $\mathcal{L}(\widetilde{\mathbf{u}})$ to $U\left(\mathfrak{s l}_{m}^{\langle\mathbf{Q}\rangle}[x]\right)$, Theorem 6.4 implies that

$$
\widetilde{u}_{i, t}-\widetilde{u}_{i+1, t}=\mathbf{u}^{\langle\mathbf{Q}\rangle}(\boldsymbol{\varphi}, \boldsymbol{\beta})_{i, t} \quad(1 \leq i \leq m-1, t \geq 0)
$$


for some $(\boldsymbol{\varphi}, \boldsymbol{\beta}) \in \prod_{i=1}^{m-1}\left(\mathbb{C}[x]_{\text {monic }}^{\left\langle Q_{i}\right\rangle} \times \mathbb{B}^{\left\langle Q_{i}\right\rangle}\right)$ if $\mathcal{L}(\widetilde{\mathbf{u}})$ is finite dimensional.

For $t \in \mathbb{Z}_{\geq 0}$, let $h_{t} \in \mathbb{C}$ be such that

$$
\widetilde{u}_{m, t}=h_{t} .
$$

By (7.3.1) and (7.3.2), we have

$$
\widetilde{u}_{j, t}=\sum_{k=j}^{m-1} \mathbf{u}^{\langle\mathbf{Q}\rangle}(\boldsymbol{\varphi}, \boldsymbol{\beta})_{k, t}+h_{t} \quad(1 \leq j \leq m-1, t \geq 0), \quad \widetilde{u}_{m, t}=h_{t} \quad(t \geq 0)
$$

for some $(\boldsymbol{\varphi}, \boldsymbol{\beta}) \in \prod_{i=1}^{m-1}\left(\mathbb{C}[x]_{\text {monic }}^{\left\langle Q_{i}\right\rangle} \times \mathbb{B}^{\left\langle Q_{i}\right\rangle}\right)$ and $\mathbf{h}=\left(h_{t}\right) \in \prod_{t \geq 0} \mathbb{C}$ if $\mathcal{L}(\widetilde{\mathbf{u}})$ is finite dimensional.

For $(\boldsymbol{\varphi}, \boldsymbol{\beta}, \mathbf{h})=\left(\left(\varphi_{i}, \beta_{i}\right)_{1 \leq i \leq m-1},\left(h_{t}\right)_{t \geq 0}\right) \in \prod_{i=1}^{m-1}\left(\mathbb{C}[x]_{\text {monic }}^{\left\langle Q_{i}\right\rangle} \times \mathbb{B}^{\left\langle Q_{i}\right\rangle}\right) \times \prod_{t \geq 0} \mathbb{C}$, we define

$$
\widetilde{\mathbf{u}}^{\langle\mathbf{Q}\rangle}(\boldsymbol{\varphi}, \boldsymbol{\beta}, \mathbf{h})=\left(\widetilde{\mathbf{u}}^{\langle\mathbf{Q}\rangle}(\boldsymbol{\varphi}, \boldsymbol{\beta}, \mathbf{h})_{j, t}\right) \in \prod_{j=1}^{m} \prod_{t \geq 0} \mathbb{C}
$$

by

$$
\widetilde{\mathbf{u}}^{\langle\mathbf{Q}\rangle}(\boldsymbol{\varphi}, \boldsymbol{\beta}, \mathbf{h})_{j, t}= \begin{cases}\sum_{k=j}^{m-1} \mathbf{u}^{\langle\mathbf{Q}\rangle}(\boldsymbol{\varphi}, \boldsymbol{\beta})_{k, t}+h_{t} & \text { if } 1 \leq j \leq m-1 \text { and } t \geq 0, \\ h_{t} & \text { if } j=m \text { and } t \geq 0 .\end{cases}
$$

From the definition, we see that

$$
\widetilde{\mathbf{u}}^{\langle\mathbf{Q}\rangle}(\boldsymbol{\varphi}, \boldsymbol{\beta}, \mathbf{h})=\widetilde{\mathbf{u}}^{\langle\mathbf{Q}\rangle}\left(\boldsymbol{\varphi}^{\prime}, \boldsymbol{\beta}^{\prime}, \mathbf{h}^{\prime}\right) \Leftrightarrow(\boldsymbol{\varphi}, \boldsymbol{\beta}, \mathbf{h})=\left(\boldsymbol{\varphi}^{\prime}, \boldsymbol{\beta}^{\prime}, \mathbf{h}^{\prime}\right)
$$

for $(\boldsymbol{\varphi}, \boldsymbol{\beta}, \mathbf{h}),\left(\boldsymbol{\varphi}^{\prime}, \boldsymbol{\beta}^{\prime}, \mathbf{h}^{\prime}\right) \in \prod_{i=1}^{m-1}\left(\mathbb{C}[x]_{\text {monic }}^{\left\langle Q_{i}\right\rangle} \times \mathbb{B}^{\left\langle Q_{i}\right\rangle}\right) \times \prod_{t \geq 0} \mathbb{C}$. By the above argument, any finite dimensional simple $U\left(\mathfrak{g l}_{m}^{\langle\mathbf{Q}\rangle}[x]\right)$-module is isomorphic to $\mathcal{L}\left(\widetilde{\mathbf{u}}^{\langle\mathbf{Q}\rangle}(\boldsymbol{\varphi}, \boldsymbol{\beta}, \mathbf{h})\right)$ for some $(\boldsymbol{\varphi}, \boldsymbol{\beta}, \mathbf{h}) \in \prod_{i=1}^{m-1}\left(\mathbb{C}[x]_{\text {monic }}^{\left\langle Q_{i}\right\rangle} \times \mathbb{B}^{\left\langle Q_{i}\right\rangle}\right) \times \prod_{t \geq 0} \mathbb{C}$.

On the other hand, for each $(\boldsymbol{\varphi}, \boldsymbol{\beta}, \mathbf{h}) \in \prod_{i=1}^{m-1}\left(\mathbb{C}[x]_{\text {monic }}^{\left\langle Q_{i}\right\rangle} \times \mathbb{B}^{\left\langle Q_{i}\right\rangle}\right) \times \prod_{t \geq 0} \mathbb{C}$, we can construct a finite dimensional highest weight $U\left(\mathfrak{g} \mathfrak{g}_{m}^{\langle\mathbf{Q}\rangle}[x]\right)$-module of highest weight $\widetilde{\mathbf{u}}^{\langle\mathbf{Q}\rangle}(\boldsymbol{\varphi}, \boldsymbol{\beta}, \mathbf{h})$ as follows.

Let $P=\bigoplus_{i=1}^{m} \mathbb{Z} \varepsilon_{i}$ be the weight lattice of $\mathfrak{g l}_{m}$. Put $\widetilde{\omega}_{l}=\varepsilon_{1}+\varepsilon_{2}+\cdots+\varepsilon_{l}$ for $l=1,2, \ldots, m-1$. Let $L\left(\widetilde{\omega}_{l}\right)$ be the simple highest weight $U\left(\mathfrak{g l}_{m}\right)$-module of highest weight $\widetilde{\omega}_{l}$, and $v_{0} \in L\left(\widetilde{\omega}_{l}\right)$ be a highest weight vector. Then, we have

$$
e_{i} \cdot v_{0}=0 \quad(1 \leq i \leq m-1) \text { and } K_{j} \cdot v_{0}= \begin{cases}v_{0} & \text { if } 1 \leq j \leq l \\ 0 & \text { if } l<j \leq m\end{cases}
$$


Recall that $L\left(\widetilde{\omega}_{l}\right)^{\widetilde{\operatorname{ev}}_{\gamma}}$ is the evaluation module of $L\left(\widetilde{\omega}_{l}\right)$ at $\gamma \in \mathbb{C}$. From the definition, we see that

$$
\mathcal{X}_{i, t}^{+} \cdot v_{0}=0 \quad(1 \leq i \leq m-1, t \geq 0), \quad \mathcal{I}_{j, t} \cdot v_{0}=\left\{\begin{array}{ll}
\gamma^{t} v_{0} & \text { if } 1 \leq j \leq l, \\
0 & \text { if } l<j \leq m
\end{array} \quad(t \geq 0)\right.
$$

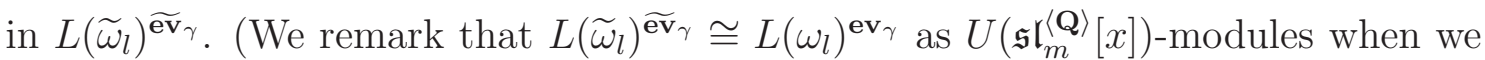
restrict the action on $L\left(\widetilde{\omega}_{l}\right)^{\widetilde{\operatorname{ev}}_{\gamma}}$ to $U\left(\mathfrak{s l}_{m}^{\langle\mathbf{Q}\rangle}[x]\right)$.)

For $(\boldsymbol{\varphi}, \boldsymbol{\beta}, \mathbf{h})=\left(\left(\varphi_{i}, \beta_{i}\right)_{1 \leq i \leq m-1},\left(h_{t}\right)_{t \geq 0}\right) \in \prod_{i=1}^{m-1}\left(\mathbb{C}[x]_{\text {monic }}^{\left\langle Q_{i}\right\rangle} \times \mathbb{B}^{\left\langle Q_{i}\right\rangle}\right) \times \prod_{t \geq 0} \mathbb{C}$, we consider the $U\left(\mathfrak{g l}_{m}^{\langle\mathbf{Q}\rangle}[x]\right)$-module

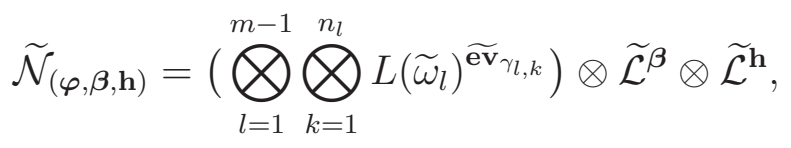

where $n_{l}$ and $\gamma_{l, k}\left(1 \leq k \leq n_{l}\right)$ are determined by $\varphi_{l}=\left(x-\gamma_{l, 1}\right)\left(x-\gamma_{l, 2}\right) \ldots\left(x-\gamma_{l, n_{l}}\right)$ for each $l=1,2, \ldots, m-1$, and we put $\boldsymbol{\beta}=\left(\beta_{i}\right)_{1 \leq i \leq m-1}$ and $\mathbf{h}=\left(h_{t}\right)_{t \geq 0}$. Let $v_{0}^{(l, k)} \in L\left(\widetilde{\omega}_{l}\right)^{\widetilde{\operatorname{ev}}_{\gamma_{l, k}}}\left(1 \leq l \leq m-1,1 \leq k \leq n_{l}\right)$ be a highest weight vector, $\widetilde{\mathcal{L}}^{\boldsymbol{\beta}}=\mathbb{C} w_{0}$ and $\widetilde{\mathcal{L}}^{\mathbf{h}}=\mathbb{C} z_{0}$. Put $v_{(\boldsymbol{\varphi}, \boldsymbol{\beta}, \mathbf{h})}=\left(\otimes_{l=1}^{m-1} \otimes_{k=1}^{n_{l}} v_{0}^{(l, k)}\right) \otimes w_{0} \otimes z_{0} \in \widetilde{\mathcal{N}}_{(\boldsymbol{\varphi}, \boldsymbol{\beta})}$, then we have

$$
\mathcal{X}_{i, t}^{+} \cdot v_{(\boldsymbol{\varphi}, \boldsymbol{\beta}, \mathbf{h})}=0, \quad \mathcal{I}_{j, t} \cdot v_{(\boldsymbol{\varphi}, \boldsymbol{\beta}, \mathbf{h})}=\widetilde{\mathbf{u}}^{\langle\mathbf{Q}\rangle}(\boldsymbol{\varphi}, \boldsymbol{\beta}, \mathbf{h})_{j, t} v_{(\boldsymbol{\varphi}, \boldsymbol{\beta}, \mathbf{h})}
$$

for $1 \leq i \leq m-1,1 \leq j \leq m$ and $t \geq 0$ by $(\overline{7.3 .3)})$. Let $\tilde{\mathcal{N}}_{(\boldsymbol{\varphi}, \boldsymbol{\beta}, \mathbf{h})}^{\prime}$ be the $U\left(\mathfrak{g l}_{m}^{\langle\mathbf{Q}\rangle}[x]\right)$ submodule of $\widetilde{\mathcal{N}}_{(\boldsymbol{\varphi}, \boldsymbol{\beta}, \mathbf{h})}$ generated by $v_{(\boldsymbol{\varphi}, \boldsymbol{\beta}, \mathbf{h})}$. Then (7.3.4) implies that $\tilde{\mathcal{N}}_{(\boldsymbol{\varphi}, \boldsymbol{\beta}, \mathbf{h})}^{\prime}$ is a finite dimensional highest weight module of highest weight $\widetilde{\mathbf{u}}^{\langle\mathbf{Q}\rangle}(\boldsymbol{\varphi}, \boldsymbol{\beta}, \mathbf{h})$. Now we obtain the following classification of finite dimensional simple $U\left(\mathfrak{g l} \mathfrak{g l}_{m}^{\langle\mathbf{Q}\rangle}[x]\right)$-modules.

Theorem 7.4. For $(\boldsymbol{\varphi}, \boldsymbol{\beta}, \mathbf{h}) \in \prod_{i=1}^{m-1}\left(\mathbb{C}[x]_{\text {monic }}^{\left\langle Q_{i}\right\rangle} \times \mathbb{B}^{\left\langle Q_{i}\right\rangle}\right) \times \prod_{t>0} \mathbb{C}$, the highest weight simple $U\left(\mathfrak{g l}_{m}^{\langle\mathbf{Q}\rangle}[x]\right)$-module $\mathcal{L}\left(\widetilde{\mathbf{u}}^{\langle\mathbf{Q}\rangle}(\boldsymbol{\varphi}, \boldsymbol{\beta}, \mathbf{h})\right)$ of highest weight $\widetilde{\mathbf{u}}^{\langle\mathbf{Q}\rangle}(\boldsymbol{\varphi}, \boldsymbol{\beta}, \mathbf{h})$ is finite dimensional, and we have that

$$
\mathcal{L}\left(\widetilde{\mathbf{u}}^{\langle\mathbf{Q}\rangle}(\boldsymbol{\varphi}, \boldsymbol{\beta}, \mathbf{h})\right) \cong \mathcal{L}\left(\widetilde{\mathbf{u}}^{\langle\mathbf{Q}\rangle}\left(\boldsymbol{\varphi}^{\prime}, \boldsymbol{\beta}^{\prime}, \mathbf{h}^{\prime}\right)\right) \Leftrightarrow(\boldsymbol{\varphi}, \boldsymbol{\beta}, \mathbf{h})=\left(\boldsymbol{\varphi}^{\prime}, \boldsymbol{\beta}^{\prime}, \mathbf{h}^{\prime}\right)
$$

for $(\boldsymbol{\varphi}, \boldsymbol{\beta}, \mathbf{h}),\left(\boldsymbol{\varphi}^{\prime}, \boldsymbol{\beta}^{\prime}, \mathbf{h}^{\prime}\right) \in \prod_{i=1}^{m-1}\left(\mathbb{C}[x]_{\text {monic }}^{\left\langle Q_{i}\right\rangle} \times \mathbb{B}^{\left\langle Q_{i}\right\rangle}\right) \times \prod_{t \geq 0} \mathbb{C}$. Moreover

$$
\left\{\mathcal{L}\left(\widetilde{\mathbf{u}}^{\langle\mathbf{Q}\rangle}(\boldsymbol{\varphi}, \boldsymbol{\beta}, \mathbf{h})\right) \mid(\boldsymbol{\varphi}, \boldsymbol{\beta}, \mathbf{h}) \in \prod_{i=1}^{m-1}\left(\mathbb{C}[x]_{\text {monic }}^{\left\langle Q_{i}\right\rangle} \times \mathbb{B}^{\left\langle Q_{i}\right\rangle}\right) \times \prod_{t \geq 0} \mathbb{C}\right\}
$$

gives a complete set of isomorphism classes of finite dimensional simple $U\left(\mathfrak{g} \mathfrak{r}_{m}^{\langle\mathbf{Q}\rangle}[x]\right)$ modules.

We also have the following corollary. 
Corollary 7.5. For $(\boldsymbol{\varphi}, \boldsymbol{\beta}, \mathbf{h}) \in \prod_{i=1}^{m-1}\left(\mathbb{C}[x]_{\text {monic }}^{\left\langle Q_{i}\right\rangle} \times \mathbb{B}^{\left\langle Q_{i}\right\rangle}\right) \times \prod_{t \geq 0} \mathbb{C}$, we have

$$
\mathcal{L}\left(\widetilde{\mathbf{u}}^{\langle\mathbf{Q}\rangle}(\boldsymbol{\varphi}, \boldsymbol{\beta}, \mathbf{h})\right) \cong \mathcal{L}\left(\mathbf{u}^{\langle\mathbf{Q}\rangle}(\boldsymbol{\varphi}, \boldsymbol{\beta})\right) \text { as } U\left(\mathfrak{s l}_{m}^{\langle\mathbf{Q}\rangle}[x]\right) \text {-modules }
$$

when we restrict the action on $\mathcal{L}\left(\widetilde{\mathbf{u}}^{\langle\mathbf{Q}\rangle}(\boldsymbol{\varphi}, \boldsymbol{\beta}, \mathbf{h})\right)$ to $U\left(\mathfrak{s l}_{m}^{\langle\mathbf{Q}\rangle}[x]\right)$.

Proof. We prove that $\mathcal{L}\left(\widetilde{\mathbf{u}}^{\langle\mathbf{Q}\rangle}(\boldsymbol{\varphi}, \boldsymbol{\beta}, \mathbf{h})\right)$ is also simple when we restrict the action to $U\left(\mathfrak{s l}_{m}^{\langle\mathbf{Q}\rangle}[x]\right)$. Then the isomorphism follows from the definitions of $\widetilde{\mathbf{u}}^{\langle\mathbf{Q}\rangle}(\boldsymbol{\varphi}, \boldsymbol{\beta}, \mathbf{h})$ and $\mathbf{u}^{\langle\mathbf{Q}\rangle}(\boldsymbol{\varphi}, \boldsymbol{\beta})$.

Let $v_{0} \in \mathcal{L}\left(\widetilde{\mathbf{u}}^{\langle\mathbf{Q}\rangle}(\boldsymbol{\varphi}, \boldsymbol{\beta}, \mathbf{h})\right)$ be a highest weight vector as the $U\left(\mathfrak{g l}_{m}^{\langle\mathbf{Q}\rangle}[x]\right)$-module. Then we have

$$
\mathcal{L}\left(\widetilde{\mathbf{u}}^{\langle\mathbf{Q}\rangle}(\boldsymbol{\varphi}, \boldsymbol{\beta}, \mathbf{h})\right)=U\left(\mathfrak{n}^{-}\right) \cdot v_{0}
$$

by the triangular decomposition in Proposition 1.4 (iv). This implies that

$$
\mathcal{L}\left(\widetilde{\mathbf{u}}^{\langle\mathbf{Q}\rangle}(\boldsymbol{\varphi}, \boldsymbol{\beta}, \mathbf{h})\right)=U\left(\mathfrak{s l}_{m}^{\langle\mathbf{Q}\rangle}[x]\right) \cdot v_{0}
$$

Assume that $\mathcal{L}\left(\widetilde{\mathbf{u}}^{\langle\mathbf{Q}\rangle}(\boldsymbol{\varphi}, \boldsymbol{\beta}, \mathbf{h})\right)$ is not simple as a $U\left(\mathfrak{s l}_{m}^{\langle\mathbf{Q}\rangle}[x]\right)$-module by the restriction, then $\mathcal{L}\left(\widetilde{\mathbf{u}}^{\langle\mathbf{Q}\rangle}(\boldsymbol{\varphi}, \boldsymbol{\beta}, \mathbf{h})\right)$ contains a non-zero proper simple $U\left(\mathfrak{s l}_{m}^{\langle\mathbf{Q}\rangle}[x]\right)$ submodule which is a highest weight $U\left(\mathfrak{s l}_{m}^{\langle\mathbf{Q}\rangle}[x]\right)$-module. This implies that there exist an element $w_{0} \in \mathcal{L}\left(\widetilde{\mathbf{u}}^{\langle\mathbf{Q}\rangle}(\boldsymbol{\varphi}, \boldsymbol{\beta}, \mathbf{h})\right)$ such that $\mathcal{X}_{i, t}^{+} \cdot w_{0}=0(1 \leq i \leq m-1, t \geq 0)$ and $w_{0} \notin \mathbb{C} v_{0}$. Then $U\left(\mathfrak{g l} \mathfrak{r}_{m}^{\langle\mathbf{Q}\rangle}[x]\right) \cdot w_{0}$ turns out to be a non-zero proper $U\left(\mathfrak{g} \mathfrak{l}_{m}^{\langle\mathbf{Q}\rangle}[x]\right)$ submodule of $\mathcal{L}\left(\widetilde{\mathbf{u}}^{\langle\mathbf{Q}\rangle}(\boldsymbol{\varphi}, \boldsymbol{\beta}, \mathbf{h})\right)$. This is a contradiction.

\section{Appendix A. Some COMbinatorics}

A.1. Let $\mathbb{Z}\left[x_{1}, \ldots, x_{n}\right]$ be the ring of polynomials in independent variables $x_{1}, \ldots, x_{n}$ over $\mathbb{Z}$. For $k \in \mathbb{Z}_{>0}$, put

$$
\begin{aligned}
& p_{k}\left(x_{1}, \ldots, x_{n}\right)=x_{1}^{k}+x_{2}^{k}+\cdots+x_{n}^{k} \in \mathbb{Z}\left[x_{1}, \ldots, x_{n}\right], \\
& e_{k}\left(x_{1}, \ldots, x_{n}\right)=\sum_{1 \leq i_{1}<i_{2}<\cdots<i_{k} \leq n} x_{i_{1}} x_{i_{2}} \ldots x_{i_{k}} \in \mathbb{Z}\left[x_{1}, \ldots, x_{n}\right] .
\end{aligned}
$$

Namely, $p_{k}\left(x_{1}, \ldots, x_{n}\right)$ is the power sum symmetric polynomial of degree $k$, and $e_{k}\left(x_{1}, \ldots, x_{n}\right)$ is the elementary symmetric polynomial of degree $k$. We also put $e_{0}\left(x_{1}, \ldots, x_{n}\right)=1$. Then, for $k>0$, we have

$$
k e_{k}\left(x_{1}, \ldots, x_{n}\right)=\sum_{z=1}^{k}(-1)^{z-1} p_{z}\left(x_{1}, \ldots, x_{n}\right) e_{k-z}\left(x_{1}, \ldots, x_{n}\right)
$$

by $\left[\mathrm{M}, \S 1\left(2.11^{\prime}\right)\right]$. For $s>n$, we have

$$
0=\sum_{z=1}^{s}(-1)^{z-1} p_{z}\left(x_{1}, \ldots, x_{n}\right) e_{s-z}\left(x_{1}, \ldots, x_{n}\right)
$$




$$
\begin{aligned}
& =\sum_{z=1}^{s-1}(-1)^{z-1} p_{z}\left(x_{1}, \ldots, x_{n}\right) e_{s-z}\left(x_{1}, \ldots, x_{n}\right)+(-1)^{s-1} p_{s}\left(x_{1}, \ldots, x_{n}\right) \\
& =\sum_{z=s-n}^{s-1}(-1)^{z-1} p_{z}\left(x_{1}, \ldots, x_{n}\right) e_{s-z}\left(x_{1}, \ldots, x_{n}\right)+(-1)^{s-1} p_{s}\left(x_{1}, \ldots, x_{n}\right),
\end{aligned}
$$

where we note that $e_{s-z}\left(x_{1}, \ldots, x_{n}\right)=0$ if $z<s-n$. Put $w=z-s+n$, we have

$$
\sum_{w=0}^{n-1}(-1)^{n-w+1} p_{s-n+w}\left(x_{1}, \ldots, x_{n}\right) e_{n-w}\left(x_{1}, \ldots, x_{n}\right)=p_{s}\left(x_{1}, \ldots, x_{n}\right)
$$

for $s>n$.

Lemma A.2. For $n \in \mathbb{Z}_{>0}$ and $u_{1}, u_{2}, \ldots, u_{n} \in \mathbb{C}$, the simultaneous equations

$$
\left\{\begin{array}{c}
p_{1}\left(x_{1}, x_{2}, \ldots, x_{n}\right)=u_{1} \\
p_{2}\left(x_{1}, x_{2}, \ldots, x_{n}\right)=u_{2}, \\
\vdots \\
p_{n}\left(x_{1}, x_{2}, \ldots, x_{n}\right)=u_{n}
\end{array}\right.
$$

has a solution in $\mathbb{C}$.

Proof. We prove the lemma by the induction on $n$. In the case where $n=1$, it is clear. If $n>1$, the equations (A.2.1) are equivalent to the equations

$$
\left\{\begin{array}{l}
p_{1}\left(x_{1}, x_{2}, \ldots, x_{n-1}\right)=u_{1}-x_{n} \\
p_{2}\left(x_{1}, x_{2}, \ldots, x_{n-1}\right)=u_{2}-x_{n}^{2} \\
\quad \vdots \\
p_{n-1}\left(x_{1}, x_{2}, \ldots, x_{n-1}\right)=u_{n-1}-x_{n}^{n-1} \\
p_{n}\left(x_{1}, x_{2}, \ldots, x_{n-1}\right)=u_{n}-x_{n}^{n} .
\end{array}\right.
$$

By (A.1.1), we have

$$
\begin{aligned}
& p_{n}\left(x_{1}, x_{2}, \ldots, x_{n-1}\right) \\
& =\sum_{i=1}^{n-1}(-1)^{i+n-1} p_{i}\left(x_{1}, x_{2}, \ldots, x_{n-1}\right) e_{n-i}\left(x_{1}, x_{2}, \ldots, x_{n-1}\right),
\end{aligned}
$$

where we note that $e_{n}\left(x_{1}, x_{2}, \ldots, x_{n-1}\right)=0$. On the other hand, we can write

$$
e_{n-i}\left(x_{1}, x_{2}, \ldots, x_{n-1}\right)=\sum_{\lambda \vdash n-i} \alpha_{\lambda} p_{\lambda}\left(x_{1}, x_{2}, \ldots, x_{n-1}\right)
$$


for some $\alpha_{\lambda} \in \mathbb{C}$, where $p_{\lambda}\left(x_{1}, x_{2}, \ldots, x_{n-1}\right)=\prod_{j=1}^{\ell(\lambda)} p_{\lambda_{j}}\left(x_{1}, x_{2}, \ldots, x_{n-1}\right)$ for $\lambda=$ $\left(\lambda_{1}, \lambda_{2}, \ldots\right) \vdash n-i$. Thus we have

$$
p_{n}\left(x_{1}, x_{2}, \ldots, x_{n-1}\right)=\sum_{i=1}^{n-1} \sum_{\lambda \vdash n-i}(-1)^{i+n-1} \alpha_{\lambda} p_{i}\left(x_{1}, x_{2}, \ldots, x_{n-1}\right) p_{\lambda}\left(x_{1}, x_{2}, \ldots, x_{n-1}\right)
$$

(Note that $\left\{p_{\mu}\left(x_{1}, x_{2}, \ldots, x_{n-1}\right) \mid \mu \vdash k\right\}$ is not linearly independent if $k \geq n$. For an example, we have $p_{(3)}\left(x_{1}, x_{2}\right)=\frac{3}{2} p_{(2,1)}\left(x_{1}, x_{2}\right)-\frac{1}{2} p_{(1,1,1)}\left(x_{1}, x_{2}\right)$.)

Then the equations (A.2.2) are equivalent to the equations

$$
\left\{\begin{array}{l}
p_{1}\left(x_{1}, x_{2}, \ldots, x_{n-1}\right)=u_{1}-x_{n}, \\
p_{2}\left(x_{1}, x_{2}, \ldots, x_{n-1}\right)=u_{2}-x_{n}^{2}, \\
\quad \vdots \\
p_{n-1}\left(x_{1}, x_{2}, \ldots, x_{n-1}\right)=u_{n-1}-x_{n}^{n-1}, \\
\sum_{i=1}^{n-1} \sum_{\lambda \vdash n-i}(-1)^{i+n-1} \alpha_{\lambda}\left(u_{i}-x_{n}^{i}\right) \prod_{j=1}^{\ell(\lambda)}\left(u_{\lambda_{j}}-x_{n}^{\lambda_{j}}\right)=u_{n}-x_{n}^{n} \quad \cdots(* 1) .
\end{array}\right.
$$

Let $\beta_{n}$ be a solution of the equation $(* 1)$ for the variable $x_{n}$. By the assumption of the induction, the simultaneous equations

$$
\left\{\begin{array}{l}
p_{1}\left(x_{1}, x_{2}, \ldots, x_{n-1}\right)=u_{1}-\beta_{n} \\
p_{2}\left(x_{1}, x_{2}, \ldots, x_{n-1}\right)=u_{2}-\beta_{n}^{2} \\
\quad \vdots \\
p_{n-1}\left(x_{1}, x_{2}, \ldots, x_{n-1}\right)=u_{n-1}-\beta_{n}^{n-1}
\end{array}\right.
$$

for variables $x_{1}, x_{2}, \ldots, x_{n-1}$ has a solution. We denote it by $\left(x_{1}, x_{2}, \ldots, x_{n-1}\right)=$ $\left(\beta_{1}, \beta_{2}, \ldots, \beta_{n-1}\right)$. Then $\left(x_{1}, x_{2}, \ldots, x_{n}\right)=\left(\beta_{1}, \beta_{2}, \ldots, \beta_{n}\right)$ gives a solution of (A.2.3).

A.3. We consider some modifications of the formulas (A.1.1) and A.1.2) as follows. Let $\mathbf{b}=\left(b_{1}, \ldots, b_{n}\right)$ be $n$ independent variables, and we consider the ring of polynomials $\mathbb{Z}\left[x_{1}, \ldots, x_{n}\right]\left[b_{1}, \ldots, b_{n}\right]$. For $k \in \mathbb{Z}_{>0}$, put

$$
\begin{aligned}
& e_{k}^{(\mathbf{b})}\left(x_{1}, \ldots, x_{n}\right) \\
& =\sum_{1 \leq i_{1}<i_{2}<\cdots<i_{k} \leq n}\left(b_{i_{1}}+b_{i_{2}}+\cdots+b_{i_{k}}\right) x_{i_{1}} x_{i_{2}} \ldots x_{i_{k}} \in \mathbb{Z}\left[x_{1}, \ldots, x_{n}\right]\left[b_{1}, \ldots, b_{n}\right]
\end{aligned}
$$

and

$$
p_{k}^{(\mathbf{b})}\left(x_{1}, \ldots, x_{n}\right)=b_{1} x_{1}^{k}+b_{2} x_{2}^{k}+\cdots+b_{n} x_{n}^{k} \in \mathbb{Z}\left[x_{1}, \ldots, x_{n}\right]\left[b_{1}, \ldots, b_{n}\right] .
$$


We also put $e_{0}^{(\mathbf{b})}=1$. Note that $e_{k}^{(\mathbf{b})}\left(x_{1}, \ldots, x_{n}\right)=0$ if $k>n$. Put $\mathbf{1}=(1,1, \ldots, 1)$, then we have $e_{k}^{(\mathbf{1})}\left(x_{1}, \ldots, x_{n}\right)=k e_{k}\left(x_{1}, \ldots, x_{n}\right)$ and $p_{k}^{(\mathbf{1})}\left(x_{1}, \ldots, x_{n}\right)=p_{k}\left(x_{1}, \ldots, x_{n}\right)$.

We consider the generating functions $E(t), E^{(\mathbf{b})}(t)$ and $P^{(\mathbf{b})}(t)$ by

$$
\begin{aligned}
& E(t)=\sum_{k \geq 0} e_{k}\left(x_{1}, \ldots, x_{n}\right) t^{k} \in \mathbb{Z}\left[x_{1}, \ldots, x_{n}\right]\left[b_{1}, \ldots, b_{n}\right][[t]], \\
& E^{(\mathbf{b})}(t)=\sum_{k \geq 0} e_{k+1}^{(\mathbf{b})}\left(x_{1}, \ldots, x_{n}\right) t^{k} \in \mathbb{Z}\left[x_{1}, \ldots, x_{n}\right]\left[b_{1}, \ldots, b_{n}\right][[t]], \\
& P^{(\mathbf{b})}(t)=\sum_{k \geq 0}(-1)^{k} p_{k+1}^{(\mathbf{b})}\left(x_{1}, \ldots, x_{n}\right) t^{k} \in \mathbb{Z}\left[x_{1}, \ldots, x_{n}\right]\left[b_{1}, \ldots, b_{n}\right][[t]] .
\end{aligned}
$$

Then, we have

$$
E(t)=\prod_{i=1}^{n}\left(1+x_{i} t\right), \quad P^{(\mathbf{b})}(t)=\sum_{i=1}^{n} \frac{b_{i} x_{i}}{1+x_{i} t}
$$

and

$$
P^{(\mathbf{b})}(t) E(t)=\sum_{i=1}^{n} b_{i} x_{i}\left(\prod_{j=1, j \neq i}^{n}\left(1+x_{i} t\right)\right)=E^{(\mathbf{b})}(t)
$$

This implies that, for $k \geq 0$,

$$
e_{k+1}^{(\mathbf{b})}\left(x_{1}, \ldots, x_{n}\right)=\sum_{z=0}^{k}(-1)^{z} p_{z+1}^{(\mathbf{b})}\left(x_{1}, \ldots, x_{n}\right) e_{k-z}\left(x_{1}, \ldots, x_{n}\right) .
$$

In the case where $k=n$, we have

$$
\sum_{z=0}^{n}(-1)^{z} p_{z+1}^{(\mathbf{b})}\left(x_{1}, \ldots, x_{n}\right) e_{n-z}\left(x_{1}, \ldots, x_{n}\right)=0
$$

since $e_{n+1}^{(\mathbf{b})}\left(x_{1}, \ldots, x_{n}\right)=0$. This implies that

$$
\sum_{z=0}^{n-1}(-1)^{n-z+1} p_{z+1}^{(\mathbf{b})}\left(x_{1}, \ldots, x_{n}\right) e_{n-z}\left(x_{1}, \ldots, x_{n}\right)=p_{n+1}^{(\mathbf{b})}\left(x_{1}, \ldots, x_{n}\right) .
$$

\section{REFERENCES}

[C] V. Chari, Integrable representations of affine Lie algebras, Invent. Math. 85 (1986), 317335.

[CP] V. Chari and A. Pressley, New unitary representations of loop groups, Math. Ann. 275 (1986), 87-104.

[M] I.G. Macdonald, Symmetric Functions and Hall Polynomials, 2nd edition, Oxford Univ. Press, 1995. 
[W] K. Wada, New Realization of Cyclotomic q-Schur Algebras, Publ. RIMS Kyoto Univ. 52 (2016), 497-555.

Department of Mathematics, Faculty of Science, Shinshu University, Asahi 31-1, Matsumoto 390-8621, Japan

E-mail address: wada@math.shinshu-u.ac.jp 